Zeros de polinômios característicos e estabilidade de métodos numéricos 
SERVIÇO DE PÓS-GRADUAÇÃO DO ICMC-USP

Data de Depósito: 15 de fevereiro de 2008

Assinatura:

\section{Zeros de polinômios característicos e estabilidade de métodos numéricos}

Vanessa Avansini Botta

Orientador: José Alberto Cuminato

Tese apresentada ao Instituto de Ciências Matemáticas e de Computação - ICMC/USP, como parte dos requisitos para obtenção do título de Doutor em Ciências de Computação e Matemática Computacional.

USP - São Carlos

Fevereiro/2008 
O decorrer deste trabalho, em meio a tantas descobertas, muitas dificuldades surgiram e também muitas alegrias floresceram. E nestes momentos inesquecíveis, a colaboração de algumas pessoas incomparáveis foi de extrema importância para a conclusão desta tese, que representa uma grande conquista.

Então, agradeço sinceramente:

À Deus, por tudo.

Aos meus familiares e amigos, que sempre me acolheram nos momentos mais difíceis.

Ao meu marido, Fabio, pela paciência e amor que sempre me dedicou.

A todos os professores que sempre me ajudaram, em especial ao Prof. Dr. Dimitar Kolev Dimitrov, pela disposição nos momentos em que precisei.

Ao Prof. Dr. José Alberto Cuminato, pela orientação deste trabalho, pela confiança e atenção.

Ao Prof. Dr. Messias Meneguette Junior, em especial, pela orientação deste trabalho, pela atenção, dedicação e paciência.

A todos que de alguma forma contribuíram para a realização deste trabalho. 
Teoria das equações diferenciais faz parte de uma área da Mate-
mática muito rica em aplicações. Os métodos numéricos para
a solução de equações diferenciais ordinárias são, da mesma forma que as próprias equações, fontes importantes de problemas a serem pesquisados. Como destaque tem-se os métodos multiderivadas de passo múltiplo, que são importantes na solução de problemas stiff. Os métodos numéricos mais conhecidos para a solução desses problemas são os BDF, que compõem, para $L=1$, a família dos métodos $(K, L)$ de Brown. Algumas questões relacionadas à estabilidade dos métodos $(K, L)$ ainda não foram solucionadas como, por exemplo, uma conjectura de Jeltsch. Para analisá-la, é necessário estudar o comportamento dos zeros dos polinômios característicos associados aos métodos $(K, L)$. Neste trabalho é apresentado um estudo sobre zeros de polinômios com o objetivo de demonstrar a validade da conjectura de Jeltsch para $K \leq K_{L}^{*}$. As regiões de estabilidade para alguns valores de $K$ e $L$ fixos são apresentadas e também é utilizada a teoria das order stars para mostrar algumas propriedades dos métodos $(K, L)$. Portanto, este trabalho apresenta um estudo sobre os métodos $(K, L)$ de Brown e usa uma ferramenta pouco utilizada na literatura, que são as order stars, para demonstrar alguns resultados.

Palavras-chave: métodos $(K, L)$ de Brown, estabilidade de métodos numéricos, zeros de polinômios característicos, order stars. 
He theory of differential equations is part of one area of Mathematics very rich in applications. The numerical methods for the solutions of ordinary differential equations are, in the same way as the equations themselves, important sources of problems to be studied. As prominence one has the multiderivative multistep methods which are important for the solution of stiff problems. The best known numerical methods for the solutions of these kind of problems are the BDF methods, which is part of the family of the Brown $(K, L)$ methods with $L=1$. Some questions about stability of the $(K, L)$ methods has not been solved yet as, for example, a conjecture by Jeltsch. In order to tackle this open problem, it becomes necessary to study the behavior of the zeros of the characteristic polynomials associated to the $(K, L)$ methods. In this work a study of the zeros of the characteristic polynomial is carried out aiming at proving Jeltsch conjecture for $K \leq K_{L}^{*}$. Regions of stability is shown for some fixed values of $K$ and $L$, as well as the use of order stars techniques are applied to show some properties of $(K, L)$ methods. Therefore, this work presents a study of Brown's $(K, L)$ methods, that makes use of a tool that seems not to have been used very often in the literature, the order stars, in order to prove the main results.

Key words: Brown $(K, L)$ methods, stability of numerical methods, zeros of characteristic polynomials, order stars. 
$\begin{array}{ll}\text { Agradecimentos } & \text { i }\end{array}$

Resumo ii

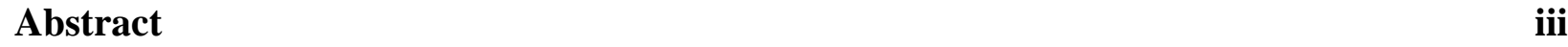

1 Introdução $\quad 1$

2 Zeros de Polinômios $\quad 11$

2.1 Resultados clássicos sobre zeros de polinômios . . . . . . . . . . . . . 11

2.1 .1 Localização dos zeros . . . . . . . . . . . . . . . . . 11

2.2 Análise da Conjectura $1.2 \ldots \ldots \ldots \ldots \ldots$

2.2.1 Limitante para os zeros . . . . . . . . . . . . 15

2.2.2 Polinômio reflexivo . . . . . . . . . . . . . . . . 15

2.2 .3 Caso geral . . . . . . . . . . . . . . . . 20

2.2.4 Condições adicionais . . . . . . . . . . . . . . . . . . 24

3 Métodos $(K, L)$ de Brown $\quad 30$

3.1 Introdução . . . . . . . . . . . . . . . . . . 30

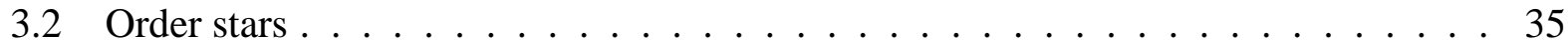

3.2 .1 Conceito geral . . . . . . . . . . . . . . 38

3.2.2 Order stars para os métodos de passo múltiplo . . . . . . . . . . . . 41

3.3 Propriedades dos métodos de Brown e order stars . . . . . . . . . . . . . . . . 43 
3.3.1 Order stars para os métodos $(K, L) \ldots \ldots \ldots \ldots$. . . . . . . 44

3.3.2 Comportamento dos zeros dos polinômios característicos . . . . . . . . 48

3.3.3 Resultados sobre zero-estabilidade . . . . . . . . . . . . . . 49

3.3.4 Regiões de estabilidade . . . . . . . . . . . . . . . . . . . 51

4 Considerações Finais $\quad 58$

4.1 Sobre zeros de polinômios . . . . . . . . . . . . . 58

4.2 Sobre os métodos $(K, L)$ de Brown $\ldots \ldots \ldots \ldots$. . . . . . . . 60

$\begin{array}{ll}\text { Referências Bibliográficas } & 63\end{array}$ 
1.1 Soluções $u(x)$ e $v(x) \ldots \ldots \ldots \ldots \ldots \ldots$

2.1 Comportamento dos zeros de $P_{\gamma}(z)=(1+\gamma) z^{3}+3 z^{2}+3 z+1,0 \leq \gamma \leq 1.2$. . 18

2.2 Comportamento dos zeros de $P_{\gamma}(z)=(1+\gamma) z^{4}+2 z^{3}+2 z^{2}+2 z+1,0 \leq \gamma \leq 1$. 19

2.3 Comportamento dos zeros de $P_{\gamma}(z)=(2.2+\gamma) z^{3}+6 z^{2}+6 z+2.2,0 \leq \gamma \leq 2$. . 19

2.4 Comportamento dos zeros de $P_{\gamma}(z)=(1+\gamma) z^{3}+2 z^{2}+2 z+1,0 \leq \gamma \leq 1$. . . 20

2.5 Comportamento dos zeros de $P_{\gamma}(z)=(1.5+\gamma) z^{3}+2 z^{2}+2 z+1.5,0 \leq \gamma \leq 1$. . 20

3.1 Order stars da aproximação de Padé $\ldots \ldots$. . . . . . . . . . . . . 38

3.2 Order star do BDF de 2 passos . . . . . . . . . . . . . . . . 44

3.3 Order star do BDF de 3 passos $\ldots \ldots \ldots \ldots \ldots \ldots$

3.4 Order star do BDF de 4 passos . . . . . . . . . . . . . . . . 44

3.5 Order star do BDF de 5 passos . . . . . . . . . . . . . . 45

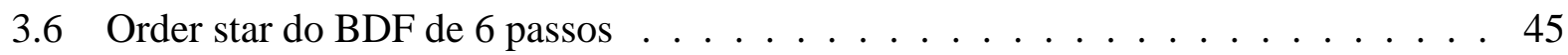

3.7 Order star do BDF de 7 passos . . . . . . . . . . . . . . 45

3.8 Order star do BDF de 8 passos $\ldots \ldots \ldots \ldots \ldots$

3.9 Order star do BDF de 9 passos . . . . . . . . . . . . . . . 45

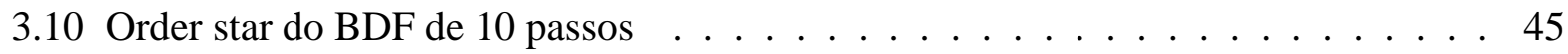

3.11 Order star do método $(3,2)$ de Brown $\ldots \ldots \ldots \ldots$. . . . . . . . 46

3.12 Order star do método $(4,2)$ de Brown . . . . . . . . . . . . . 46

3.13 Order star do método $(5,2)$ de Brown $\ldots \ldots \ldots \ldots$. . . . . . . . 46

3.14 Order star do método $(10,2)$ de Brown . . . . . . . . . . . . . . . 46 
3.15 Order star do método $(11,2)$ de Brown $\ldots \ldots \ldots \ldots$. . . . . . . . 46

3.16 Order star do método $(12,2)$ de Brown . . . . . . . . . . . . . . 46

3.17 Order star do método $(6,3)$ de Brown . . . . . . . . . . . . . . . 49

3.18 Order star do método $(4,5)$ de Brown . . . . . . . . . . . . . . 49

3.19 Zeros de $\rho(z)$ para $K=3$ e $\rho^{\prime}(z)$ para $K=4, L=1 \ldots \ldots$. . . . . . 50

3.20 Zeros de $\rho(z)$ para $K=3$ e $\rho^{\prime}(z)$ para $K=4, L=2 \ldots \ldots \ldots$

3.21 Zeros de $\rho(z)$ para $K=3$ e $\rho^{\prime}(z)$ para $K=4, L=3 \ldots \ldots$. . . . . . 50

3.22 Order star do método $(7,1)$ de Brown $\ldots \ldots \ldots \ldots$. . . . . . . . . 52

3.23 Order star do método $(7,2)$ de Brown $\ldots \ldots \ldots \ldots$. . . . . . . . 52

3.24 Order star do método $(7,3)$ de Brown $\ldots \ldots \ldots \ldots$. . . . . . . . 52

3.25 Curvas soluções para $w_{+}$e $w_{-}$para o método BDF de 2 passos $\ldots \ldots \ldots$. . . . 52

3.26 Região de estabilidade do método BDF de 2 passos . . . . . . . . . . . . 53

3.27 Região de estabilidade do método BDF de 3 passos . . . . . . . . . . . . . 53

3.28 Região de estabilidade do método BDF de 4 passos . . . . . . . . . . . . . . . 53

3.29 Regiões de estabilidade para os métodos de Brown para $L=1$ e $K=2, \ldots, 6$. . 54

3.30 Curvas soluções do método $(2,2)$ de Brown $\ldots \ldots$. . . . . . . . . 55

3.31 Região de estabilidade do método $(2,2)$ de Brown $\ldots \ldots$. . . . . . . . . . 55

3.32 Região de estabilidade do método $(3,2)$ de Brown $\ldots \ldots \ldots$. . . . . . . . 55

3.33 Região de estabilidade do método $(4,2)$ de Brown . . . . . . . . . . . . . 55

3.34 Regiões de estabilidade para os métodos de Brown para $L=2$ e $K=3, \ldots, 10$. . 56

3.35 Curvas soluções do método $(2,3)$ de Brown $\ldots \ldots \ldots$. . . . . . . . . 56

3.36 Região de estabilidade do método $(2,3)$ de Brown . . . . . . . . . . . . 57

3.37 Região de estabilidade do método $(3,3)$ de Brown . . . . . . . . . . . . 57

3.38 Região de estabilidade do método $(4,3)$ de Brown $\ldots \ldots$. . . . . . . . . . . 57

3.39 Região de estabilidade do método $(2,4)$ de Brown . . . . . . . . . . . . . . . . . 57

3.40 Região de estabilidade do método $(3,4)$ de Brown . . . . . . . . . . . . . . 57

3.41 Região de estabilidade do método $(4,4)$ de Brown . . . . . . . . . . . . . . 57 


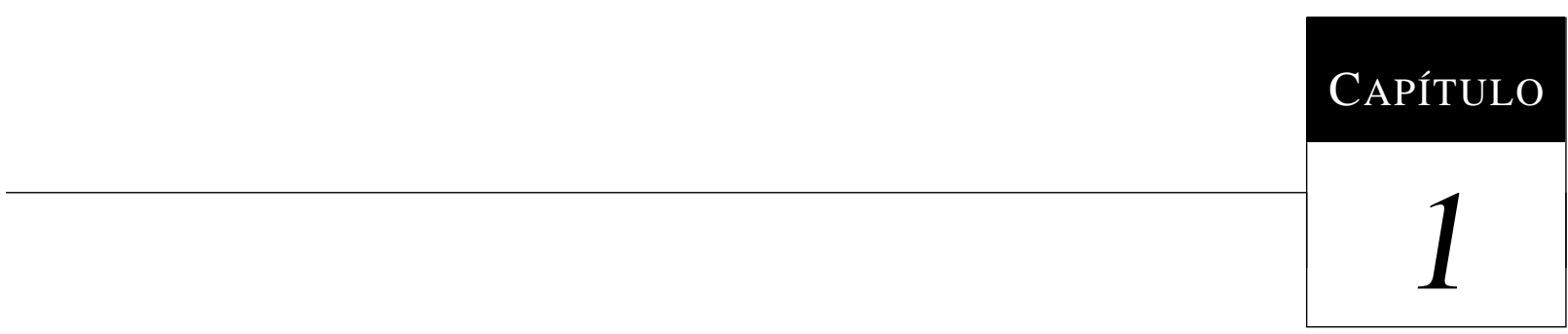

\section{Introdução}

A teoria das equações diferenciais tem sido muito utilizada nas últimas décadas, pois é uma área rica em aplicações não somente na Matemática mas também em outras ciências, como na Física e na Engenharia, por exemplo.

Neste trabalho, o enfoque principal será em questões relacionadas aos métodos numéricos para a solução de equações diferenciais ordinárias, com destaque para os métodos multiderivadas de passo múltiplo, que foram originalmente introduzidos com o intuito de obter métodos com menor número de passos e melhor precisão. A análise desses métodos desenvolveu-se paralelamente aos avanços em problemas stiff, que surgem quando são modelados fenômenos que envolvem repentinas mudanças de dinâmica ou variações bruscas como, por exemplo, em situações que envolvem reações nucleares e circuitos elétricos, entre outras. Esses fenômenos são, em geral, modelados por sistemas de equações diferenciais ordinárias lineares na forma

$$
\mathbf{y}^{\prime}=A \mathbf{y}+\phi(x)
$$

onde $A$ é uma matriz de ordem $m$ cujos autovalores $\lambda_{i}, i=1, \ldots, m$, são distintos e $\phi(x)$ é um vetor $m$-dimensional.

Os problemas stiff surgem quando a distância entre o maior e o menor autovalor da matriz $A$ é muito acentuada, sendo necessário o uso de dois valores muito diferentes de $h$ (tamanho do passo) para manter $z=\lambda h$ (obtida a partir da equação teste $y^{\prime}=\lambda y$ ) dentro da região de estabilidade do método numérico. 
A solução geral da equação (1.0.1) é

$$
\mathbf{y}(x)=\sum_{i=1}^{m} k_{i} e^{\lambda_{i} x} \mathbf{v}_{i}+\psi(x)
$$

onde $\mathbf{v}_{\mathbf{i}}$ é o autovetor correspondente ao autovalor $\lambda_{i}, i=1, \ldots, m$.

Assumindo que $\operatorname{Re}\left(\lambda_{i}\right)<0, i=1, \ldots, m$, segue que

$$
\lim _{x \rightarrow \infty} \sum_{i=1}^{m} k_{i} e^{\lambda_{i} x} \mathbf{v}_{i}=0
$$

O termo $\sum_{i=1}^{m} k_{i} e^{\lambda_{i} x} \mathbf{v}_{i}$ é chamado de solução transiente e $\psi(x)$ recebe o nome de solução de estado estacionário.

O exemplo a seguir ilustra as dificuldades presentes quando tenta-se obter uma solução aproximada do problema por métodos numéricos.

Exemplo 1.1. Sejam $\mathbf{y}=\left(\begin{array}{l}u \\ v\end{array}\right), A=\left(\begin{array}{cc}-2000 & 999,75 \\ 1 & -1\end{array}\right)$ e $\phi=\left(\begin{array}{c}1000,25 \\ 0\end{array}\right)$.

A equação (1.0.1) toma a forma

$$
\left(\begin{array}{l}
u \\
v
\end{array}\right)^{\prime}=\left(\begin{array}{cc}
-2000 & 999,75 \\
1 & -1
\end{array}\right)\left(\begin{array}{l}
u \\
v
\end{array}\right)+\left(\begin{array}{c}
1000,25 \\
0
\end{array}\right) \text {. }
$$

A condição inicial a ser utilizada é

$$
\left(\begin{array}{l}
u(0) \\
v(0)
\end{array}\right)=\left(\begin{array}{c}
0 \\
-2
\end{array}\right)
$$

Os autovalores da matriz $A$ são $\lambda_{1}=-2000,5$ e $\lambda_{2}=-0,5$, e, portanto, a solução exata é dada por

$$
u(x)=-1,499875 e^{-0,5 x}+0,499875 e^{-2000,5 x}+1
$$

$\mathrm{e}$

$$
v(x)=-2,99975 e^{-0,5 x}-0,00025 e^{-2000,5 x}+1 .
$$

A Figura 1.1 mostra o gráfico das soluções $u(x)$ e $v(x)$.

$\mathrm{Na}$ tentativa de obter uma solução aproximada do problema por métodos numéricos, uma dificuldade que surge é que, como $\lambda_{1}$ e $\lambda_{2}$ têm magnitudes muito diferentes, a necessidade de manter $z=\lambda h$ dentro da região de estabilidade leva a dois valores muito distintos de $h$, pois $z=\lambda_{1} h$ exige $h$ muito pequeno e $z=\lambda_{2} h$ permite uma passo maior. Portanto, o fato de alguns autovalores da matriz $A$ serem de grande magnitude e ficarem fora da região de estabilidade faz com que o tamanho do passo seja reduzido para garantir a estabilidade. 


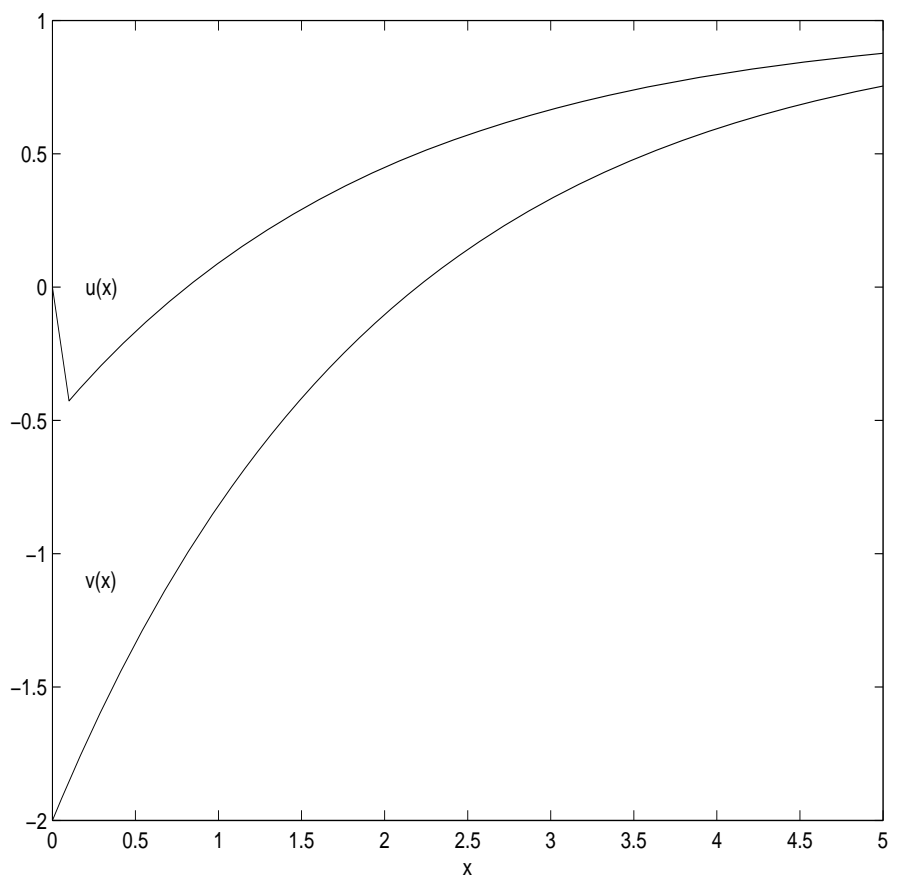

Figura 1.1: Soluções $u(x)$ e $v(x)$.

Os problemas onde os autovalores têm $\operatorname{Re}(\lambda)$ grande mas positiva também terão $z$ fora da região de estabilidade absoluta do método, mas estes casos não são interessantes, pois a solução exata cresce exponencialmente de qualquer forma (assim como o erro), não justificando o mesmo cuidado das situações em que $\operatorname{Re}(\lambda)<0$.

De maneira geral, ao analisar a solução transiente e a solução de estado estacionário, as seguintes características podem ser observadas:

1. se $\operatorname{Re}\left(\lambda_{i}\right)<0, i=1, \ldots, m$, está garantida a existência da solução de estado estacionário $\psi(x)$

2. se $\left|\operatorname{Re}\left(\lambda_{i}\right)\right|$ assume um valor pequeno para algum $i=1, \ldots, m$, como é o caso de $\lambda_{2}$ do Exemplo 1.1, obtém-se um vasto intervalo de integração para alcançar o estado estacionário, pois quanto menor $\left|\operatorname{Re}\left(\lambda_{i}\right)\right|$ o decaimento exponencial dado por $e^{\lambda_{i} x}$ será mais lento;

3. se $\left|\operatorname{Re}\left(\lambda_{i}\right)\right|$ assume um valor grande para algum $i=1, \ldots, m$, por exemplo $\lambda_{1}=-2000,5$, será necessário usar tamanho de passo pequeno para manter a estabilidade do método numérico;

4. quanto maior o quociente $\frac{\max _{i=1,2, \ldots, m}\left|\operatorname{Re}\left(\lambda_{i}\right)\right|}{\min _{i=1,2, \ldots, m}\left|\operatorname{Re}\left(\lambda_{i}\right)\right|}$, maior o esforço computacional gasto; este quociente recebe o nome de razão de stiffness.

Logo, a seguinte definição pode ser apresentada:

Definição 1.1. O sistema linear (1.0.1) é chamado de stiff se, para $i=1, \ldots, m, \operatorname{Re}\left(\lambda_{i}\right)<0 e$ $\max _{i=1,2, \ldots, m}\left|\operatorname{Re}\left(\lambda_{i}\right)\right|>>\min _{i=1,2, \ldots, m}\left|\operatorname{Re}\left(\lambda_{i}\right)\right|$, onde $\lambda_{i}, i=1,2, \ldots, m$, são os autovalores de $A$. 
Portanto, os métodos numéricos mais adequados para a solução de problemas stiff são aqueles que possuem grande região de estabilidade absoluta, pois assim existe maior liberdade na escolha do tamanho do passo.

Por possuírem boas propriedades de estabilidade, como grandes regiões de estabilidade absoluta, os métodos multiderivadas de passo múltiplo são indicados para resolver problemas stiff.

Existem muitos problemas que requerem a utilização de métodos numéricos com boas propriedades de estabilidade. Portanto, o estudo da estabilidade de métodos numéricos é uma área que possui grande potencial em pesquisa.

Uma classe de métodos multiderivadas de alta ordem, chamada de métodos $(K, L)$ de Brown, foi apresentada em Brown (1977) e caracteriza-se por possuir uma grande subclasse de métodos $A$-estáveis. A construção explícita e a dedução de algumas propriedades importantes desta classe foram apresentadas por Jeltsch e Kratz (1978).

Os métodos $(K, L)$ de Brown são definidos por

$$
\sum_{i=0}^{K} \alpha_{i} y_{n+i}=\sum_{j=1}^{L} \beta_{j} h^{j} f_{n+K}^{(j-1)}
$$

onde as constantes $\alpha_{i}$ e $\beta_{j}$ são escolhidas de modo a maximizar a precisão do método.

Os coeficientes $\alpha_{i}$ e $\beta_{j}$ são dados por

$$
\begin{aligned}
\alpha_{i} & =(-1)^{K-i}\left(\begin{array}{c}
K \\
i
\end{array}\right)(K-i)^{-L}, i=0, \ldots, K-1, \\
\alpha_{K} & =-\left(\alpha_{0}+\alpha_{1}+\ldots+\alpha_{K-1}\right), \\
\beta_{j} & =\frac{(-1)^{j}}{j !} \sum_{i=0}^{K-1}(-1)^{K-i}\left(\begin{array}{c}
K \\
i
\end{array}\right)(K-i)^{j-L}, j=1, \ldots, L .
\end{aligned}
$$

A constante do erro é representada por

$$
C_{K+L}=\frac{(-1)^{L} K !}{(K+L) !}
$$

Tais coeficientes foram obtidos em Jeltsch e Kratz (1978) através de representações matriciais, descritas a seguir:

$$
\left(\begin{array}{cc}
D 1_{L+1 \times K} & I_{L+1 \times L+1} \\
D 2_{K \times K} & 0_{K \times L+1}
\end{array}\right)\left(\begin{array}{c}
\underline{\alpha} \\
\underline{\beta}
\end{array}\right)=\Theta_{L+K+1 \times 1},
$$

onde $\underline{\alpha}=\left(\alpha_{0}, \alpha_{1}, \ldots, \alpha_{K-1}\right)^{T}, \underline{\beta}=\left(\beta_{0}, \beta_{1}, \ldots, \beta_{L}\right)^{T}$, $\Theta$ é a matriz nula, $I_{r \times r}$ é a matriz identidade de ordem $r$ e $D_{L+K+1 \times K}=\left(\begin{array}{cc}D 1 & D 2\end{array}\right)^{T}$ é definida por $d_{i, j}=\frac{(j-K)^{i}}{i !}$, para $0 \leq i \leq L+K$ e $0 \leq j \leq K-1$. 
Quando $L=1$, a classe de métodos de Brown e os métodos BDF (Backward Difference Formulae) representam a mesma classe de métodos, sendo este aspecto não verificado por Jeltsch em seus estudos. A seguir este fato é mostrado através das fórmulas de interpolação de Newton (nós simples), no caso em que $L=1$, e de Hermite (nós múltiplos), para $L \geq 2$. Maiores detalhes a respeito dessas fórmulas de interpolação podem ser encontrados em Hairer e Wanner (1996).

Uma representação para os métodos $(K, L)$ de Brown para $L=1$ pode ser encontrada por meio da fórmula de interpolação de Newton nos pontos $x_{n+1}, x_{n}, \ldots, x_{n+1-K}$, derivando o polinômio resultante no ponto $x_{n+1}$. De fato, das diferenças divididas, segue que

$$
\begin{array}{ccccccc}
s=1 & f_{1} & & & & \\
s=0 & f_{0} & & \frac{\nabla^{2} f_{1}}{2 !} & & \\
& & \nabla f_{0} & & \frac{\nabla^{3} f_{1}}{3 !} & \\
s=-1 & f_{-1} & & \frac{\nabla^{2} f_{0}}{2 !} & & \frac{\nabla^{4} f_{1}}{4 !} \\
& & \nabla f_{-1} & & \frac{\nabla^{3} f_{0}}{3 !} & \vdots \\
s=-2 & f_{-2} & & \frac{\nabla^{2} f_{-1}}{2 !} & \vdots & \\
& & \nabla f_{-2} & \vdots & & \\
s=-3 & f_{-3} & \vdots & & &
\end{array}
$$

onde $x=x_{n}+s h, f_{1}=f\left(x_{n}+h\right)$ e $\nabla^{j}$ denota a $j$-ésima diferença para trás.

Então

$$
\begin{aligned}
f\left(x_{n}+s h\right)= & f_{1}+(s-1) \nabla f_{1}+(s-1) s \frac{\nabla^{2} f_{1}}{2 !}+(s-1) s(s+1) \frac{\nabla^{3} f_{1}}{3 !} \\
& +(s-1) s(s+1)(s+2) \frac{\nabla^{4} f_{1}}{4 !}+\ldots
\end{aligned}
$$

Substituindo $f$ por $y$,

$$
\begin{aligned}
y\left(x_{n}+s h\right)= & y_{1}+(s-1) \nabla y_{1}+(s-1) s \frac{\nabla^{2} y_{1}}{2 !}+(s-1) s(s+1) \frac{\nabla^{3} y_{1}}{3 !} \\
& +(s-1) s(s+1)(s+2) \frac{\nabla^{4} y_{1}}{4 !}+\ldots
\end{aligned}
$$

Derivando no ponto $s=1$, segue que

$$
h f_{n+1}=\sum_{j=1}^{K} \frac{1}{j} \nabla^{j} y_{n+1},
$$

que é a fórmula dos métodos BDF. 
Para encontrar os coeficientes para alguns valores fixos de $K$ dos métodos $(K, 1)$, basta utilizar a expressão (1.0.2). Por exemplo, para $K=2$, segue, de (1.0.2),

$$
\begin{aligned}
h f_{n+1} & =\sum_{j=1}^{2} \frac{1}{j} \nabla^{j} y_{n+1}=\nabla y_{n+1}+\frac{1}{2} \nabla^{2} y_{n+1}=\nabla y_{n+1}+\frac{1}{2}\left(\nabla y_{n+1}-\nabla y_{n}\right) \\
& =\frac{3}{2} \nabla y_{n+1}-\frac{1}{2} \nabla y_{n}=\frac{3}{2}\left(y_{n+1}-y_{n}\right)-\frac{1}{2}\left(y_{n}-y_{n-1}\right)=\frac{3}{2} y_{n+1}-2 y_{n}+\frac{1}{2} y_{n-1} .
\end{aligned}
$$

Portanto, o método $(2,1)$ de Brown é definido por

$$
\frac{3}{2} y_{n+1}-2 y_{n}+\frac{1}{2} y_{n-1}=h f_{n+1}
$$

Para $L=2$ a mesma idéia do caso $L=1$ pode ser aplicada, mas considerando a fórmula de interpolação de Hermite nos pontos $x_{n+1}$ (nó duplo), $x_{n}, x_{n-1}, \ldots, x_{n+1-K}$ (nós simples) e derivando o polinômio resultante duas vezes em $x=x_{n+1}$. Então, das diferenças divididas,

$$
\begin{array}{cccccc}
s=1 & f_{1} & & & & \\
s=1 & f_{1} & & h f_{1}^{\prime}-\nabla f_{1} & & \\
& & \nabla f_{1} & & \frac{h f_{1}^{\prime}-\nabla f_{1}-\frac{1}{2} \nabla^{2} f_{1}}{2 !} & \\
s=0 & f_{0} & & \frac{\nabla^{2} f_{1}}{2 !} & & \frac{h f_{1}^{\prime}-\nabla f_{1}-\frac{1}{2} \nabla^{2} f_{1}-\frac{1}{3} \nabla^{3} f_{1}}{3 !} \\
& & \nabla f_{0} & & \frac{\nabla^{3} f_{1}}{3 !} & \vdots \\
s=-1 & f_{-1} & & \frac{\nabla^{2} f_{0}}{2 !} & \vdots & \\
& & \nabla f_{-1} & \vdots & & \\
s=-2 & f_{-2} & \vdots & &
\end{array}
$$

Logo

$$
\begin{aligned}
f\left(x_{n}+s h\right)= & f_{1}+(s-1) h f_{1}^{\prime}+(s-1)^{2}\left(h f_{1}^{\prime}-\nabla f_{1}\right)+(s-1)^{2} s \frac{h f_{1}^{\prime}-\nabla f_{1}-\frac{1}{2} \nabla^{2} f_{1}}{2 !} \\
& +(s-1)^{2} s(s+1) \frac{h f_{1}^{\prime}-\nabla f_{1}-\frac{1}{2} \nabla^{2} f_{1}-\frac{1}{3} \nabla^{3} f_{1}}{3 !}+\ldots
\end{aligned}
$$

Substituindo $f$ por $y$,

$$
\begin{aligned}
y\left(x_{n}+s h\right)= & y_{1}+(s-1) h y_{1}^{\prime}+(s-1)^{2}\left(h y_{1}^{\prime}-\nabla y_{1}\right)+(s-1)^{2} s \frac{h y_{1}^{\prime}-\nabla y_{1}-\frac{1}{2} \nabla^{2} y_{1}}{2 !} \\
& +(s-1)^{2} s(s+1) \frac{h y_{1}^{\prime}-\nabla y_{1}-\frac{1}{2} \nabla^{2} y_{1}-\frac{1}{3} \nabla^{3} y_{1}}{3 !}+\ldots
\end{aligned}
$$


Derivando duas vezes no ponto $s=1$ e considerando $y^{\prime \prime}\left(x_{n+1}\right)=g_{n+1}$, segue que

$$
\frac{h^{2}}{2} g_{n+1}=\left(\sum_{i=1}^{K} \frac{1}{i}\right) h f_{n+1}-\sum_{j=1}^{K}\left(\sum_{i=j}^{K} \frac{1}{i}\right) \frac{\nabla^{j} y_{n+1}}{j} .
$$

Tal expressão foi usada por Hairer e Wanner (1996) para representar os "second derivative BDF methods (SDBDF)". Para encontrar alguns coeficientes dos métodos $(K, 2)$ de Brown para valores fixos de $K$ basta utilizar a expressão (1.0.3).

O mesmo procedimento pode ser usado para determinar as expressões para outros métodos $(K, L)$ para valores fixos de $L$, bastando utilizar a interpolação de Hermite nos pontos $x_{n+1}$ (nó de multiplicidade $L$ ), $x_{n}, x_{n-1}, \ldots, x_{n+1-K}$ (nós simples) e derivar o polinômio resultante $L$ vezes em $x=x_{n+1}$. Além disso, alguns coeficientes para valores fixos de $K$ e $L$ dos métodos $(K, L)$ encontram-se em Meneguette (1987). Portanto, de maneira geral, os métodos $(K, L)$ de Brown podem ser chamados de "multiderivative BDF methods (MDBDF)".

Jeltsch, em uma série de artigos, estudou os métodos $(K, L)$ e provou alguns resultados assintóticos como, por exemplo, o comportamento desses métodos quando $K$ cresce e $L$ fica fixo, quando $K$ está fixo e $L$ cresce e apresentou também o resultado:

Teorema 1.1. Os métodos $(K, L)$ de Brown são stiff-estáveis se, e somente se, são $A_{0}$ e fortemente estáveis.

Um resultado interessante demonstra que $A_{0}$-estabilidade dos métodos $(K, L)$ está relacionada ao polinômio

$$
\rho_{\gamma}(z)=\left(\alpha_{K}+\gamma\right) z^{K}+\alpha_{K-1} z^{k-1}+\ldots+\alpha_{0},
$$

com $\gamma$ um parâmetro real variando de 0 a $\infty$ e $\rho_{0}(z)$ sendo o polinômio característico do método $(K, L)$ que fornece a zero-estabilidade. Esse resultado parece uma forte indicação de que é possível refazer o teorema anterior sem a exigência da $A_{0}$-estabilidade. Conjectura-se, então,

Conjectura 1.1. Todos os métodos $(K, L)$ de Brown são stiff-estáveis se, e somente se, são zeroestáveis.

Ou, equivalentemente, "um método $(K, L)$ zero-estável é stiff-estável".

No caso dos métodos BDF tal resultado é válido, pois Hairer e Wanner (1983) mostraram que tais métodos são zero-estáveis para $K \leq 6$ e também $A_{0}$-estáveis.

Em Meneguette (1987), demonstrou-se que os coeficientes de $\rho_{\gamma}(z)$ são positivos, satisfazendo,

1. $0<\alpha_{0}<\alpha_{1}<\ldots<\alpha_{K-1}$ mas $\alpha_{K}<\alpha_{K-1}$, para $K \leq K_{L}$;

2. $2 \alpha_{j}<\alpha_{j+1}$ para $0 \leq j \leq K_{L}^{*}$; 
onde

$$
K_{L}=\min \left\{2^{L+1}+1, \frac{3^{L+1}}{2^{L}}+2, \ldots, \frac{(L+1)^{L+1}}{L^{L}}+L\right\}
$$

$\mathrm{e}$

$$
K_{L}^{*}=\min \left\{2^{L}+1, \frac{3^{L+1}}{2^{L+1}}+2, \ldots, \frac{(L+1)^{L+1}}{2 L^{L}}+L\right\} .
$$

Se $\gamma>\alpha_{K-1}-\alpha_{K}$, todos os zeros de $\rho_{\gamma}(z)$ encontram-se no disco unitário, como conseqüência do Teorema de Eneström-Kakeya. Se $0<\gamma<\alpha_{K-1}-\alpha_{K}$, o comportamento dos zeros ainda não é conhecido. De uma maneira geral, a seguinte conjectura pode ser estabelecida:

Conjectura 1.2. Seja $P(z)=a_{n} z^{n}+a_{n-1} z^{n-1}+\ldots+a_{0}$ um polinômio tal que

$$
0<a_{0} \leq a_{1} \leq \ldots \leq a_{n-1} \text { e } a_{n}<a_{n-1},
$$

cujos zeros encontram-se no disco unitário, e $P^{\prime}(z)$ com os coeficientes ordenados. Então, os zeros do polinômio

$$
P_{\gamma}(z)=\left(a_{n}+\gamma\right) z^{n}+a_{n-1} z^{n-1}+\ldots+a_{0}
$$

encontram-se no disco unitário, para todo $\gamma>0$.

Esse é um problema que encontra-se em aberto em Meneguette (1994). A origem dessa conjectura está relacionada aos polinômios característicos associados aos métodos $(K, L)$ de Brown, mas sua formulação é válida para um polinômio qualquer que satisfaz as condições descritas acima.

Através dessa conjectura, muitos problemas sobre estabilidade dos métodos $(K, L)$ de Brown podem ser resolvidos, como o resultado que afirma que, para $K \leq K_{L}$, os métodos $(K, L)$ de Brown são stiff-estáveis. Portanto, um dos principais objetivos deste trabalho é o estudo da estabilidade dos métodos $(K, L)$ de Brown, pois muitas questões relacionadas à estabilidade desta classe de métodos ainda não foram exploradas. O foco principal é a análise das conjecturas, sendo que, para estudá-las, são utilizados resultados clássicos da literatura, como o Teorema de EneströmKakeya, por exemplo. Além disso, a construção das regiões de estabilidade para alguns valores fixos de $K$ e $L$ dos métodos $(K, L)$ é um item importante para a solução de alguns problemas. Convém lembrar que o estudo da Conjectura 1.2 é importante nas questões de estabilidade dos métodos $(K, L)$, mas também é significativo na área de zeros de polinômios.

Outra ferramenta que tem extrema importância nas questões relacionadas à estabilidade são as order stars, que foram introduzidas por Wanner et al. (1978), através das quais é possível descrever ordem, estabilidade e suas relações como propriedades de certas funções complexas. A teoria das order stars ainda é pouco conhecida e divulgada, pois seu desenvolvimento é recente quando comparado a outras áreas.

Para o desenvolvimento do trabalho é apresentada, no Capítulo 2, uma análise da Conjectura 1.2. Tal análise baseia-se em resultados clássicos da área de zeros de polinômios, que são enunciados no início do Capítulo 2. Depois é encontrado um limitante para os zeros de $P_{\gamma}(z)$, ou seja, os 
zeros de $P_{\gamma}(z)$ encontram-se em

$$
|z| \leq 1+\frac{1}{n-1}
$$

Em seguida é mostrada a validade da Conjectura 1.2 no caso em que o polinômio $P(z)$ é reflexivo, estabelecendo uma condição sobre $\gamma$ para que o polinômio $P_{\gamma}(z)$, sujeito apenas às condições de $P(z)$ ter seus zeros no disco, cujos coeficientes satisfazem as desigualdades descritas na conjectura, tenha também seus zeros no disco unitário. Esta consideração representa um caso crítico da conjectura, pois $P(z)$ possui todos os seus zeros sobre o círculo unitário e qualquer perturbação dos coeficientes poderia fazer os zeros saírem do disco unitário. Mas, como é possível observar no Capítulo 2, a perturbação no coeficiente dominante faz com que os zeros de $P_{\gamma}(z)$ permaneçam no disco unitário. Além disso, é analisada a Conjectura 1.2 no caso em que os coeficientes de $P(z)$ satisfazem algumas condições adicionais, como, por exemplo, quando são verdadeiras as seguintes desigualdades:

$$
p a_{i}<(p-1) a_{i+1}, i=0,1, \ldots, n-2, \text { e } p a_{n}>(p-1) a_{n-1}
$$

onde $p$ é um número inteiro positivo, $p>1$. Nesse caso, a Conjectura 1.2 é válida. Portanto, no Capítulo 2, é realizado um estudo da Conjectura 1.2 no caso geral, não necessariamente no caso em que os polinômios analisados são os polinômios característicos associados aos métodos $(K, L)$ de Brown, sendo esse caso objeto de estudo do Capítulo 3.

Para $K \leq K_{L}^{*}$, os coeficientes de $\rho(z)$ satisfazem

$$
2 \alpha_{j}<\alpha_{j+1} \text { e } 2 \alpha_{K}>\alpha_{K-1}
$$

Portanto, no Capítulo 3, mostra-se que a Conjectura 1.2 é verdadeira para $K \leq K_{L}^{*}$, sendo $P(z)=\rho(z)$, ou seja, o polinômio característico associado ao método $(K, L)$ de Brown, onde $K$ e $L$ são fixos. Através desse resultado segue que a Conjectura 1.1 é válida para $K \leq K_{L}^{*}$, que é um resultado de significativa importância na área.

Além disso, no Capítulo 3, é apresentado um estudo sobre order stars, trazendo uma generalização desse conceito para o caso dos métodos $(K, L)$ de Brown, que são métodos multiderivadas de passo múltiplo, sendo o caso dos métodos lineares de passo múltiplo analisado em Iserles e Norsett (1984).

A teoria das order stars é uma ferramenta que será muito utilizada na análise das questões de estabilidade dos métodos $(K, L)$. Por exemplo, através das order stars, prova-se que os zeros dos polinômios característicos associados aos métodos $(K, L)$ são distintos. Outras propriedades dos métodos $(K, L)$ que são abordadas através das order stars são as seguintes: para $L$ fixo e $K$ suficientemente grande, os métodos $(K, L)$ tornam-se instáveis; para $K$ fixo e $L$ suficientemente grande, tornam-se zero-estáveis. As regiões de estabilidade dos métodos $(K, L)$ para alguns valores de $K$ e $L$ fixos, que ainda não foram publicadas na literatura, são construídas, assim como as order stars de tais métodos. 
Portanto, é dado um enfoque diferente às propriedades já conhecidas dos métodos $(K, L)$ de Brown, demonstrando-as através das order stars, e também através desta ferramenta mostra-se que os zeros dos polinômios característicos associados aos métodos $(K, L)$ são distintos, que é um comportamento dos zeros que não foi provado na literatura sendo, então, um resultado importante.

Para finalizar, um capítulo com as conclusões é apresentado, enfatizando os principais resultados obtidos neste trabalho e apresentando alguns assuntos que ainda podem ser pesquisados e explorados. 
Este capítulo traz uma revisão bibliográfica sobre localização de zeros de polinômios e também um estudo da conjectura apresentada no capítulo anterior. Primeiramente são apresentados resultados clássicos sobre a localização de zeros de polinômios, assunto que será muito utilizado na análise das conjecturas objetos de estudo desta tese. Em seguida, uma seção traz uma análise da Conjectura 1.2, onde, primeiramente, é determinado um limitante para os zeros de $P_{\gamma}(z)$. Depois é mostrada a validade da conjectura no caso em que o polinômio é reflexivo. Em seguida, uma análise da conjectura no caso em que os coeficientes de $P_{\gamma}(z)$ satisfazem algumas condições adicionais é apresentada.

\subsection{Resultados clássicos sobre zeros de polinômios}

Nesta seção são apresentados resultados importantes que delimitam regiões onde estão localizados os zeros de polinômios. Esses resultados foram muito importantes para o desenvolvimento da pesquisa, pois para determinar a zero-estabilidade de um método numérico é preciso analisar a quantidade de zeros no disco unitário.

\subsubsection{Localização dos zeros}

Os teoremas apresentados a seguir são resultados clássicos da literatura e indicam regiões onde estão localizados os zeros de um polinômio. Um estudo detalhado do assunto encontra-se em Botta (2003), Marden (1966) e Milovanóvic et al. (1994). 
A seguir é apresentado um resultado clássico sobre a localização dos zeros de um polinômio, que é o Teorema de Eneström-Kakeya, que encontra-se em Marden (1966).

Teorema 2.1 (Eneström-Kakeya). Seja $P(z)=\sum_{i=0}^{n} a_{i} z^{i}$ um polinômio cujos coeficientes $a_{i}$, $i=0,1, \ldots, n$, satisfazem

$$
0<a_{0} \leq a_{1} \leq \ldots \leq a_{n}
$$

Então, $P(z)$ possui seus zeros em $|z| \leq 1$.

No próximo teorema é determinado um anel que contém todos os zeros de um polinômio. Este resultado, que é um caso mais abrangente do Teorema de Eneström-Kakeya, encontra-se em Anderson et al. (1979).

Teorema 2.2. Seja $P(z)=\sum_{i=0}^{n} a_{i} z^{i}$ um polinômio tal que $n \geq 1$ e $a_{k}>0$ para $k=0,1, \ldots, n$. Considerando

$$
\alpha=\min _{0 \leq k<n}\left\{\frac{a_{k}}{a_{k+1}}\right\} e \beta=\max _{0 \leq k<n}\left\{\frac{a_{k}}{a_{k+1}}\right\}
$$

todos os zeros de $P(z)$ encontram-se no anel $\alpha \leq|z| \leq \beta$.

São utilizadas também as fórmulas de Vieta, que determinam relações entre os zeros de um polinômio e seus coeficientes, dadas por:

$$
\begin{aligned}
z_{1} z_{2} \ldots z_{n} & =(-1)^{n} \frac{a_{0}}{a_{n}} \\
z_{1} z_{2} \ldots z_{n-1}+\ldots+z_{2} z_{3} \ldots z_{n} & =(-1)^{n-1} \frac{a_{1}}{a_{n}} \\
& \vdots \\
z_{1}+z_{2}+\ldots+z_{n} & =-\frac{a_{n-1}}{a_{n}},
\end{aligned}
$$

onde $z_{1}, z_{2}, \ldots, z_{n}$ são os zeros do polinômio $P(z)=\sum_{i=0}^{n} a_{i} z^{i}$.

Existem muitos resultados que relacionam os zeros de um polinômio com os de sua derivada. $\mathrm{O}$ resultado a seguir, que encontra-se em Marden (1966), recebe o nome de Teorema de Gauss-Lucas e representa uma generalização do Teorema de Rolle.

Teorema 2.3 (Gauss-Lucas). Os zeros da derivada de um polinômio estão contidos na região convexa fechada formada pelos zeros desse polinômio.

Como consequiência do Teorema de Gauss-Lucas tem-se o seguinte resultado.

Teorema 2.4. Qualquer disco que contém todos os zeros de um polinômio também contém todos os zeros da derivada desse polinômio. 
A seguir é analisado um algoritmo, chamado de algoritmo de Schur, e alguns resultados sobre a quantidade de zeros no disco unitário, sendo tal abordagem apresentada em Marden (1948).

Suponha agora que o polinômio $P(z)=\sum_{i=0}^{n} a_{i} z^{i}, a_{i} \in \mathbb{R}$, tenha $p(p \leq n)$ zeros em $|z| \leq 1$.

Associado a ele considere o polinômio

$$
P^{*}(z)=z^{n} P\left(\frac{1}{z}\right)=a_{0} z^{n}+a_{1} z^{n-1}+\ldots+a_{n}=a_{0} \prod_{j=1}^{n}\left(z-z_{j}^{*}\right)
$$

sendo seus zeros $z_{k}^{*}$, em relação ao disco unitário, os inversos dos zeros $z_{k}$ de $P(z)$, isto é, $z_{k}^{*}=\frac{1}{z_{k}}$.

Logo, qualquer zero de $P(z)$ que possui módulo um é também zero de $P^{*}(z)$ e se $P(z)$ não tem zeros em $|z|=1, P^{*}(z)$ também não tem zeros em $|z|=1$. Além disso, se $P(z)$ possui $p$ zeros em $|z|<1$, então $P^{*}(z)$ possui $n-p$ zeros em $|z|<1$.

Seja agora a seqüência de polinômios

$$
P_{j}(z)=\sum_{k=0}^{n-j} a_{k}^{(j)} z^{k}
$$

onde $P_{0}(z)=P(z)$ e

$$
P_{j+1}(z)=a_{0}^{(j)} P_{j}(z)-a_{n-j}^{(j)} P_{j}^{*}(z)
$$

$\operatorname{com} j=0, \ldots, n-1$.

Então,

$$
a_{k}^{(j+1)}=a_{0}^{(j)} a_{k}^{(j)}-a_{n-j}^{(j)} a_{n-j-k}^{(j)} .
$$

Em cada polinômio $P_{j}(z)$ o termo constante $a_{0}^{(j)}$ é representado por $\delta_{j}$. Assim,

$$
\delta_{j+1}=\left|a_{0}^{(j)}\right|^{2}-\left|a_{n-j}^{(j)}\right|^{2}=a_{0}^{(j+1)},
$$

$\operatorname{com} j=0, \ldots, n-1$.

Os próximos resultados relacionam a quantidade de zeros de $P_{j}(z)$ e $P_{j}^{*}(z)$ em relação ao disco unitário e são muito utilizados na análise da Conjectura 1.2.

Lema 2.1. Se $P_{j}$ tem $p_{j}$ zeros em $|z| \leq 1$ e se $\delta_{j+1} \neq 0$, então $P_{j+1}$ tem

$$
p_{j+1}=\frac{1}{2}\left\{n-j-\left[(n-j)-2 p_{j}\right] \operatorname{sinal}\left(\delta_{j+1}\right)\right\}
$$

zeros em $|z| \leq 1$. Além disso, $P_{j+1}$ tem os mesmos zeros em $|z|=1$ que $P_{j}$.

A demonstração desse lema pode ser encontrada em Marden (1966). 
Teorema 2.5. Se $\left|a_{0}\right|<\left|a_{n}\right|, P(z)$ tem todos os seus zeros no disco unitário se, e somente se, $P_{1}^{*}$ tem também todos os seus zeros em $|z| \leq 1$.

A demonstração desse resultado segue do Lema 2.1.

Proposição 2.1. Se os coeficientes de $P(z)$ são todos reais e se $0<a_{n}<a_{n-1}<\ldots<a_{1}<a_{0}$, então todos os $a_{k}^{(j)}$ dados por (2.1.1) são reais e $0<a_{n-j}^{(j)}<a_{n-j-1}^{(j)}<\ldots<a_{0}^{(j)}$, para $j=1, . ., n$.

Através desse resultado é possível determinar a quantidade de zeros dos polinômios $P_{j}^{*}(z)$ no disco unitário, como decorrência do Teorema de Eneström-Kakeya.

A seguir tem-se o critério de Schur-Cohn, que determina a quantidade de zeros de um polinômio no interior do círculo unitário através de condições expressas em termos dos coeficientes de $P(z)$.

Teorema 2.6 (Critério de Schur-Cohn). Seja $P(z)=\sum_{i=0}^{n} a_{i} z^{i}$. Se os determinantes

$$
\Delta_{k}=\left|\begin{array}{ccccccc}
a_{0} & 0 & \ldots & 0 & a_{n} & \ldots & a_{n-k+1} \\
a_{1} & a_{0} & \ldots & 0 & 0 & \ldots & a_{n-k+2} \\
\vdots & \vdots & \ldots & \vdots & \vdots & \ldots & \vdots \\
a_{k-1} & a_{k-2} & \ldots & a_{0} & 0 & \ldots & a_{n} \\
\bar{a}_{n} & 0 & \ldots & 0 & \bar{a}_{0} & \ldots & \bar{a}_{k-1} \\
\bar{a}_{n-1} & \bar{a}_{n} & \ldots & 0 & 0 & \ldots & \bar{a}_{k-2} \\
\vdots & \vdots & \ldots & \vdots & \vdots & \ldots & \vdots \\
\bar{a}_{n-k+1} & \bar{a}_{n-k+2} & \ldots & \bar{a}_{n} & 0 & \ldots & \bar{a}_{0}
\end{array}\right|, k=1,2, \ldots, n
$$

são diferentes de zero, então $P(z)$ não possui zeros em $|z|=1$ e p zeros em $|z|<1$, onde p é o número de variação de sinal da seqüência $1, \Delta_{1}, \ldots, \Delta_{n}$ e $\bar{a}_{i}$ representa o complexo conjugado de $a_{i}, i=0,1, \ldots, n$.

Como tal condição envolve o cálculo de determinantes de matrizes de altas ordens, sua utilização será limitada.

A seguir é apresentado o critério de Hurwitz. Sejam $P(z)=\sum_{i=0}^{n} a_{i} z^{i}$ e os determinantes

$$
D_{k}=\left|\begin{array}{cccc}
a_{1} & a_{3} & \ldots & a_{2 k-1} \\
a_{0} & a_{2} & \ldots & a_{2 k-2} \\
0 & a_{1} & \ldots & a_{2 k-3} \\
0 & a_{0} & \ldots & a_{2 k-4} \\
\vdots & \vdots & \ldots & \vdots \\
0 & 0 & \ldots & a_{k}
\end{array}\right|
$$

$k=2, \ldots, n$ e $D_{1}=a_{1}$.

O teorema a seguir é conhecido como critério de Hurwitz. 
Teorema 2.7. Se todos os determinantes $D_{k}$ são positivos, o polinômio $P(z)$ tem somente zeros com parte real negativa.

Definição 2.1. Os polinômios reais cujos zeros estão localizados no semi-plano esquerdo são chamados de polinômios de Hurwitz.

Esse critério pode ser utilizado para mostrar que os zeros de um polinômio encontram-se no disco unitário fazendo a transformação $r=\frac{1+z}{1-z}$, que mapeia o interior do disco unitário no semi-plano $\operatorname{Re}(z)<0$.

\subsection{Análise da Conjectura 1.2}

Nesta seção é abordado um estudo da Conjectura 1.2. Na primeira subseção é encontrado um limitante para os zeros de $P_{\gamma}(z)$. Depois é analisado o caso em que $P(z)$ é um polinômio reflexivo, com a apresentação de alguns exemplos. Em seguida, considerando um polinômio sujeito a algumas condições sobre seus coeficientes, é mostrada a validade da Conjectura 1.2.

\subsubsection{Limitante para os zeros}

Do Teorema 2.2, segue que

$$
\beta=\max _{0 \leq k<n}\left\{\frac{a_{k}}{a_{k+1}}\right\}=\frac{a_{n-1}}{a_{n}+\gamma} .
$$

Mas como $P^{\prime}(z)$ possui os coeficientes ordenados, então $P_{\gamma}^{\prime}(z)$ também possui. Logo, $n\left(a_{n}+\gamma\right)>(n-1) a_{n-1}$.

Portanto, do Teorema 2.2, segue que os zeros de $P_{\gamma}(z)$ encontram-se em

$$
|z| \leq \beta=\frac{a_{n-1}}{a_{n}+\gamma}<\frac{n}{n-1}=1+\frac{1}{n-1}
$$

Então, os zeros do polinômio $P_{\gamma}(z)$ encontram-se em

$$
|z|<1+\frac{1}{n-1}
$$

\subsubsection{Polinômio reflexivo}

Agora é analisado o caso em que o polinômio $P(z)$ é um polinômio reflexivo, isto é,

$$
a_{i}=a_{n-i}, a_{i}>0(i=0,1,2, \ldots, n)
$$


onde

$$
a_{0} \leq a_{1} \leq \ldots \leq a_{n-1} \text { e } a_{n-1}>a_{n}
$$

Mas como $P(z)$ satisfaz as hipóteses da Conjectura 1.2, segue que

$$
a_{0}<a_{1}=a_{2}=\ldots=a_{n-1} \text { e } a_{n-1}>a_{n} .
$$

Convém lembrar que os coeficientes apresentados acima são quaisquer, somente satisfazendo as condições da Conjectura $1.2 \mathrm{com} P(z)$ um polinômio reflexivo.

Através desta pesquisa foi possível chegar a um resultado mais amplo, não sendo necessário estabelecer que $P^{\prime}(z)$ tenha seus zeros no disco. Além disso, se $P(z)$ está sujeito a estas condições, seus zeros encontram-se sobre o disco unitário, sendo, portanto, um caso crítico da conjectura, já que qualquer perturbação dos coeficientes acarretaria numa perturbação dos zeros. Portanto, a seguir é exibida a demonstração de que, para qualquer $\gamma>0$, o polinômio $P_{\gamma}(z)$, sujeito às condições da Conjectura 1.2, possui seus zeros no disco unitário, e é encontrada uma condição sobre $\gamma$ para que o polinômio $P_{\gamma}(z)$, sujeito apenas à condição de $P(z)$ (cujos coeficientes satisfazem a ordenação acima) possuir todos os seus zeros no disco, tenha também seus zeros no disco unitário.

Seja $P(z)=\sum_{i=0}^{n} a_{i} z^{i}$ um polinômio que possui todos os seus zeros no disco unitário tal que

$$
a_{i}=a_{n-i}, a_{i}>0(i=0,1,2, \ldots, n)
$$

onde

$$
a_{0}<a_{1}=\ldots=a_{n-1} \text { e } a_{n-1}>a_{n} .
$$

Sejam

$$
P_{1}^{*}(z)=\sum_{i=0}^{n-1} a_{i}^{(1)} z^{n-1-i}
$$

(relacionado à $P(z)) \mathrm{e}$

$$
P_{1, \gamma}^{*}(z)=\sum_{i=0}^{n-1} a_{i, \gamma}^{(1)} z^{n-1-i}
$$

(relacionado à $P_{\gamma}(z)$ ), onde os coeficientes $a_{i}^{(1)}$ e $a_{i, \gamma}^{(1)}, i=0, \ldots, n-1$, são dados pela expressão (2.1.1).

Se $\gamma>0$, segue que $\left|a_{0}\right|<\left|a_{n}+\gamma\right|$. Caso contrário, tal hipótese é considerada. Assim, o Teorema 2.5 pode ser utilizado para $P_{\gamma}(z)$.

Observe que

$$
\left|a_{n-1, \gamma}^{(1)}\right|=\left|a_{n-2, \gamma}^{(1)}\right|=\ldots=\left|a_{1, \gamma}^{(1)}\right| .
$$


Resta analisar a relação existente entre $\left|a_{0, \gamma}^{(1)}\right|$ e $\left|a_{1, \gamma}^{(1)}\right|$ :

$$
\left|a_{0, \gamma}^{(1)}\right|-\left|a_{1, \gamma}^{(1)}\right|=|\gamma|\left(\gamma+2 a_{n}-a_{n-1}\right)
$$

Se $\left|a_{0, \gamma}^{(1)}\right| \geq\left|a_{1, \gamma}^{(1)}\right|$, os coeficientes de $P_{1, \gamma}^{*}(z)$ estão ordenados e então, pelo Teorema de EneströmKakeya, os zeros de $P_{1, \gamma}^{*}(z)$ encontram-se no disco unitário. Mas para que isso ocorra é necessário que

$$
|\gamma|\left(\gamma+2 a_{n}-a_{n-1}\right) \geq 0 \Leftrightarrow \gamma \geq a_{n-1}-2 a_{n} .
$$

Portanto, para $\gamma \geq a_{n-1}-2 a_{n}$, o polinômio $P_{\gamma}(z)$ possui os zeros no disco unitário.

No caso do polinômio reflexivo estar sujeito às condições da Conjectura 1.2, isto é, sendo $P(z)$ um polinômio cujos zeros encontram-se no disco unitário e $P^{\prime}(z)$ com os coeficientes ordenados, segue que

$$
2 a_{n}-a_{n-1} \geq 0
$$

sendo $n>1$.

Logo, para qualquer $\gamma>0$, os coeficientes de $P_{1, \gamma}^{*}(z)$ estão ordenados.

Para analisar o caso em que $\gamma<a_{n-1}-2 a_{n}$, observe que

$$
\left|a_{0, \gamma}^{(1)}\right|-\left|a_{n-1, \gamma}^{(1)}\right|=\gamma\left(\gamma+2 a_{n}-a_{1}\right)=|\gamma|\left(\gamma+2 a_{n}-a_{n-1}\right)<0 .
$$

Portanto, $\left|a_{0, \gamma}^{(1)}\right|<\left|a_{n-1, \gamma}^{(1)}\right|$.

Utilizando uma das relações das fórmulas de Vieta, dada por

$$
\zeta_{1} \zeta_{2} \ldots \zeta_{n-1}=(-1)^{n-1} \frac{a_{n-1, \gamma}^{(1)}}{a_{0, \gamma}^{(1)}},
$$

onde $\zeta_{i}, i=1, \ldots, n-1$, são os zeros de $P_{1, \gamma}^{*}(z)$, tem-se

$$
\left|\zeta_{1} \zeta_{2} \ldots \zeta_{n-1}\right|=\left|(-1)^{n-1} \frac{a_{n-1, \gamma}^{(1)}}{a_{0, \gamma}^{(1)}}\right|,
$$

ou seja,

$$
\left|\zeta_{1}\right| \cdot\left|\zeta_{2}\right| \ldots\left|\zeta_{n-1}\right|>1
$$

de onde é possível concluir que pelo menos um dos zeros de $P_{1, \gamma}^{*}(z)$ encontra-se fora do disco unitário.

Portanto, os zeros de $P_{\gamma}(z)$ encontram-se no disco unitário quando $\gamma \geq a_{n-1}-2 a_{n}$.

Com isso, demonstrou-se o resultado: 
Proposição 2.2. Seja $P(z)$ um polinômio reflexivo que possui seus zeros no disco unitário cujos coeficientes satisfazem

$$
0<a_{0}<a_{1}=a_{2}=\ldots=a_{n-1} \text { e } a_{n-1}>a_{n}
$$

Os zeros de $P_{\gamma}(z)=P(z)+\gamma z^{n}$ encontram-se no disco unitário quando $\gamma \geq a_{n-1}-2 a_{n}$.

A seguir são apresentados alguns exemplos que ilustram os resultados obtidos.

Exemplo 2.1. Seja

$$
P(z)=z^{3}+3 z^{2}+3 z+1,
$$

cujos zeros encontram-se no disco unitário $(z=-1)$. O polinômio

$$
P_{\gamma}(z)=(1+\gamma) z^{3}+3 z^{2}+3 z+1
$$

possui todos os seus zeros no disco quando $\gamma \geq a_{n-1}-2 a_{n}=1$, como é possível observar na Figura 2.1.

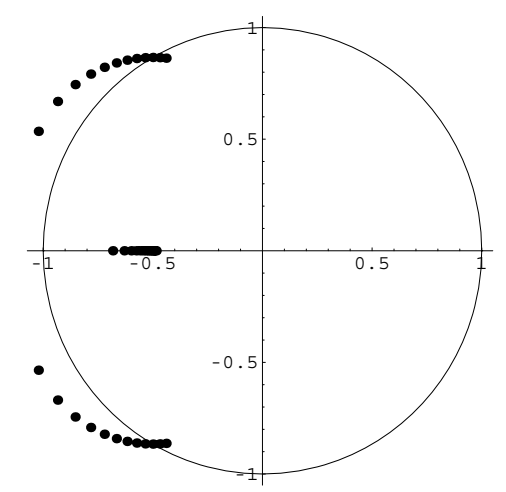

Figura 2.1: Comportamento dos zeros de $P_{\gamma}(z)=(1+\gamma) z^{3}+3 z^{2}+3 z+1,0 \leq \gamma \leq 1.2$.

Nesse caso, o polinômio $P_{\gamma}(z)=(1+\gamma) z^{3}+3 z^{2}+3 z+1$, para $\gamma=0.1,0.2, \ldots, 1.2$, possui um zero real e dois complexos. Quando $\gamma=1$, os zeros complexos encontram-se sobre o círculo unitário e à medida que $\gamma$ aumenta, os zeros de $P_{\gamma}(z)$ entram no círculo.

Exemplo 2.2. Seja

$$
P(z)=z^{4}+2 z^{3}+2 z^{2}+2 z+1
$$

cujos zeros encontram-se no disco unitário. O polinômio

$$
P_{\gamma}(z)=(1+\gamma) z^{4}+2 z^{3}+2 z^{2}+2 z+1
$$

possui todos os seus zeros no disco quando $\gamma \geq 0$, como está ilustrado na Figura 2.2, cujos pontos representam os zeros de $P_{\gamma}(z)=(1+\gamma) z^{4}+2 z^{3}+2 z^{2}+2 z+1$ para $\gamma=0,0.1,0.2, \ldots, 1$. 


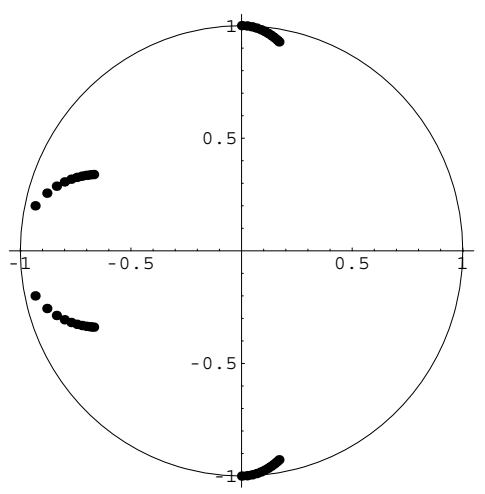

Figura 2.2: Comportamento dos zeros de $P_{\gamma}(z)=(1+\gamma) z^{4}+2 z^{3}+2 z^{2}+2 z+1,0 \leq \gamma \leq 1$.

Exemplo 2.3. Seja

$$
P(z)=2.2 z^{3}+6 z^{2}+6 z+2.2
$$

cujos zeros encontram-se no disco unitário. O polinômio

$$
P_{\gamma}(z)=(2.2+\gamma) z^{3}+6 z^{2}+6 z+2.2
$$

possui todos os seus zeros no disco quando $\gamma \geq 1.6$, como pode ser visto na figura a seguir, onde os pontos representam os zeros de $P_{\gamma}(z)=(2.2+\gamma) z^{3}+6 z^{2}+6 z+2.2$ para $\gamma=0,0.2,0.4, \ldots, 2$.

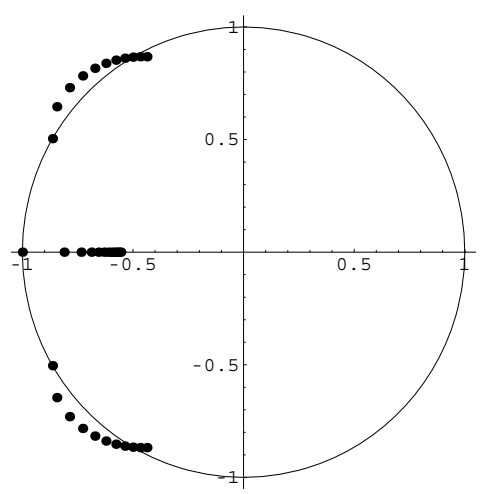

Figura 2.3: Comportamento dos zeros de $P_{\gamma}(z)=(2.2+\gamma) z^{3}+6 z^{2}+6 z+2.2,0 \leq \gamma \leq 2$.

Exemplo 2.4. Seja

$$
P(z)=z^{3}+2 z^{2}+2 z+1,
$$

cujos zeros encontram-se no disco unitário. O polinômio

$$
P_{\gamma}(z)=(1+\gamma) z^{3}+2 z^{2}+2 z+1
$$

possui todos os seus zeros no disco quando $\gamma \geq 0$, como é possível observar na Figura 2.4. 


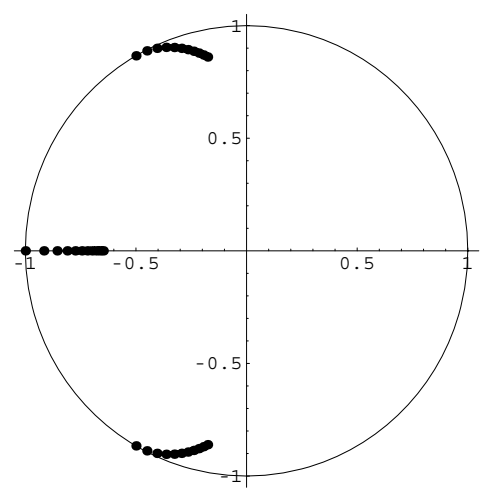

Figura 2.4: Comportamento dos zeros de $P_{\gamma}(z)=(1+\gamma) z^{3}+2 z^{2}+2 z+1,0 \leq \gamma \leq 1$.

Exemplo 2.5. Seja

$$
P(z)=1.5 z^{3}+2 z^{2}+2 z+1.5
$$

cujos zeros encontram-se no disco unitário. O polinômio

$$
P_{\gamma}(z)=(1.5+\gamma) z^{3}+2 z^{2}+2 z+1.5
$$

também possui todos os seus zeros no disco, para $\gamma>0$, pois $P^{\prime}(z)$ tem seus coeficientes ordenados. A Figura 2.5 apresenta os zeros de $P_{\gamma}(z)$ para $0 \leq \gamma \leq 1$.

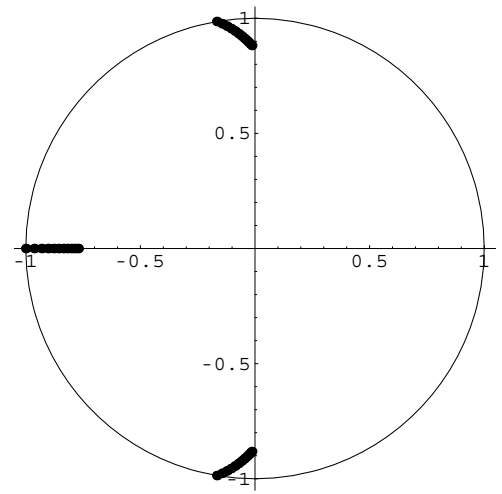

Figura 2.5: Comportamento dos zeros de $P_{\gamma}(z)=(1.5+\gamma) z^{3}+2 z^{2}+2 z+1.5,0 \leq \gamma \leq 1$.

Através dessa abordagem, existem fortes indícios da validade da Conjectura 1.2, pois como já foi mencionado anteriormente, este caso seria um caso crítico da conjectura, sendo que a perturbação no coeficiente dominante faz com que os zeros de $P_{\gamma}(z)$ entrem no disco unitário.

\subsubsection{Caso geral}

Uma das hipóteses da Conjectura 1.2 é que os coeficientes da derivada de $P(z)$ estão ordenados. Então,

$$
2 a_{n}-a_{n-1} \geq 0
$$


sendo $n>1$.

Portanto,

$$
a_{n-1}-2 a_{n} \leq 0 \text {. }
$$

Outra hipótese da Conjectura 1.2 é que os zeros de $P(z)$ encontram-se no disco unitário. Então, através das fórmulas de Vieta, segue que

$$
\left|z_{1} z_{2} \ldots z_{n}\right|=\left|z_{1}\right|\left|z_{2}\right| \ldots\left|z_{n}\right| \leq 1 \Rightarrow \frac{a_{0}}{a_{n}} \leq 1 \Rightarrow a_{0} \leq a_{n} \Rightarrow a_{0}<a_{n}+\gamma
$$

onde $\gamma>0$.

Suponha que $2 a_{0}<a_{1}$ e serão analisados os coeficientes $a_{j}$ em (2.1.1).

Para $j=1$, tem-se:

$$
\begin{array}{ccc}
a_{0}^{(1)} & = & a_{0}^{2}-a_{n}^{2} \\
a_{1}^{(1)} & = & a_{0} a_{1}-a_{n} a_{n-1} \\
a_{2}^{(1)} & = & a_{0} a_{2}-a_{n} a_{n-2} \\
\vdots & & \\
a_{n-1}^{(1)} & = & a_{0} a_{n-1}-a_{n} a_{1} .
\end{array}
$$

Observe que $a_{n-1}^{(1)}<0$, pois

$$
a_{n-1}^{(1)}=a_{0} a_{n-1}-a_{n} a_{1}<2 a_{0} a_{n}-a_{1} a_{n}<0 .
$$

Além disso,

$$
\begin{gathered}
a_{1}^{(1)}-a_{2}^{(1)}=a_{0}\left(a_{1}-a_{2}\right)+a_{n}\left(a_{n-2}-a_{n-1}\right)<0 \\
a_{2}^{(1)}-a_{3}^{(1)}=a_{0}\left(a_{2}-a_{3}\right)+a_{n}\left(a_{n-3}-a_{n-2}\right)<0 \\
\vdots \\
a_{n-2}^{(1)}-a_{n-1}^{(1)}=a_{0}\left(a_{n-2}-a_{n-1}\right)+a_{n}\left(a_{1}-a_{2}\right)<0 .
\end{gathered}
$$

Então,

$$
a_{1}^{(1)}<a_{2}^{(1)}<\ldots<a_{n-1}^{(1)}<0 .
$$

Logo,

$$
\left|a_{1}^{(1)}\right|>\left|a_{2}^{(1)}\right|>\ldots>\left|a_{n-1}^{(1)}\right| .
$$

De maneira análoga dos coeficientes de $P_{1}^{*}(z)$, segue que

$$
\left|a_{1, \gamma}^{(1)}\right|>\left|a_{2, \gamma}^{(1)}\right|>\ldots>\left|a_{n-1, \gamma}^{(1)}\right|
$$

É utilizado o Teorema 2.5 para analisar o comportamento dos zeros de $P_{\gamma}(z)$, pois como $a_{0}<a_{n}+\gamma$, tal teorema pode ser aplicado. 
Sejam

$$
\begin{gathered}
P_{1}^{*}(z)=a_{n-1}^{(1)}+a_{n-2}^{(1)} z+\ldots+a_{0}^{(1)} z^{n-1} \\
P_{1, \gamma}^{*}(z)=a_{n-1, \gamma}^{(1)}+a_{n-2, \gamma}^{(1)} z+\ldots+a_{0, \gamma}^{(1)} z^{n-1} .
\end{gathered}
$$

Sabe-se que as relações (2.2.2) e (2.2.3) são válidas. Resta analisar a relação entre os coeficientes $a_{1}^{(1)}, a_{0}^{(1)}, a_{1, \gamma}^{(1)}$ e $a_{0, \gamma}^{(1)}$.

Considere os seguintes casos:

1. $\left|a_{1}^{(1)}\right| \leq\left|a_{0}^{(1)}\right| \mathrm{e}\left|a_{1, \gamma}^{(1)}\right| \leq\left|a_{0, \gamma}^{(1)}\right|$ :

Como $\left|a_{1}^{(1)}\right| \leq\left|a_{0}^{(1)}\right|$, então $a_{1}^{(1)}-a_{0}^{(1)} \geq 0$.

Assim,

$$
\begin{aligned}
\left|a_{1, \gamma}^{(1)}\right| \leq\left|a_{0, \gamma}^{(1)}\right| & \Leftrightarrow a_{1, \gamma}^{(1)}-a_{0, \gamma}^{(1)} \geq 0 \Leftrightarrow \gamma^{2}+\left(2 a_{n}-a_{n-1}\right) \gamma+a_{1}^{(1)}-a_{0}^{(1)} \geq 0 \\
& \Leftrightarrow \gamma\left(\gamma+2 a_{n}-a_{n-1}\right) \geq 0 \Leftrightarrow \gamma \geq a_{n-1}-2 a_{n} .
\end{aligned}
$$

Logo, como $\gamma>0$ e a desigualdade (2.2.1) é válida, segue que esse caso é satisfeito para qualquer $\gamma$, o que já era de se esperar, pois os coeficientes de $P_{1}^{*}(z)$ estão ordenados.

Por exemplo, considere o polinômio

$$
P(z)=0.9+2 z+2.4 z^{2}+2 z^{3}
$$

que satisfaz as condições da Conjectura 1.2. O polinômio

$$
P_{1}^{*}(z)=-1.84-3 z-3.19 z^{2}
$$

possui seus coeficientes ordenados. Então, $P_{\gamma}(z)=P(z)+\gamma z^{3}$ tem também todos os seus zeros no disco unitário, para $\gamma>0$.

Observe que a condição $\gamma \geq a_{n-1}-2 a_{n}$ é a mesma encontrada no caso do polinômio $P(z)$ ser reflexivo, que foi um caso analisado na subseção anterior.

2. $\left|a_{1}^{(1)}\right|<\left|a_{0}^{(1)}\right| \mathrm{e}\left|a_{1, \gamma}^{(1)}\right|>\left|a_{0, \gamma}^{(1)}\right|$ :

Como $\left|a_{1}^{(1)}\right|<\left|a_{0}^{(1)}\right|$, então $a_{1}^{(1)}-a_{0}^{(1)}>0$. Logo,

$$
\left|a_{1, \gamma}^{(1)}\right|>\left|a_{0, \gamma}^{(1)}\right| \Leftrightarrow a_{1, \gamma}^{(1)}-a_{0, \gamma}^{(1)}<0 \Leftrightarrow \gamma^{2}+\left(2 a_{n}-a_{n-1}\right) \gamma+a_{1}^{(1)}-a_{0}^{(1)}<0,
$$

que não possui solução para $\gamma>0$, pois todos os coeficientes são positivos.

Portanto, esse caso é impossível de ocorrer.

3. $\left|a_{1}^{(1)}\right| \geq\left|a_{0}^{(1)}\right|$ e $\left|a_{1, \gamma}^{(1)}\right| \leq\left|a_{0, \gamma}^{(1)}\right|$ : 
Nesse caso, os coeficientes de $P_{1, \gamma}^{*}(z)$ estão ordenados e assim, pelo Teorema de Eneström-

Kakeya, segue que os zeros de $P_{1, \gamma}^{*}(z)$ encontram-se no disco unitário e, conseqüentemente, os zeros de $P_{\gamma}(z)$ também estão em $|z| \leq 1$.

4. $\left|a_{1}^{(1)}\right|>\left|a_{0}^{(1)}\right|$ e $\left|a_{1, \gamma}^{(1)}\right|>\left|a_{0, \gamma}^{(1)}\right|$ :

Esse caso ainda está sendo pesquisado.

É possível observar, através de testes numéricos, que os coeficientes dos polinômios $P_{j, \gamma}^{*}(z)$ têm as mesmas características dos coeficientes de $P(z)$ e $P_{\gamma}(z)$, ou seja,

$$
\left|a_{1, \gamma}^{(j)}\right|>\left|a_{2, \gamma}^{(j)}\right|>\ldots>\left|a_{n-j, \gamma}^{(j)}\right| \text { e }\left|a_{0, \gamma}^{(j)}\right|<\left|a_{1, \gamma}^{(j)}\right|
$$

Então, o problema se resumiria a determinar a raiz de $P_{n-1, \gamma}^{*}(z)=a_{1, \gamma}^{(n-1)}+a_{0, \gamma}^{(n-1)} z=0$, que tudo indica ter módulo menor ou igual a um.

Para polinômios de grau menor ou igual a três, fica fácil mostrar que a conjectura é válida.

De fato, para $n=1$, o resultado é óbvio, pois a única raiz de $P_{\gamma}(z)=0$ é

$$
z=-\frac{a_{0}}{a_{1}+\gamma}
$$

Para $n=2$, é necessário analisar o que ocorre com a raiz de $P_{1, \gamma}^{*}(z)=a_{1, \gamma}^{(1)}+a_{0, \gamma}^{(1)} z=0$, de acordo com o Teorema 2.5. Observe primeiramente que, como $P(z)$ tem seus zeros no disco, $P_{1}^{*}(z)$ também tem, e então $\left|a_{0}^{(1)}\right| \geq\left|a_{1}^{(1)}\right|$. Mas

$$
\left|a_{0, \gamma}^{(1)}\right|-\left|a_{1, \gamma}^{(1)}\right|=\gamma^{2}+\left(2 a_{2}-a_{1}\right) \gamma+\left|a_{0}^{(1)}\right|-\left|a_{1}^{(1)}\right|>0
$$

para $\gamma>0$

Portanto, $P_{1, \gamma}^{*}(z)$ tem seu zero no disco e, pelo Teorema $2.5, P_{\gamma}(z)$ também tem.

Para $n=3$, é preciso analisar o que ocorre com a raiz de $P_{2, \gamma}^{*}(z)=a_{1, \gamma}^{(2)}+a_{0, \gamma}^{(2)} z=0$.

Observe que

$$
\left|a_{0, \gamma}^{(2)}\right|-\left|a_{1, \gamma}^{(2)}\right|=\left|a_{0, \gamma}^{(1)}-a_{2, \gamma}^{(1)}\right|\left(\left|a_{0, \gamma}^{(1)}+a_{2, \gamma}^{(1)}\right|-\left|a_{1, \gamma}^{(1)}\right|\right) .
$$

Mas

$$
\left|a_{0, \gamma}^{(1)}+a_{2, \gamma}^{(1)}\right|-\left|a_{1, \gamma}^{(1)}\right|=-\left(a_{0}+a_{3}+\gamma\right)(P(-1)-\gamma) \geq 0 .
$$

Logo,

$$
\left|a_{0, \gamma}^{(2)}\right| \geq\left|a_{1, \gamma}^{(2)}\right|
$$


Portanto, $P_{2, \gamma}^{*}(z)$ tem seu zero no disco unitário e, pelo Teorema 2.5, $P_{\gamma}(z)$ também tem.

Para polinômios de grau maior que três, um dos problemas que surge é somente em relação ao sinal dos coeficientes de $P_{j, \gamma}^{*}(z), j=2, . . n-1$. Se for possível mostrar que todos são positivos, não existiria dificuldade em provar a Conjectura 1.2, pois, neste caso, sabe-se que

$$
\left|a_{1, \gamma}^{(j)}\right|>\left|a_{2, \gamma}^{(j)}\right|>\ldots>\left|a_{n-j, \gamma}^{(j)}\right|
$$

para $j=1, \ldots, n-j, \mathrm{e}$

$$
\left|a_{0, \gamma}^{(n-1)}\right|>\left|a_{1, \gamma}^{(n-1)}\right|
$$

Portanto, um desafio agora é mostrar que os coeficientes de $P_{j, \gamma}^{*}(z), j=2, . . n-1$, são todos positivos.

A próxima subseção traz uma análise que inclui o último caso apresentado, sendo possível provar a validade da Conjectura 1.2 quando os coeficientes de $P(z)$ satisfazem algumas condições adicionais, sendo o caso geral objeto de estudos futuros.

\subsubsection{Condições adicionais}

A análise apresentada nesta seção está baseada no Teorema 2.5, pois se $\left|a_{0}\right|<\left|a_{n}\right|, P(z)$ tem todos os seus zeros no disco unitário se, e somente se, o mesmo ocorre com $P_{1}^{*}(z)$. Caso não seja conhecido o comportamento dos zeros de $P_{1}^{*}(z)$, é possível usar o mesmo resultado para analisar os zeros de $P_{2}^{*}(z)$, ou seja, se $\left|a_{n-1}^{(1)}\right|<\left|a_{0}^{(1)}\right|, P_{1}^{*}(z)$ tem todos os seus zeros no disco unitário se, e somente se, $P_{2}^{*}(z)$ também tem. Continuando esse raciocínio, é preciso analisar o que ocorre com o zero do polinômio $P_{n-1}^{*}(z)=a_{1}^{(n-1)}+a_{0}^{(n-1)} z$.

Considere o polinômio $P(z)$, cujos zeros encontram-se no disco unitário e seus coeficientes satisfazem

$$
a_{0}<a_{1}<\ldots<a_{n-1}>a_{n}
$$

e

$$
p a_{i}<(p-1) a_{i+1}, i=0,1, \ldots, n-2, \text { e } p a_{n}>(p-1) a_{n-1},
$$

onde $p$ é um número inteiro positivo, $p>1$.

Sejam os polinômios

$$
P_{j}^{*}(z)=a_{n-j}^{(j)}+a_{n-j-1}^{(j)} z+\ldots+a_{0}^{(j)} z^{n-j}
$$

$\mathrm{e}$

$$
P_{j, \gamma}^{*}(z)=a_{n-j, \gamma}^{(j)}+a_{n-j-1, \gamma}^{(j)} z+\ldots+a_{0, \gamma}^{(j)} z^{n-j},
$$

relacionados, respectivamente, aos polinômios $P(z)$ e $P_{\gamma}(z)$, cujos coeficientes são obtidos da expressão (2.1.1). 
Como o polinômio $P(z)$ tem todos os seus zeros no disco unitário (por hipótese da Conjectura 1.2), segue, pelo Teorema 2.5, que todos os polinômios $P_{j}^{*}(z)$ também têm.

Resta analisar o que ocorre com os zeros dos polinômios $P_{j, \gamma}^{*}(z)$.

Já foi visto que, para $j=1$,

$$
a_{1}^{(1)}<a_{2}^{(1)}<\ldots<a_{n-1}^{(1)} .
$$

Como $a_{n-1}^{(1)}<0$, pois

$$
a_{n-1}^{(1)}=a_{0} a_{n-1}-a_{n} a_{1}<\frac{p-1}{p} a_{1} a_{n-1}-a_{n} a_{1}=a_{1}\left(\frac{p-1}{p} a_{n-1}-a_{n}\right)<0,
$$

então, de (2.2.4) segue que $a_{i}^{(1)}<0$ para $i=1,2, \ldots, n-1$.

Portanto,

$$
\left|a_{1}^{(1)}\right|>\left|a_{2}^{(1)}\right|>\ldots>\left|a_{n-1}^{(1)}\right| .
$$

Da mesma forma que os coeficientes de $P(z)$, os coeficientes de $P_{1}^{*}(z)$ satisfazem

$$
p\left|a_{n-i}^{(1)}\right|<(p-1)\left|a_{n-i-1}^{(1)}\right|, \text { para } i=1,2, \ldots, n-2,
$$

$\mathrm{e}$

$$
p\left|a_{0}^{(1)}\right|>(p-1)\left|a_{1}^{(1)}\right|
$$

De fato,

$$
\begin{aligned}
p a_{0}^{(1)}-(p-1) a_{1}^{(1)} & =p\left(a_{0}^{2}-a_{n}^{2}\right)-(p-1)\left(a_{0} a_{1}-a_{n} a_{n-1}\right) \\
& =a_{0}\left(p a_{0}-(p-1) a_{1}\right)+a_{n}\left((p-1) a_{n-1}-p a_{n}\right)<0 .
\end{aligned}
$$

Então,

$$
p a_{0}^{(1)}-(p-1) a_{1}^{(1)}<0 \Rightarrow p a_{0}^{(1)}<(p-1) a_{1}^{(1)}<0 \Rightarrow a_{0}^{(1)}<0 .
$$

Portanto,

$$
p\left|a_{0}^{(1)}\right|>(p-1)\left|a_{1}^{(1)}\right|
$$

Além disso, para $i=1,2, \ldots, n-2$, segue que

$$
\begin{aligned}
p a_{n-i}^{(1)}-(p-1) a_{n-i-1}^{(1)} & =p\left(a_{0} a_{n-i}-a_{n} a_{i}\right)-(p-1)\left(a_{0} a_{n-i-1}-a_{n} a_{i+1}\right) \\
& =a_{0}\left(p a_{n-i}-(p-1) a_{n-i-1}\right)+a_{n}\left((p-1) a_{i+1}-p a_{i}\right)>0 .
\end{aligned}
$$


Portanto, para $i=1,2, \ldots, n-2$,

$$
p\left|a_{n-i}^{(1)}\right|<(p-1)\left|a_{n-i-1}^{(1)}\right| .
$$

De maneira análoga é possível provar que o mesmo ocorre com os coeficientes do polinômio $P_{1, \gamma}^{*}(z)$.

Para $j=2$,

$$
a_{1}^{(2)}>a_{2}^{(2)}>\ldots>a_{n-2}^{(2)}
$$

Como $a_{n-2}^{(2)}>0$, pois

$$
a_{n-2}^{(2)}=a_{0}^{(1)} a_{n-2}^{(1)}-a_{n-1}^{(1)} a_{1}^{(1)}>\frac{(p-1)}{p} a_{1}^{(1)} a_{n-2}^{(1)}-a_{n-1}^{(1)} a_{1}^{(1)}=a_{1}^{(1)}\left(\frac{p-1}{p} a_{n-2}^{(1)}-a_{n-1}^{(1)}\right)>0
$$

então, de (2.2.5) segue que $a_{i}^{(2)}>0$ para $i=1,2, \ldots, n-2$.

Portanto,

$$
a_{1}^{(2)}>a_{2}^{(2)}>\ldots>a_{n-2}^{(2)}
$$

Da mesma forma que os coeficientes de $P_{1}^{*}(z)$, os coeficientes de $P_{2}^{*}(z)$ satisfazem

$$
p a_{n-i}^{(2)}<(p-1) a_{n-i-1}^{(2)}, \text { para } i=2, \ldots, n-2,
$$

e

$$
p\left|a_{0}^{(2)}\right|>(p-1)\left|a_{1}^{(2)}\right|
$$

De fato,

$$
\begin{aligned}
p a_{0}^{(2)}-(p-1) a_{2}^{(1)} & =p\left(\left(a_{0}^{(1)}\right)^{2}-\left(a_{n-1}^{(1)}\right)^{2}\right)-(p-1)\left(a_{0}^{(1)} a_{1}^{(1)}-a_{n-2}^{(1)} a_{n-1}^{(1)}\right) \\
& =a_{0}^{(1)}\left(p a_{0}^{(1)}-(p-1) a_{1}^{(1)}\right)+a_{n-1}^{(1)}\left((p-1) a_{n-2}^{(1)}-p a_{n-1}^{(1)}\right)>0 .
\end{aligned}
$$

Então,

$$
p a_{0}^{(2)}-(p-1) a_{1}^{(2)}>0 \Rightarrow p a_{0}^{(2)}>(p-1) a_{1}^{(2)}>0 \Rightarrow a_{0}^{(2)}>0 .
$$

Portanto,

$$
p a_{0}^{(2)}>(p-1) a_{1}^{(2)}
$$

Além disso, para $i=2, \ldots, n-2$,

$$
\begin{aligned}
p a_{n-i}^{(2)}-(p-1) a_{n-i-1}^{(2)} & =p\left(a_{0}^{(1)} a_{n-i}^{(1)}-a_{n-1}^{(1)} a_{i-1}^{(1)}\right)-(p-1)\left(a_{0}^{(1)} a_{n-i-1}^{(1)}-a_{n-1}^{(1)} a_{i}^{(1)}\right) \\
& =a_{0}^{(1)}\left(p a_{n-i}^{(1)}-(p-1) a_{n-i-1}^{(1)}\right)+a_{n}^{(1)}\left((p-1) a_{i+1}^{(1)}-p a_{i}^{(1)}\right)>0 .
\end{aligned}
$$


Portanto, para $i=2, \ldots, n-2$,

$$
p\left|a_{n-i}^{(1)}\right|<(p-1)\left|a_{n-i-1}^{(1)}\right| .
$$

De maneira análoga prova-se que o mesmo ocorre com os coeficientes do polinômio $P_{2, \gamma}^{*}(z)$. Como hipótese de indução, suponha que, para $j$ qualquer,

$$
\begin{gathered}
a_{i}^{(j)}>0, \text { para } j=0,1, \ldots, n-j, a_{1}^{(j)}>a_{2}^{(j)}>\ldots>a_{n-j}^{(j)}, \\
p a_{0}^{(j)}>(p-1) a_{1}^{(j)} \text { e } p a_{n-i}^{(j)}<(p-1) a_{n-i-1}^{(j)} \text {, para } i=j, \ldots, n-2 .
\end{gathered}
$$

A seguir é verificado o que ocorre para $j+1(j=2, \ldots, n-3)$.

Para $i=1, . ., n-j$,

$$
\begin{aligned}
a_{i}^{(j+1)}-a_{i+1}^{(j+1)} & =a_{0}^{(j)} a_{i}^{(j)}-a_{n-j}^{(j)} a_{n-j-i}^{(j)}-a_{0}^{(j)} a_{i+1}^{(j)}+a_{n-j}^{(j)} a_{n-j-i-1}^{(j)} \\
& =a_{0}^{(j)}\left(a_{i}^{(j)}-a_{i+1}^{(j)}\right)+a_{n-j}^{(j)}\left(a_{n-j-i-1}^{(j)}-a_{n-j-i}^{(j)}\right)>0 .
\end{aligned}
$$

Observe também que

$$
\begin{aligned}
a_{n-(j+1)}^{(j+1)} & =a_{0}^{(j)} a_{n-(j+1)}^{(j)}-a_{n-j}^{(j)} a_{1}^{(j)}>\frac{p-1}{p} a_{1}^{(j)} a_{n-(j+1)}^{(j)}-a_{n-j}^{(j)} a_{1}^{(j)} \\
& >a_{1}^{(j)}\left(\frac{p-1}{p} a_{n-(j+1)}^{(j)}-a_{n-j}^{(j)}\right)>0 .
\end{aligned}
$$

Portanto,

$$
a_{1}^{(j+1)}>a_{2}^{(j+1)}>\ldots>a_{n-(j+1)}^{(j+1)}>0 .
$$

A desigualdade $p a_{0}^{(j+1)}>(p-1) a_{1}^{(j+1)}$ é verdadeira, pois

$$
\begin{aligned}
p a_{0}^{(j+1)}-(p-1) a_{1}^{(j+1)} & =p\left(\left(a_{0}^{(j)}\right)^{2}-\left(a_{n-j}^{(j)}\right)^{2}\right)-(p-1)\left(a_{0}^{(j)} a_{1}^{(j)}-a_{n-j}^{(j)} a_{n-j-1}^{(j)}\right) \\
& =a_{0}^{(j)}\left(p a_{0}^{(j)}-(p-1) a_{1}^{(j)}\right)+a_{n-j}^{(j)}\left((p-1) a_{n-j-1}^{(j)}-p a_{n-j}^{(j)}\right)>0 .
\end{aligned}
$$

Então, da desigualdade acima é possível concluir que $a_{0}^{(j+1)}>0$.

Além disso, para $i=j+1, \ldots, n-2$,

$$
\begin{aligned}
p a_{n-i}^{(j+1)}-(p-1) a_{n-i-1}^{(j+1)} & =p\left(a_{0}^{(j)} a_{n-i}^{(j)}-a_{n-j}^{(j)} a_{i-j}^{(j)}\right)-(p-1)\left(a_{0}^{(j)} a_{n-i-1}^{(j)}-a_{n-j}^{(j)} a_{i-j+1}^{(j)}\right) \\
& =a_{0}^{(j)}\left(p a_{n-i}^{(j)}-(p-1) a_{n-i-1}^{(j)}\right)+a_{n-j}^{(j)}\left((p-1) a_{i-j+1}^{(j)}-p a_{i-j}\right)<0 .
\end{aligned}
$$


Portanto, para $i=j+1, \ldots, n-2$,

$$
p a_{n-i}^{(j+1)}<(p-1) a_{n-i-1}^{(j+1)}
$$

De maneira análoga prova-se que o mesmo ocorre com os coeficientes do polinômio $P_{j+1, \gamma}^{*}(z)$. Resta analisar o polinômio $P_{n-1, \gamma}^{*}(z)=a_{1, \gamma}^{(n-1)}+a_{0, \gamma}^{(n-1)} z$.

Observe que

$$
\begin{aligned}
a_{1}^{(n-1)} & =a_{0}^{(n-2)} a_{1}^{(n-2)}-a_{1}^{(n-2)} a_{2}^{(n-2)}>\frac{p-1}{p} a_{1}^{(n-2)} a_{1}^{(n-2)}-a_{1}^{(n-2)} a_{2}^{(n-2)} \\
& =a_{1}^{(n-2)}\left(\frac{p-1}{p} a_{1}^{(n-2)}-a_{2}^{(n-2)}\right)>0 .
\end{aligned}
$$

Portanto, $a_{1}^{(n-1)}>0$.

Além disso,

$$
\begin{aligned}
p a_{0}^{(n-1)}-(p-1) a_{1}^{(n-1)} & =p\left(\left(a_{0}^{(n-2)}\right)^{2}-\left(a_{2}^{(n-2)}\right)^{2}\right)-(p-1)\left(a_{0}^{(n-2)} a_{1}^{(n-2)}-a_{1}^{(n-2)} a_{2}^{(n-2)}\right) \\
& =a_{0}^{(n-2)}\left(p a_{0}^{(n-2)}-(p-1) a_{1}^{(n-2)}\right)+a_{2}^{(n-2)}\left((p-1) a_{1}^{(n-2)}-p a_{2}^{(n-2)}\right) \\
& >0 .
\end{aligned}
$$

Logo, $a_{0}^{(n-1)}>0$.

Para analisar o que ocorre $\operatorname{com} a_{0}^{(n-1)}-a_{1}^{(n-1)}$, observe que

$$
\begin{aligned}
a_{0}^{(n-1)}-a_{1}^{(n-1)}= & \left(a_{0}^{(n-2)}-a_{2}^{(n-2)}\right)\left(a_{0}^{(n-3)}+a_{3}^{(n-3)}\right) \ldots\left(a_{0}^{(1)}+(-1)^{n} a_{n-1}^{(1)}\right) \\
& \left(a_{0}+(-1)^{n+1} a_{n}\right)\left((-1)^{n} a_{n}+(-1)^{n-1} a_{n-1}+\ldots+a_{0}\right) .
\end{aligned}
$$

Já foi visto que, para $j=2,3, \ldots, n-1$,

$$
0<a_{0}^{(j)}=\left(a_{0}^{(j-1)}\right)^{2}-\left(a_{n-(j-1)}^{(j-1)}\right)^{2}=\left(a_{0}^{(j-1)}-a_{n-(j-1)}^{(j-1)}\right)\left(a_{0}^{(j-1)}+a_{n-(j-1)}^{(j-1)}\right) .
$$

Então, $a_{0}^{(j-1)}-a_{n-(j-1)}^{(j-1)}>0$ e $a_{0}^{(j-1)}+a_{n-(j-1)}^{(j-1)}>0$.

Em (2.2.6), é possível observar que $(-1)^{n} a_{n}+(-1)^{n-1} a_{n-1}+\ldots+a_{0}=P(-1)$.

Além disso, $P(-1) \geq 0$ para $n$ par e $P(-1) \leq 0$ para $n$ ímpar.

Então, de (2.2.6),

$$
a_{0}^{(n-1)}-a_{1}^{(n-1)} \geq 0
$$

Portanto, $P_{n-1}^{*}(z)$ tem seu zero no disco unitário.

No caso do polinômio $P_{n-1, \gamma}(z)$, a mesma demonstração é usada para provar que $a_{0, \gamma}^{(n-1)}>0$ e $a_{1, \gamma}^{(n-1)}>0$. 
Além disso,

$$
\begin{aligned}
a_{0, \gamma}^{(n-1)}-a_{1, \gamma}^{(n-1)}= & \left(a_{0, \gamma}^{(n-2)}-a_{2, \gamma}^{(n-2)}\right)\left(a_{0, \gamma}^{(n-3)}+a_{3, \gamma}^{(n-3)}\right) \ldots\left(a_{0, \gamma}^{(1)}+(-1)^{n} a_{n-1, \gamma}^{(1)}\right) \\
& \left(a_{0}+(-1)^{n+1}\left(a_{n}+\gamma\right)\right)\left((-1)^{n}\left(a_{n}+\gamma\right)+(-1)^{n-1} a_{n-1}+\ldots+a_{0}\right) \\
= & \left(a_{0, \gamma}^{(n-2)}-a_{2, \gamma}^{(n-2)}\right)\left(a_{0, \gamma}^{(n-3)}+a_{3, \gamma}^{(n-3)}\right) \ldots\left(a_{0, \gamma}^{(1)}+(-1)^{n} a_{n-1, \gamma}^{(1)}\right) \\
& \left(a_{0}+(-1)^{n+1}\left(a_{n}+\gamma\right)\right)\left((-1)^{n} \gamma+P(-1)\right) \geq 0 .
\end{aligned}
$$

Logo, $a_{0, \gamma}^{(n-1)}-a_{1, \gamma}^{(n-1)}>0$ e $P_{n-1, \gamma}^{*}(z)$ tem seu zero no disco unitário e, como conseqüência do Teorema 2.5, $P_{\gamma}(z)$ também tem.

Portanto, esta é a demonstração do seguinte resultado:

Proposição 2.3. Seja $P(z)=\sum_{i=0}^{n} a_{i} z^{i}$ um polinômio cujos zeros encontram-se no disco unitário e os coeficientes satisfazem

$$
0<a_{0}<a_{1}<\ldots<a_{n-1}>a_{n}, p a_{i}<(p-1) a_{i+1}, i=0,1, \ldots, n-2, e p a_{n}>(p-1) a_{n-1}
$$

onde p é um número inteiro positivo. Então, o polinômio $P_{\gamma}(z)=P(z)+\gamma z^{n}, \gamma>0$, possui todos os seus zeros no disco unitário.

Esse é um dos principais resultados obtidos neste trabalho, pois além de ser inédito, será importante para dar suporte à Conjectura de Jeltsch, pois no caso dos métodos $(K, L)$ de Brown, para $K \leq K_{L}^{*}$, os coeficientes de $\rho(z)$ satisfazem

$$
2 \alpha_{j}<\alpha_{j+1} \text { e } 2 \alpha_{K}>\alpha_{K-1}
$$

como foi citado anteriormente.

Portanto, no caso dos polinômios característicos relacionados aos métodos $(K, L)$ de Brown, segue que a Conjectura de Jeltsch é válida para $K \leq K_{L}^{*}$.

No próximo capítulo tem-se maiores detalhes a respeito da Conjectura 1.1.

Os resultados obtidos neste capítulo, além de serem importantes na análise da estabilidade dos métodos $(K, L)$ de Brown, são também de fundamental importância na área de zeros de polinômios, pois é possível visualizar a Conjectura 1.2 como uma generalização do Teorema de Eneström-Kakeya, pois a ordenação dos coeficientes é satisfeita, com exceção do coeficiente dominante. 


\section{Métodos $(K, L)$ de Brown}

Neste capítulo são apresentadas algumas propriedades dos métodos $(K, L)$ de Brown e é utilizada a teoria das order stars para analisá-las, como, por exemplo, as questões de estabilidade. Primeiramente são apresentadas propriedades importantes relacionadas aos métodos $(K, L)$ de Brown. A segunda seção traz algumas definições e resultados sobre order stars, pois tal ferramenta será muito utilizada no decorrer deste capítulo. Depois são apresentadas algumas características dos métodos de Brown e são provados alguns resultados importantes através das order stars.

\subsection{Introdução}

Os métodos $(K, L)$ de Brown, introduzidos por Brown (1977), formam uma classe de métodos multiderivadas de passo múltiplo para a solução de equações diferenciais ordinárias. Foram introduzidos como métodos com menor número de passos e melhor precisão para a solução de problemas. Tais métodos possuem boas propriedades de estabilidade, como grandes regiões de estabilidade absoluta. Por esse motivo, os métodos multiderivadas são indicados para resolver problemas stiff.

Como já foi visto anteriormente, os métodos $(K, L)$ de Brown são representados por

$$
\sum_{i=0}^{K} \alpha_{i} y_{n+i}=\sum_{j=1}^{L} \beta_{j} h^{j} f_{n+K}^{(j-1)}
$$


onde as constantes $\alpha_{i}$ e $\beta_{j}$ são escolhidas de modo a obter a máxima precisão. A construção explícita desta classe de métodos pode ser encontrada em Jeltsch e Kratz (1978).

Os coeficientes $\alpha_{i}$ e $\beta_{j}$ são dados por

$$
\begin{aligned}
\alpha_{i} & =(-1)^{K-i}\left(\begin{array}{c}
K \\
i
\end{array}\right)(K-i)^{-L}, i=0, \ldots, K-1, \\
\alpha_{K} & =-\left(\alpha_{0}+\alpha_{1}+\ldots+\alpha_{K-1}\right), \\
\beta_{j} & =\frac{(-1)^{j}}{j !} \sum_{i=0}^{K-1}(-1)^{K-i}\left(\begin{array}{c}
K \\
i
\end{array}\right)(K-i)^{j-L}, j=1, \ldots, L .
\end{aligned}
$$

A seguir são apresentados alguns resultados sobre os métodos $(K, L)$, cujo estudo detalhado pode ser visto em Meneguette (1987).

Teorema 3.1. Os métodos $(K, L)$ de Brown têm ordem de consistência $p=K+L-1$.

Observe que, como $K, L \geq 1$, estes métodos têm ordem pelo menos um. Logo, são sempre consistentes para quaisquer valores de $K$ e $L$.

Se os métodos $(K, L)$ têm ordem $p$, então

$$
\rho\left(e^{z}\right)-\sum_{j=1}^{L} z^{j} \sigma_{j}\left(e^{z}\right)=\sum_{j=p+1}^{\infty} C_{j} z^{j}, C_{p+1} \neq 0
$$

onde $\rho(\xi)$ e $\sigma_{j}(\xi)$ são os polinômios característicos relacionados aos métodos $(K, L)$ de Brown, dados por

$$
\rho(\xi)=\sum_{i=0}^{K} \alpha_{i} \xi^{i}
$$

e

$$
\sigma_{j}(\xi)=\beta_{j} \xi^{K}, j=1,2, \ldots, L
$$

Para analisar a convergência de métodos lineares de passo múltiplo, basta verificar se o método é consistente e zero-estável, sendo este um resultado clássico de Dahlquist. No caso de métodos multiderivadas de passo múltiplo, este resultado também é válido.

Um método é zero-estável quando os zeros do polinômio $\rho(\xi)$ encontram-se no disco unitário e os zeros de módulo um são simples. Além disso, diz-se que um método numérico é fortemente estável quando é zero-estável e tem $z=1$ como a única possibilidade para os zeros de módulo um.

Para analisar a convergência dos métodos $(K, L)$, basta analisar a zero-estabilidade, pois como já foi visto, são sempre consistentes.

Além do conceito de zero-estabilidade, existem outros conceitos de estabilidade, que são: $A$ estabilidade, $A(\alpha)$-estabilidade, $A_{0}$-estabilidade e stiff-estabilidade. Tais conceitos diferem da zero-estabilidade porque o passo $h$ se mantém fixo. 
Existem alguns métodos que são estáveis apenas para alguns valores de $h$. Tais valores são encontrados quando o método linear de passo múltiplo é aplicado à equação teste $y^{\prime}=\lambda y$. A motivação para este tipo de estudo de estabilidade pode ser encontrada em Lambert (1973).

O polinômio característico do método linear de passo múltiplo é dado por

$$
\pi(w, z)=\rho(w)-z \sigma(w), z=h \lambda .
$$

Já o polinômio característico do método multiderivada de passo múltiplo pode ser representado por

$$
\pi(w, z)=\rho(w)-\sum_{j=1}^{L} z^{j} \sigma_{j}(w), z=h \lambda .
$$

Portanto, a estabilidade do método depende dos ramos $w_{i}(z), 1 \leq i \leq k$, da função $w(z)$ definida por $\pi(w, z)=0$. Observe que $\pi(w, z) \rightarrow \rho(w)$ quando $h \rightarrow 0$ e $w_{i}(h) \rightarrow w_{i}, 1 \leq i \leq k$, sendo $\left\{w_{i}\right\}$ os zeros de $\rho(w)$.

Para um método multiderivada consistente, $\rho(1)=0$. Este zero, representado por $w_{1}(h)$, é um ramo de $\pi(w, z)=0$, pois $w_{1}(h) \rightarrow 1$ quando $h \rightarrow 0$. Este ramo recebe o nome de ramo principal de $\pi(w, z)=0$.

Considerando métodos convergentes, sejam $w_{1}(z)$ o ramo principal e $\Gamma$ a maior região na qual $w_{1}(z)$ pode ser analiticamente continuada.

Definição 3.1. Os conjuntos A e R, dados por

$$
\begin{gathered}
A=\left\{h \lambda \in \mathbb{C}|| w_{i}(h \lambda) \mid \leq 1,1 \leq i \leq k\right\} \\
R=\left\{h \lambda \in \Gamma|| w_{i}(h \lambda)|\leq| w_{1}(h \lambda) \mid, 2 \leq i \leq k\right\}
\end{gathered}
$$

definem, respectivamente, as regiões de estabilidades absoluta e relativa do método numérico.

Definição 3.2. A seguir são apresentados outros conceitos de estabilidade:

1. O método numérico é chamado de $A_{0}$-estável se $(-\infty, 0) \subset A$.

2. O método numérico é chamado de A-estável se $\{h \lambda \in \mathbb{C} \mid \operatorname{Re}(h \lambda)<0\} \subset A$.

3. O método numérico é $A(\alpha)$-estável se $W_{\alpha}=\{h \lambda \in \mathbb{C}|| \arg (-h \lambda) \mid<\alpha\} \subset A$.

4. O método numérico é $A(0)$-estável se existe $\alpha>0$ tal que $W_{\alpha} \subset A$.

5. O método numérico é dito stiff-estável se é $A_{0}$-estável com $R_{1} \cup R_{2} \subset A$ e $R_{3} \subset R$, onde

$$
\begin{aligned}
& R_{1}=\{h \lambda \in \mathbb{C} \mid \operatorname{Re}(h \lambda)<-a\} \\
& R_{2}=\{h \lambda \in \mathbb{C}|\operatorname{Re}(h \lambda)<-b,| \operatorname{Im}(h \lambda) \mid<c\} \\
& R_{3}=\{h \lambda \in \mathbb{C}|| \operatorname{Re}(h \lambda)|<b,| \operatorname{Im}(h \lambda) \mid<c\},
\end{aligned}
$$


sendo a, b e c constantes positivas.

A $A$-estabilidade também pode ser estabelecida por meio do resultado abaixo.

Teorema 3.2. Um método multiderivada de passo múltiplo genérico

$$
\sum_{i=0}^{K} \alpha_{i} y_{n+i}=\sum_{i=0}^{K} \sum_{j=1}^{L} h^{j} \beta_{i j} f_{n+i}^{(j-1)}
$$

de ordem $p$ é A-estável somente se $p \leq 2 L$.

A demonstração desse teorema pode ser encontrada em Iserles e Norsett (1991). Portanto, de acordo com o Teorema 3.2, os métodos $(K, L)$ de Brown são $A$-estáveis somente se $K+L-1 \leq 2 L$, ou seja, $K \leq L+1$.

Serão apresentadas, mais adiante, as regiões de estabilidade absoluta para alguns valores fixos de $K$ e $L$ dos métodos $(K, L)$ de Brown, pois não é comum na literatura encontrar tais regiões de estabilidade.

Sabe-se que $\rho(1)=0$. Além disso, este é o único zero de $\rho(z)$ que possui módulo um. Logo, os métodos $(K, L)$ de Brown zero-estáveis são fortemente estáveis. Então, para analisar a zeroestabilidade dos métodos $(K, L)$, pode-se considerar o polinômio

$$
\phi_{0}(z)=\frac{\rho(z)}{z-1}=\sum_{i=0}^{K-1} a_{i 0} z^{i}
$$

onde $a_{i 0}=\sum_{j=i+1}^{K} \alpha_{j}=-\sum_{j=0}^{i} \alpha_{j}, 0 \leq i \leq K-1$.

Mas, analisar os zeros de $\phi_{0}(z)$ é equivalente a analisar os zeros de $\psi(z)=(-1)^{K-1} \phi_{0}(-z)$, pois a quantidade de zeros no disco unitário é a mesma.

De acordo com Jeltsch e Kratz (1978), os coeficientes de $\psi(z)$, para $K \leq 2(L+1)$, satisfazem o Teorema de Eneström-Kakeya e, conseqüentemente, $\psi(z)$ possui $K-1$ zeros no disco unitário. De fato, é possível observar que

$$
\psi(z)=(-1)^{K-1} \phi_{0}(-z)=(-1)^{K-1} \frac{\rho(-z)}{-z-1}=(-1)^{K} \frac{\rho(-z)}{z+1}=q_{0}+q_{1} z+\ldots+q_{n-1} z^{n-1},
$$

onde

$$
\begin{aligned}
q_{0} & =a_{0} \\
q_{1} & =a_{1}-a_{0} \\
& \vdots \\
q_{n-2} & =a_{n-1}-a_{n} \\
q_{n-1} & =a_{n} .
\end{aligned}
$$


Logo,

$$
q_{0}<q_{1}<\ldots<q_{n-1} .
$$

Portanto, o seguinte resultado pode ser estabelecido.

Teorema 3.3. Os métodos $(K, L)$ de Brown são fortemente estáveis para $K \leq 2(L+1)$.

Serão apresentados adiante mais resultados a respeito da zero-estabilidade dos métodos $(K, L)$, sendo utilizada a teoria das order stars para demonstrá-los. A seguir, alguns resultados apresentados por Jeltsch (1979) são enunciados.

Teorema 3.4. Os métodos $(K, L)$ de Brown são stiff-estáveis se, e somente se, são $A_{0}$ e fortemente estáveis.

Teorema 3.5. Os métodos $(K, L)$ de Brown, para L fixo, são stiff-estáveis se $K \leq \frac{3}{2}(L+1)$.

Em Meneguette (1987), foi provada a existência de uma subclasse dos métodos $(K, L)$ de Brown onde os coeficientes do polinômio característico $\rho(z)$ satisfazem:

1. $0<\alpha_{0}<\alpha_{1}<\ldots<\alpha_{K-1}$ mas $\alpha_{K}<\alpha_{K-1}$, para $K \leq K_{L}$;

2. $2 \alpha_{j}<\alpha_{j+1}$ para $0 \leq j \leq K_{L}^{*}$;

onde

$$
K_{L}=\min \left\{2^{L+1}+1, \frac{3^{L+1}}{2^{L}}+2, \ldots, \frac{(L+1)^{L+1}}{L^{L}}+L\right\}
$$

$\mathrm{e}$

$$
K_{L}^{*}=\min \left\{2^{L}+1, \frac{3^{L+1}}{2^{L+1}}+2, \ldots, \frac{(L+1)^{L+1}}{2 L^{L}}+L\right\}
$$

como já foi visto anteriormente. Além disso, para esta classe de métodos, o seguinte resultado é válido:

Teorema 3.6. Os métodos $(K, L)$ de Brown são fortemente estáveis para $K \leq K_{L}$.

Observe que $K_{L}^{*}<K_{L}$.

Já foi visto que os métodos $(K, L)$ de Brown são stiff-estáveis se, e somente se, são $A_{0} \mathrm{e}$ fortemente estáveis.

Um resultado não cogitado por Jeltsch demonstra que $A_{0}$-estabilidade dos métodos $(K, L)$ de Brown está relacionada ao polinômio

$$
\rho_{\gamma}(z)=\rho(z)+\gamma z^{K}
$$

onde $\gamma$ é um parâmetro real variando de $0 \mathrm{a} \infty$, como já foi citado anteriormente.

Se for possível mostrar a validade da Conjectura 1.2, a condição para a determinação de métodos stiff-estáveis, de acordo com o Teorema 3.4, se resumiria a determinar métodos zero-estáveis, 
pois os métodos $(K, L)$ zero-estáveis são fortemente estáveis e se $\rho_{\gamma}(z)$ possuir todos os seus zeros no disco unitário, tais métodos são $A_{0}$-estáveis. Então, todos os métodos $(K, L)$ de Brown são stiff-estáveis se, e somente se, são zero-estáveis.

Conforme mostrado em Meneguette (1987), se $\gamma>\alpha_{K-1}-\alpha_{K}$, todos os zeros de $\rho_{\gamma}(z)$ encontram-se no disco unitário, como conseqüência do Teorema de Eneström-Kakeya. Se $0<\gamma<\alpha_{K-1}-\alpha_{K}$, o comportamento dos zeros ainda não é conhecido.

Conforme analisado no capítulo anterior, no caso em que os coeficientes de $P(z)$ satisfazem algumas condições adicionais, dadas por

$$
p a_{i}<(p-1) a_{i+1}, i=0,1, \ldots, n-2, \text { e } p a_{n}>(p-1) a_{n-1},
$$

onde $p$ é uma constante positiva, $p>1$, a Conjectura 1.2 é válida.

Então, como para $K \leq K_{L}^{*}$ os coeficientes de $\rho(z)$ satisfazem

$$
2 \alpha_{j}<\alpha_{j+1} \text { e } 2 \alpha_{K}>\alpha_{K-1}
$$

segue que a Conjectura 1.2 é verdadeira para $K \leq K_{L}^{*}$. Portanto, a Conjectura 1.1 é válida para $K \leq K_{L}^{*}$, e então, para uma classe de métodos $(K, L)$, a conjectura de Jeltsch se verifica.

A seguir são apresentados alguns resultados e definições básicas sobre order stars, pois adiante será utilizada tal teoria para demonstrar outras propriedades dos métodos $(K, L)$ de Brown.

\subsection{Order stars}

O conceito de order stars foi introduzido por Wanner et al. (1978). A idéia principal das order stars é explorar diferentes características de algoritmos numéricos como propriedades de funções analíticas em várias regiões do plano complexo, ou seja, descrever ordem, estabilidade e suas relações como características de funções complexas.

É, em geral, muito útil para a análise de famílias de métodos e respectivas barreiras a que elas estão sujeitas principalmente em relação à estabilidade, que é o principal foco deste trabalho.

Num dos primeiros artigos sobre order stars, Wanner et al. (1978) analisaram algumas conjecturas utilizando esta nova teoria: a conjectura de Ehle, sobre a $A$-aceitabilidade ( $A$-acceptability) das aproximações de Padé para a função exponencial, que é verdadeira; a conjectura de Norsett, sobre os zeros do "E-polinômio", que é falsa; a conjectura de Daniel e Moore, sobre a obtenção da ordem máxima de certos métodos de passo múltiplo $A$-estáveis, que é verdadeira, generalizando o clássico teorema de Dahlquist.

Portanto, o objetivo desta seção é expor como o conceito das order stars foi utilizado no início do seu desenvolvimento. 
Para isso, considere a aproximação racional $R(z)$ da função exponencial

$$
R(z)=\frac{P_{k}(z)}{Q_{j}(z)}
$$

onde

$$
P_{k}(z)=1+\frac{k}{j+k} z+\frac{k(k-1)}{(j+k)(j+k-1)} \frac{z^{2}}{2 !}+\ldots+\frac{k(k-1) \ldots 1}{(j+k) \ldots(j+1)} \frac{z^{k}}{k !}
$$

$\mathrm{e}$

$$
Q_{j}(z)=1-\frac{j}{k+j} z+\frac{j(j-1)}{(k+j)(k+j-1)} \frac{z^{2}}{2 !}+\ldots+(-1)^{j} \frac{j(j-1) \ldots 1}{(k+j) \ldots(k+1)} \frac{z^{j}}{j !} .
$$

A aproximação $R(z)$ recebe o nome de aproximação de Padé de ordem $k+j$. Ela é muito utilizada na análise da estabilidade de classe de métodos numéricos de ordem 1, pois algumas aproximações racionais da função exponencial, como $R(z)$, são limitadas por 1 na região do plano complexo onde $\operatorname{Re}(z) \leq 0$. Como exemplo,

1. para $k=1$ e $j=1, R(z)=\frac{1+\frac{z}{2}}{1-\frac{z}{2}}$, que está relacionada à regra do trapézio $y_{n+1}-y_{n}=\frac{h}{2}\left(f_{n+1}+f_{n}\right)$;

2. para $k=0$ e $j=1, R(z)=\frac{1}{1-z}$, que está relacionada ao método de Euler implícito $y_{n+1}-y_{n}=h f_{n+1}$.

O E-polinômio que foi citado anteriormente é definido por

$$
E(y)=\left|Q_{j}(i y)\right|^{2}-\left|P_{k}(i y)\right|^{2}
$$

Supondo que $P_{k}(z)$ e $Q_{j}(z)$ não tenham zeros em comum, a região de estabilidade de $R$ é definida pelo conjunto

$$
D=\{z \in \mathbb{C}|| R(z) \mid \leq 1\}
$$

Definição 3.3. A aproximação $R$ é A-aceitável (A-acceptable) e então o correspondente método é A-estável se

$$
D \supset \mathbb{C}^{-}=\{z \in \mathbb{C} \mid \operatorname{Re}(z) \leq 0\}
$$

Sejam as regiões

$$
\begin{aligned}
& A_{+}=\left\{z \in \mathbb{C}|| R(z)|>| e^{z} \mid\right\}=\{z \in \mathbb{C}|| S(z) \mid>1\} \mathrm{e} \\
& A_{-}=\left\{z \in \mathbb{C}|| R(z)|<| e^{z} \mid\right\}=\{z \in \mathbb{C}|| S(z) \mid<1\}
\end{aligned}
$$

onde $S(z)=\frac{R(z)}{e^{z}}$ 
Observe que $R(z)$ e $S(z)$ têm os mesmos zeros e pólos.

Proposição 3.1. A aproximação $R$ é A-aceitável (A-acceptable) se, e somente se,

1. $A_{+}$não tem intersecção com o eixo imaginário;

2. R não tem pólos em $\mathbb{C}^{-}$.

A demonstração dessa proposição encontra-se em Wanner et al. (1978).

Definição 3.4. A aproximação $R$ tem ordem $p$ se existe uma constante $C \neq 0$ tal que

$$
e^{z}-R(z)=C z^{p+1}+O\left(z^{p+2}\right) \text { para } z \rightarrow 0 .
$$

A próxima proposição relaciona os formatos de $A_{+}$e $A_{-}$com a ordem da aproximação $R$. A demonstração encontra-se em Wanner et al. (1978).

Proposição 3.2. R é uma aproximação de ordem p se, e somente se, para $z \rightarrow 0, A_{+}$consiste de $p+1$ setores de largura $\frac{\pi}{p+1}$, separados por $p+1$ setores de $A_{-}$, cada um com mesma largura.

Os conjuntos $A_{+}$e $A_{-}$recebem o nome, respectivamente, de order star e order star dual.

A nomenclatura order star surgiu devido a aparência com uma estrela com $p+1$ setores que é produzida pelo termo

$$
e^{z}-R(z)=C z^{p+1}+O\left(z^{p+2}\right) \text { ou } 1-\frac{R(z)}{e^{z}}=C z^{p+1}+O\left(z^{p+2}\right)
$$

que determina a ordem $p$ da aproximação $R$.

Proposição 3.3. O número de zeros (pólos) na região limitada pelas componentes de $A_{+}\left(A_{-}\right)$é igual ao número de pontos de interpolação, isto é, pontos tais que $R(z)=e^{z}$, sobre a fronteira orientada, contando suas multiplicidades.

A demonstração desta proposição encontra-se em Wanner et al. (1978).

As regiões limitadas pelas componentes de $A_{+}$e $A_{-}$recebem o nome, respectivamente, de dedos (fingers) e dedos duais (dual fingers) da order star.

Nas figuras, a região $A_{+}$é representada pela área escura e a região $A_{-}$pela área em branco. $\mathrm{A}$ legenda a seguir representa os elementos das order stars.

$\diamond:$ Pólos da aproximação;

$\square$ : Zeros da aproximação;

$\times$ : Pólos da função;

+ : Zeros da função;

○: Pontos de interpolação.

A Figura 3.1 representa as order stars da aproximação de Padé para alguns valores de $j$ e $k$. 

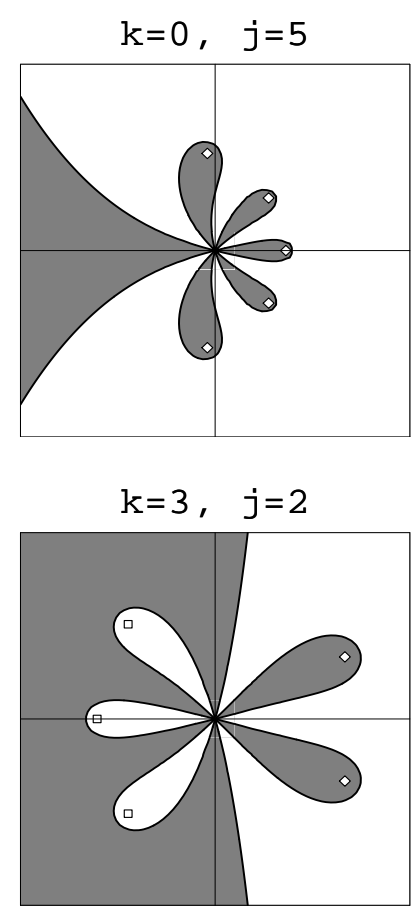

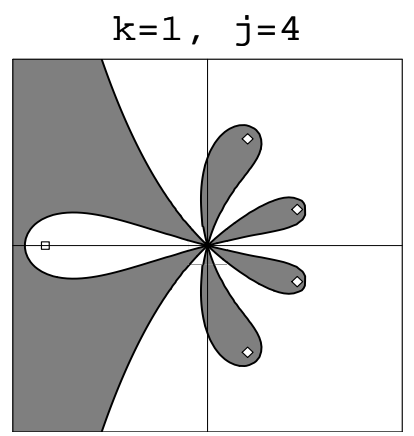

$$
\mathrm{k}=4, \mathrm{j}=1
$$

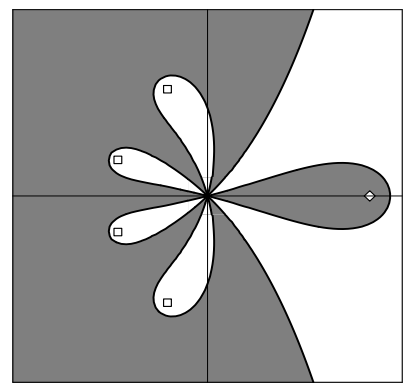

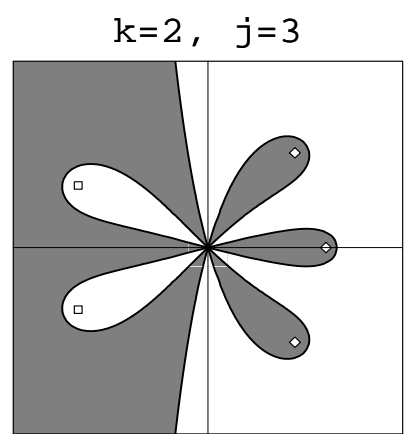

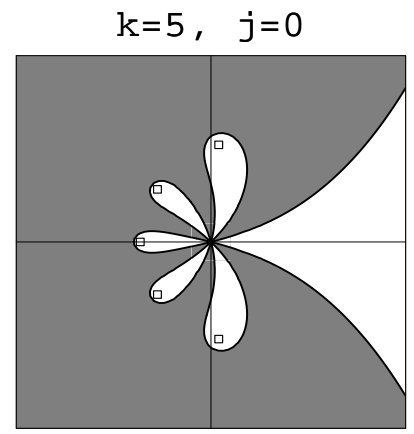

Figura 3.1: Order stars da aproximação de Padé

\subsubsection{Conceito geral}

São apresentadas agora definições e conceitos gerais sobre as order stars, que encontram-se em Iserles e Norsett (1991).

Seja $\prod_{m / n}$ o conjunto de todas as funções racionais da forma $\frac{P}{Q}$, onde $\operatorname{grau}(P) \leq m$, $\operatorname{grau}(Q) \leq n$ e $Q(0)=1$

Uma função complexa é chamada de essencialmente analítica se é meromorfa no plano complexo fechado $\operatorname{cl} \mathbb{C}:=\mathbb{C} \cup\{\infty\}$, exceto talvez num conjunto finito de singularidades essenciais.

Seja $f$ uma função essencialmente analítica que pode ser aproximada pela função racional $R \in \prod_{m / n}$. Excluindo o caso em que $R \equiv f$, seja

$$
\mu(z):=\frac{R(z)}{f(z)}, z \in \mathrm{cl} \mathbb{C}
$$

onde $\mu$ é essencialmente analítica.

A order star de primeira espécie de $\{f, R\}$ é definida como sendo a partição $\left\{A_{+}, A_{0}, A_{-}\right\}$do plano complexo fechado, onde

$$
\begin{aligned}
& A_{+}:=\{z \in c l \mathbb{C}|| \mu(z) \mid>1\}, \\
& A_{0}:=\{z \in c l \mathbb{C}|| \mu(z) \mid=1\}, \\
& A_{-}:=\{z \in c l \mathbb{C}|| \mu(z) \mid<1\} .
\end{aligned}
$$


Definição 3.5. O ponto $z_{0} \in c l \mathbb{C}$ é chamado de ponto de interpolação de grau $p \geq 1$ se $f$ é analítica numa vizinhança de $z_{0} e$

$$
\begin{array}{lll}
\left|z_{0}\right|<\infty & : \quad R(z)=f(z)+C\left(z-z_{0}\right)^{p}+O\left(\left|z-z_{0}\right|^{p+1}\right) \\
\left|z_{0}\right|=\infty & : \quad R(z)=f(z)+C z^{-p}+O\left(|z|^{-p-1}\right),
\end{array}
$$

onde $C \neq 0$ e $z_{0} \in A_{0}$.

O grau de interpolação pode ser analisado pela geometria das order stars através do número de setores de $A_{+}$e $A_{-}$que aproximam-se de $z_{0}$.

Definição 3.6. $O$ índice $\iota(z)$ de um ponto $z \in A_{0}$ é definido como sendo o número de setores de A- adjacente a $z$.

Sejam $z \in A_{0}$ e $\wp(z)$ o conjunto de todos os inteiros não-negativos $k$ que possuem a propriedade de que, para todo $\epsilon>0$, existem $\delta \in(0, \epsilon]$ e exatamente $k$ arcos do círculo $\{\zeta \in \mathbb{C}|| \zeta-z \mid=\delta\}$ que pertencem a $A_{-}$. Além disso, se $\infty \in A_{0}$, então $\wp(\infty)$ é o conjunto de todos os números inteiros não-negativos que possuem a propriedade de que para todo $r>0$, existem $s \geq r$ e exatamente $k$ arcos do círculo $\{z \in \mathbb{C}|| z \mid=s\}$ que pertencem a $A_{-}$. Assim, o índice de $z \in A_{0}$ é definido por

$$
\iota(z):=\min \{k \mid k \in \wp(z)\}
$$

Sejam $z \in A_{0}$ e $p=\iota(z)>0$. Se $\mu$ é analítica em $z$ e o ponto é aproximado por precisamente $p$ setores de $A_{-}$e $p$ setores de $A_{+}$com cada ângulo assintótico de $\frac{\pi}{p}$, segue que $z$ é regular.

Proposição 3.4. Seja $z_{0}$ um zero de $f$ de multiplicidade $k \leq 0$ (inclusive o caso $k=0$, ou seja, $\left.f\left(z_{0}\right) \neq 0\right)$. Se $z_{0}$ é um ponto de interpolação de grau $p \geq \max \{1, k+1\}$ então $\iota\left(z_{0}\right)=p-k$ e $z_{0}$ é regular.

A demonstração desse resultado encontra-se em Iserles e Norsett (1991).

Seja $V$ um subconjunto aberto de clC tal que $f$ é analítica em $V$ e $|f| \equiv 1$ ao longo da fronteira de $V$, exceto talvez por um número finito de sigularidades essenciais. Pelo princípio do módulo máximo, uma função analítica atinge seu valor máximo na fronteira. Logo, $|f(z)|<1$ para todo $z \in V$ e $f$ mapeia $\mathrm{cl} V$ no disco unitário. Tal função recebe o nome de $V^{*}$-contração. Além disso, qualquer função que analiticamente mapeia $V$ no interior do disco unitário é chamada de $V$-contração.

Proposição 3.5. Seja $f$ uma $V^{*}$-contração. Então $R$ é uma $V$-contração se, e somente se, é analítica em $V$ e $A_{+} \cap \partial V=\emptyset$. 
Demonstração: Como $f$ é analítica em $V$, então $\mu$ é analítica em qualquer domínio fechado de $V$. Na fronteira de $V,|\mu(z)|=|R(z)|$, exceto possivelmente num conjunto finito de pontos. Então, pelo do princípio do máximo, segue o resultado.

Os componentes de $A_{+}$e de $A_{-}$são chamados de $A_{+}$-regiões e $A_{-}$-regiões, respectivamente. Tal região é dita analítica se $\mu$ é analítica ao longo de sua fronteira.

Observe que os pontos de interpolação encontram-se em $A_{0}$, que é o encontro das fronteiras de $A_{+}$e $A_{-}$. A região $A_{+}$ou $A_{-}$tem multiplicidade $L$ se sua fronteira direcionada passa por exatamente $L$ pontos de interpolação, que não precisam ser distintos.

Proposição 3.6. A multiplicidade $L$ da região analítica $A_{+}$é igual ao número de pólos de $\mu$, contando suas multiplicidades, dentro do domínio, $e 1 \leq L<\infty$. A multiplicidade $L$ da região analítica $A_{-}$é igual ao número de zeros de $\mu$, contando suas multiplicidades, dentro do domínio, $e 1 \leq L<\infty$.

A demonstração dessa proposição encontra-se em Iserles e Norsett (1991).

A seguir é apresentada outra técnica para a obtenção das order stars, que recebe o nome de order stars de segunda espécie.

Sejam $f$ uma função complexa, $R$ uma aproximação de $f$ e

$$
\widetilde{\mu}(z):=R(z)-f(z), z \in \operatorname{cl} \mathbb{C},
$$

onde $f$ e $R$ estão bem definidas.

Definição 3.7. A order star de segunda espécie é a tripla $\left\{\widetilde{A}_{+}, \widetilde{A}_{0}, \widetilde{A}_{-}\right\}$, onde

$$
\begin{aligned}
\widetilde{A}_{+} & =\{z \mid \operatorname{Re}(\widetilde{\mu}(z))>0\} \\
\widetilde{A}_{0} & =\{z \mid \operatorname{Re}(\widetilde{\mu}(z))=0\} \\
\widetilde{A}_{-} & =\{z \mid \operatorname{Re}(\widetilde{\mu}(z))<0\} .
\end{aligned}
$$

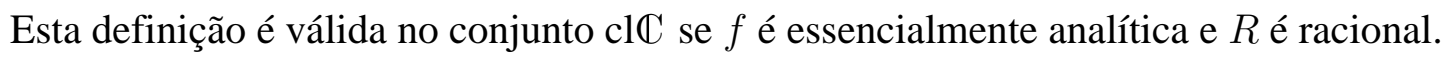

A seguir é estabelecida a relação entre order stars de primeira e segunda espécies.

Lema 3.1. Sejam $\widetilde{f}$ uma função, $\widetilde{R}$ uma aproximação de $\widetilde{f}, f(z):=e^{\widetilde{f}(z)}, R(z):=e^{\widetilde{R}(z)}$, $\mu(z):=\frac{R(z)}{f(z)}$ e $\widetilde{\mu}(z):=\widetilde{R}(z)-\widetilde{f}(z)$. A order star de primeira espécie de $\mu$ é precisamente a order star de segunda espécie de $\widetilde{\mu}$.

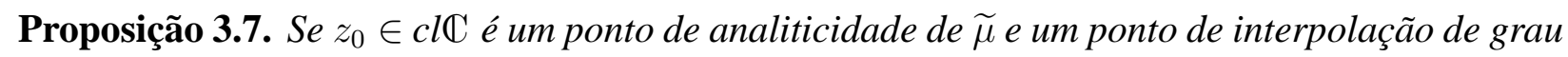
p então $z_{0} \in \widetilde{A}_{0}, \iota\left(z_{0}\right)=$ pe $z_{0}$ é regular.

A demonstração dessa proposição encontra-se em Iserles e Norsett (1991).

Uma curva fechada, positivamente orientada, de $\widetilde{A}_{0}$ é um $A_{+}$-laço se é limitada por dentro por $\widetilde{A}_{+}$, e um $A_{-}$-laço se é limitada por dentro por $\widetilde{A}_{-}$. Um laço tem multiplicidade $L$ se contém $L$ pontos de interpolação. 
Proposição 3.8. A multiplicidade de um $A_{+}$-laço ou $A_{-}$-laço é igual ao número de singularidades de $\widetilde{\mu}(z)$.

Proposição 3.9. Seja $z_{0}$ um pólo de $\widetilde{\mu}(z)$ de multiplicidade $m$. Então $\iota\left(z_{0}\right)=m$ e $z_{0}$ é regular.

No caso das singularidades, muitos resultados vistos anteriormente são irrelevantes para as order stars de segunda espécie, pois singularidades essenciais de $\widetilde{\mu}$ de ordem limitada são transformadas em singularidades essenciais de $\mu$ de ordem infinita. Então, o comportamento próximo às singularidades essenciais deve ser estudado caso a caso.

Como será necessário o uso das order stars no estudo das propriedades dos métodos $(K, L)$ de Brown, a próxima seção traz uma abordagem desta técnica para os métodos de passo múltiplo.

\subsubsection{Order stars para os métodos de passo múltiplo}

Considere o problema de valor inicial

$$
y^{\prime}=f(x, y), x \geq x_{0}, y \in \mathbb{R}^{n}
$$

e sua solução numérica $y_{n}$ em $x_{n}=x_{0}+n h, n \geq 0, h>0$, por um método convergente.

A seguir são apresentados alguns resultados sobre order stars para o método linear de passo múltiplo, dado por

$$
\sum_{j=0}^{k} \alpha_{j} y_{n+j}=h \sum_{j=0}^{k} \beta_{j} f_{n+j},
$$

que representa uma solução numérica para o problema (3.2.1).

Sejam $\rho(z)$ e $\sigma(z)$ o primeiro e o segundo polinômios característicos relacionados ao método (3.2.2), respectivamente, ou seja:

$$
\rho(z)=\sum_{j=0}^{k} \alpha_{j} z^{j} \text { e } \sigma(z)=\sum_{j=0}^{k} \beta_{j} z^{j}
$$

O polinômio característico do método (3.2.2) é dado por

$$
\pi(w, z)=\rho(w)-z \sigma(w)
$$

Sabe-se que o método (3.2.2) tem ordem $p$ se

$$
\rho\left(e^{h}\right)-h \sigma\left(e^{h}\right)=O\left(h^{p+1}\right) .
$$


Em seu artigo, Iserles e Norsett (1984) definiram a função geradora das order stars para o método linear de passo múltiplo por

$$
\widetilde{\mu}(z)=\frac{\sigma\left(e^{z}\right)}{\rho\left(e^{z}\right)}-\frac{1}{z}, z \in \mathbb{C},
$$

sendo

$$
\begin{aligned}
\widetilde{A}_{+} & =\{z \mid \operatorname{Re}(\widetilde{\mu}(z))>0\} \\
\widetilde{A}_{0} & =\{z \mid \operatorname{Re}(\widetilde{\mu}(z))=0\} \mathrm{e} \\
\widetilde{A}_{-} & =\{z \mid \operatorname{Re}(\widetilde{\mu}(z))<0\}
\end{aligned}
$$

os conjuntos que definem a order star de segunda espécie.

Os métodos $(K, L)$ de Brown têm ordem $p$ se

$$
\rho\left(e^{h}\right)-h\left(\sum_{j=1}^{L} \sigma_{j}\left(e^{h}\right) h^{j-1}\right)=O\left(h^{p+1}\right) .
$$

Em analogia, as order stars para os métodos $(K, L)$ de Brown devem ser geradas por

$$
\widetilde{\mu}(z)=\frac{\sum_{j=1}^{L} \sigma_{j}\left(e^{z}\right) z^{j-1}}{\rho\left(e^{z}\right)}-\frac{1}{z}, z \in C .
$$

Na seqüência serão apresentados alguns resultados da subseção anterior adaptados para o caso do método multiderivada de passo múltiplo.

O primeiro resultado relaciona a ordem do método numérico com a quantidade de setores das regiões $\widetilde{A}_{+}$e $\widetilde{A}_{-}$.

Proposição 3.10. Se o método numérico tem ordem $p$, então $p-1$ setores de $\widetilde{A}_{+}$e $p-1$ setores de $\widetilde{A}_{-}$aproximam-se da origem com ângulos assintóticos de $\frac{\pi}{p-1}$ cada.

O próximo resultado estabelece a zero-estabilidade do método numérico por meio de order stars.

Proposição 3.11. O método é zero-estável se, e somente se, os pólos de $\widetilde{\mu}(z)$ estão no semi-plano esquerdo fechado e os pólos ao longo do eixo imaginário são simples.

Convém lembrar que para demonstrar esta proposição é preciso utilizar a transformação $z \rightarrow \ln z$, que mapeia o interior do disco unitário no semi-plano $\operatorname{Re}(z)<0$ e o círculo unitário no eixo imaginário.

A $A$-estabilidade do método ou, equivalentemente, a $A$-aceitabilidade da aproximação $\widetilde{\mu}$, pode ser analisada através de order stars pelo seguinte resultado de Iserles e Norsett (1991): 
Proposição 3.12. A aproximação $\widetilde{\mu}$ é A-aceitável (A-acceptable) se, e somente se, $\widetilde{A}_{-}$não tem intersecção com o eixo imaginário.

Como a função $\tilde{\mu}(z)$ envolve $e^{z}$, que é periódica no plano complexo, os pólos e zeros são reproduzidos por múltiplos de $2 \pi i$, o que dificulta o estudo das order stars. Portanto, convém restringir a atenção à região

$$
J=\{z \in \mathbb{C}:|\operatorname{Im}(z)| \leq \pi\}
$$

Sejam os conjuntos

$$
\begin{aligned}
& J^{+}=\{z \in J: \operatorname{Re}(z)>0\} \mathrm{e} \\
& J^{-}=\{z \in J: \operatorname{Re}(z)<0\} .
\end{aligned}
$$

O próximo resultado determina a posição relativa entre os zeros e os pólos de $\widetilde{\mu}(z)$.

Proposição 3.13. Seja $\gamma$ um laço tal que $\gamma \cap \partial J=\emptyset$ e $\gamma \cap J \neq \emptyset$. Então existe sobre $\gamma$ exatamente um pólo de $\mu$ entre duas raízes de $\mu(z)=0$. Além disso, se $z_{0} \in$ int $J$ é um pólo de $\mu$ de multiplicidade $m$, então é aproximado por $m$ setores de $\widetilde{A}_{+}$e $m$ setores de $\widetilde{A}_{-}$com ângulos de $\frac{\pi}{m}$ cada.

Proposição 3.14. Seja $F$ a região limitada por $\widetilde{A}_{+}$ou a região limitada por $\widetilde{A}_{-}$tal que $\{\mathbb{R}+i \pi\} \cap c l F \neq \emptyset$ e sejam

$$
\begin{aligned}
& x_{-}=\min \{x \in \mathbb{R}: x+i \pi \in c l F\}>-\infty \\
& x_{+}=\max \{x \in \mathbb{R}: x+i \pi \in c l F\}<\infty .
\end{aligned}
$$

Seja $z_{0} \in \partial F \cap$ int $J$ um zero de $\widetilde{\mu}(z)$. Então

1. se F é uma $\widetilde{A}_{-}$-região então ou $x_{-}+i \pi$ é um pólo de $\widetilde{\mu}$ ou existe um pólo de $\widetilde{\mu}$ ao longo da porção orientada positivamente de $\partial F$ de $x_{-}+i \pi$ à $z_{0}$;

2. se F é uma $\widetilde{A}_{+}$-região então ou $x_{+}+i \pi$ é um pólo de $\widetilde{\mu}$ ou existe um pólo de $\widetilde{\mu}$ ao longo da porção orientada positivamente de $\partial F$ de $x_{+}+i \pi$ à $z_{0}$.

Resultados análogos são válidos considerando $\mathbb{R}-i \pi$ no lugar de $\mathbb{R}+i \pi$.

As demonstrações dos dois resultados acima encontram-se em Iserles e Norsett (1984).

\subsection{Propriedades dos métodos de Brown e order stars}

São apresentadas, primeiramente, as order stars para os métodos $(K, L)$ de Brown para alguns valores fixos de $K$ e $L$. Um estudo das order stars para os métodos BDF é apresentado e depois a 
equação (3.2.4), que generaliza o conceito de order stars para os métodos $(K, L)$, será usada para obter as order stars para $L=2$. Será possível verificar muitas propriedades desses métodos, como, por exemplo, $A$-estabilidade e zero-estabilidade, através das order stars.

\subsubsection{Order stars para os métodos $(K, L)$}

É apresentada, a seguir, uma análise das propriedades dos métodos BDF utilizando order stars.

Para analisar a convergência dos métodos BDF, convém determinar a consistência e a zeroestabilidade. Como esses métodos têm ordem de consistência $p=K$, é apresentada aqui somente a análise da zero-estabilidade, pois tais métodos são sempre consistentes.

No caso dos métodos BDF, a função geradora das order stars é dada por

$$
\widetilde{\mu}(z)=\frac{\sigma\left(e^{z}\right)}{\rho\left(e^{z}\right)}-\frac{1}{z} .
$$

Portanto:

1. $K=2: \widetilde{\mu}(z)=\frac{\left(\frac{2}{3} z-1\right) e^{2 z}+\frac{4}{3} e^{z}-\frac{1}{3}}{z\left(e^{2 z}-\frac{4}{3} e^{z}+\frac{1}{3}\right)}$;

2. $K=3: \widetilde{\mu}(z)=\frac{\left(\frac{18}{33} z-1\right) e^{3 z}+\frac{18}{11} e^{2 z}-\frac{9}{11} e^{z}+\frac{2}{11}}{z\left(e^{3 z}-\frac{18}{11} e^{2 z}+\frac{9}{11} e^{z}-\frac{2}{11}\right)}$;

3. $K=4: \widetilde{\mu}(z)=\frac{\left(\frac{12}{25} z-1\right) e^{4 z}+\frac{48}{25} e^{3 z}-\frac{36}{25} e^{2 z}+\frac{16}{25} e^{z}-\frac{3}{25}}{z\left(e^{4 z}-\frac{48}{25} e^{3 z}+\frac{36}{25} e^{2 z}-\frac{16}{25} e^{z}+\frac{3}{25}\right)}$;

4. $K=5: \widetilde{\mu}(z)=\frac{\left(\frac{60}{137} z-1\right) e^{5 z}+\frac{300}{137} e^{4 z}-\frac{300}{137} e^{3 z}+\frac{200}{137} e^{2 z}-\frac{75}{137} e^{z}+\frac{12}{137}}{z\left(e^{5 z}-\frac{300}{137} e^{4 z}+\frac{300}{137} e^{3 z}-\frac{200}{137} e^{2 z}+\frac{75}{137} e^{z}-\frac{12}{137}\right)}$.

As figuras a seguir apresentam as order stars dos métodos BDF para $K=2,3,4,5,6,7,8,9$ e 10 , respectivamente.

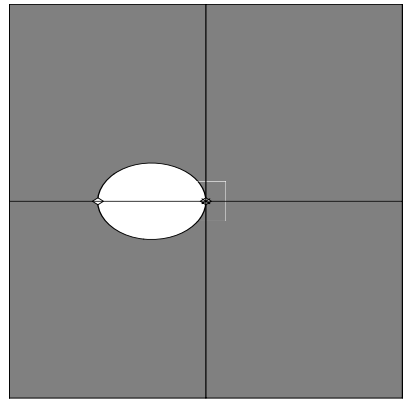

Figura 3.2: Order star do BDF de 2 passos

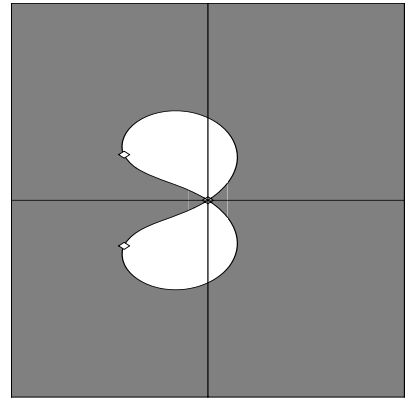

Figura 3.3: Order star do BDF de 3 passos

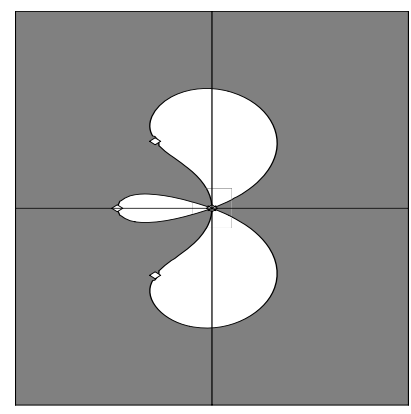

Figura 3.4: Order star do BDF de 4 passos 


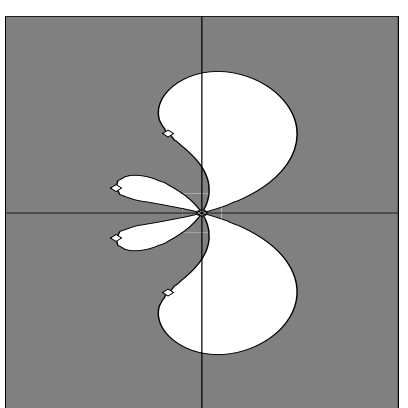

Figura 3.5: Order star do BDF de 5 passos

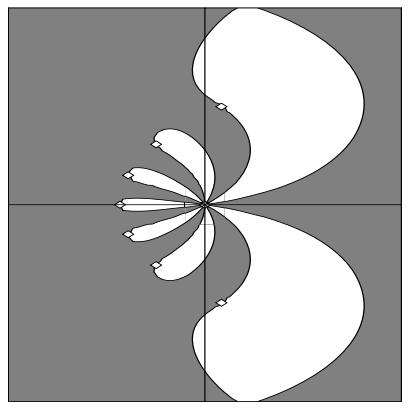

Figura 3.8: Order star do BDF de 8 passos

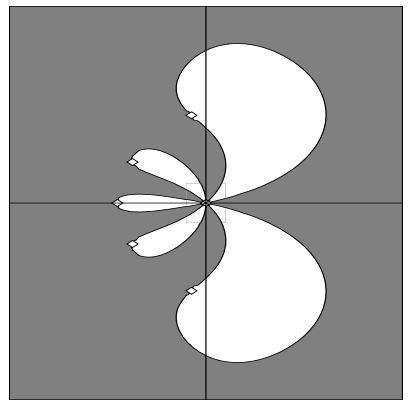

Figura 3.6: Order star do BDF de 6 passos

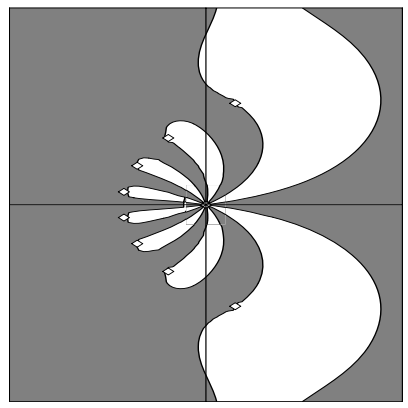

Figura 3.9: Order star do BDF de 9 passos

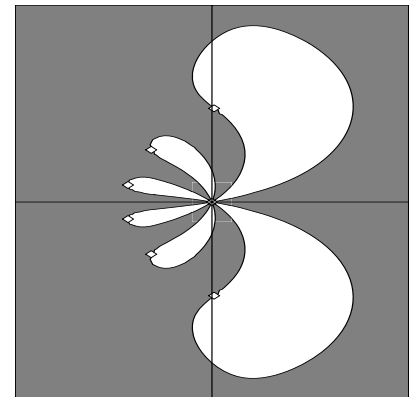

Figura 3.7: Order star do BDF de 7 passos

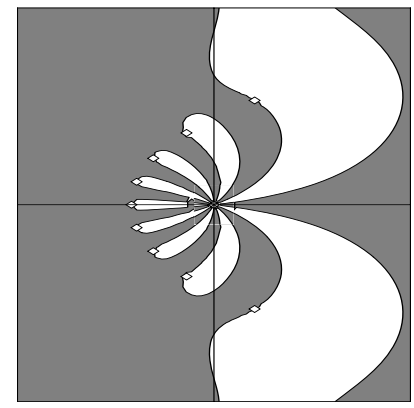

Figura 3.10: Order star do BDF de 10 passos

É possível observar que cada order star possui $p-1=K-1$ setores, onde $p=K$ é a ordem do método. Para $K=2, \widetilde{A}_{-} \cap\{i \mathbb{R}\}=\emptyset$ e para $K \geq 3, \widetilde{A}_{-} \cap\{i \mathbb{R}\} \neq \emptyset$. Portanto, os métodos BDF são $A$-estáveis somente quando $K \leq 2$, o que já foi estabelecido pelo Teorema 3.2.

O ponto $z_{0}=0$ é um ponto de interpolação de grau $p=K-1$, pois $K-1$ setores de $\widetilde{A}_{-}$ aproximam-se de $z_{0}=0$. Além disso, $\iota(0)=K-1$. Então, pela Proposição 3.9, segue que $z_{0}=0$ é regular.

A partir de $K=7$, existem pólos da aproximação $\widetilde{\mu}(z)$ que encontram-se no semi-plano direito. Logo, os métodos BDF são zero-estáveis apenas para $K \leq 6$. Portanto, como os métodos BDF são consistentes, segue que são convergentes somente para $K \leq 6$.

De (3.2.4) sabe-se que:

$$
\widetilde{\mu}(z)=\frac{\sum_{j=1}^{L} \sigma_{j}\left(e^{z}\right) z^{j-1}}{\rho\left(e^{z}\right)}-\frac{1}{z}, z \in C .
$$

Para facilitar a compreensão das order stars para os métodos $(K, L)$ no caso geral, será feita a seguir a análise para a classe dos métodos $(K, 2)$. Os polinômios característicos dos métodos 
$(K, 2)$ são dados por

$$
\rho(\xi)=\sum_{i=0}^{K} \alpha_{i} \xi^{i}, \sigma_{1}(\xi)=\beta_{1} \xi^{K} \text { e } \sigma_{2}(\xi)=\beta_{2} \xi^{K}
$$

As order stars para os métodos $(K, 2)$ são, portanto, obtidas de:

$$
\widetilde{\mu}(z)=\frac{\sigma_{1}\left(e^{z}\right)+z \sigma_{2}\left(e^{z}\right)}{\rho\left(e^{z}\right)}-\frac{1}{z}, z \in C
$$

As Figuras 3.11, 3.12, 3.13, 3.14, 3.15 e 3.16 apresentam as order stars dos métodos $(K, 2)$ de Brown para $K=3,4,5,10,11$ e 12 , respectivamente.

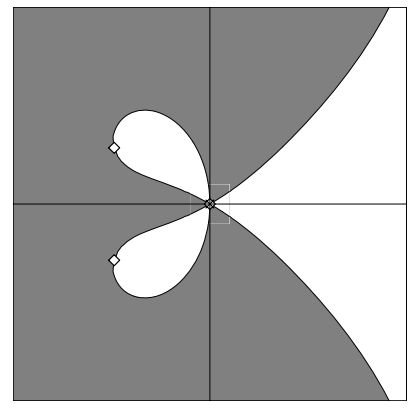

Figura 3.11: Order star do método $(3,2)$ de Brown

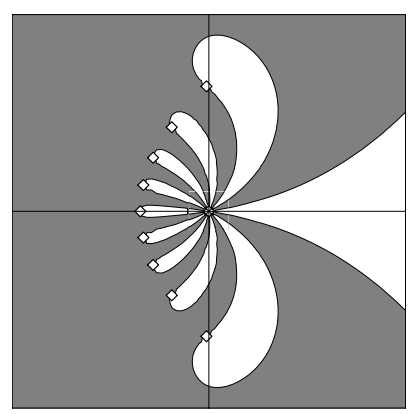

Figura 3.14: Order star do método $(10,2)$ de Brown

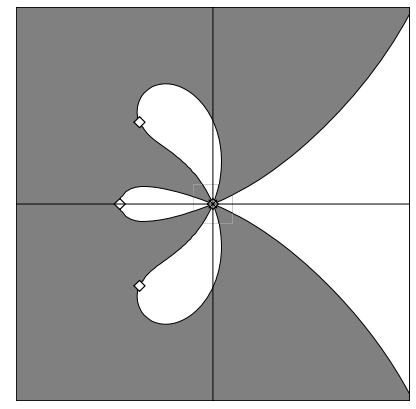

Figura 3.12: Order star do método $(4,2)$ de Brown

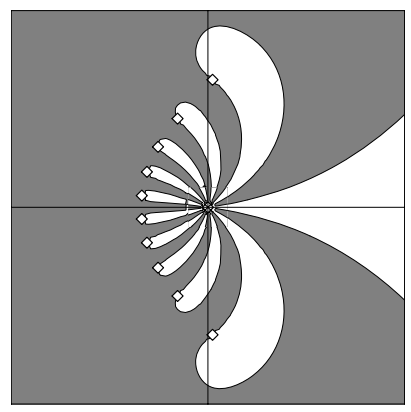

Figura 3.15: Order star do método $(11,2)$ de Brown

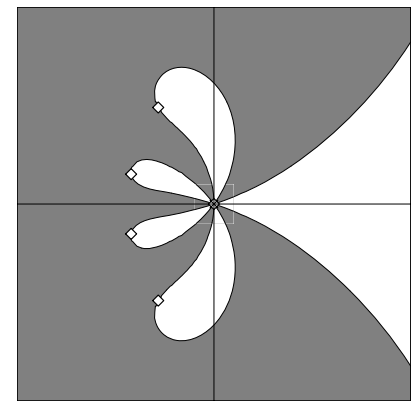

Figura 3.13: Order star do método $(5,2)$ de Brown

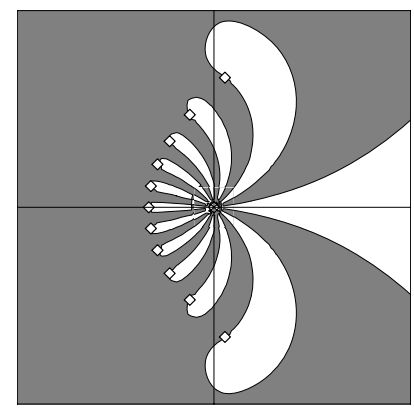

Figura 3.16: Order star do método $(12,2)$ de Brown

Para o caso do método $(4,2)$ de Brown, dado por

$$
y_{n+4}-\frac{576}{415} y_{n+3}+\frac{216}{415} y_{n+2}-\frac{64}{415} y_{n+1}+\frac{9}{415} y_{n}=\frac{300}{415} h f_{n+5}-\frac{72}{415} h^{2} f_{n+5}^{\prime}
$$

cuja ordem é $p=K+1=5$, a order star tem quatro setores. A aproximação $\widetilde{\mu}$ para esse método é:

$$
\widetilde{\mu}(z)=\frac{\left(-\frac{72}{415} z^{2}+\frac{60}{83} z-1\right) e^{4 z}+\frac{576}{415} e^{3 z}-\frac{216}{415} e^{2 z}+\frac{64}{415} e^{z}-\frac{9}{415}}{z\left(e^{4 z}-\frac{576}{415} e^{3 z}+\frac{216}{415} e^{2 z}-\frac{64}{415} e^{z}+\frac{9}{415}\right)} .
$$


Além disso, é possível observar das figuras anteriores que para $K=3, A_{-} \cap\{i \mathbb{R}\}=\emptyset$ e para $K \geq 4, A_{-} \cap\{i \mathbb{R}\} \neq \emptyset$. Assim, pela Proposição 3.12, os métodos $(K, 2)$ de Brown são $A$-estáveis somente quando $p \leq 4$, isto é, $K \leq 3$, o que já se sabia tendo em vista o Teorema 3.2.

O ponto $z_{0}=0$ é um ponto de interpolação de grau $p=K$, pois $K$ setores de $\widetilde{A}_{-}$aproximamse de $z_{0}=0$. Além disso, $\iota(0)=K$ e $z_{0}=0$ é regular.

A partir de $K=11$ pode-se observar que existem pólos da aproximação $\widetilde{\mu}$ no semi-plano direito. Portanto os métodos $(K, 2)$ de Brown são zero-estáveis para $K \leq 10$ apenas. Como tais métodos são consistentes, segue, pelo clássico resultado de Dahlquist, que os métodos $(K, 2)$ de Brown são convergentes somente para $K \leq 10$.

No caso geral, as order stars para os métodos $(K, L)$ de Brown possuem $K+L-2$ setores que aproximam-se da origem com ângulos assintóticos de $\frac{\pi}{K+L-2}$, conforme a Proposição 3.10, pois tais métodos têm ordem $p=K+L-1$.

Observe que

$$
\begin{aligned}
\widetilde{\mu}\left(\frac{1}{\xi}\right) & =\frac{\sigma\left(e^{1 / \xi}\right)}{\rho\left(e^{1 / \xi}\right)}-\xi=\frac{\sigma\left(e^{1 / \xi}\right)-\xi \rho\left(e^{1 / \xi}\right)}{\rho\left(e^{1 / \xi}\right)} \\
& =\frac{e^{K / \xi}\left(\beta_{1}+\beta_{2}\left(\frac{1}{\xi}\right)+\ldots+\beta_{L}\left(\frac{1}{\xi}\right)^{L-1}\right)-\xi\left(\alpha_{0}+\alpha_{1} e^{1 / \xi}+\ldots+\alpha_{K} e^{K / \xi}\right)}{\alpha_{0}+\alpha_{1} e^{1 / \xi}+\ldots+\alpha_{K} e^{K / \xi}} \\
& =\frac{\beta_{1}+\beta_{2}\left(\frac{1}{\xi}\right)+\ldots+\beta_{L}\left(\frac{1}{\xi}\right)^{L-1}-\xi\left(\frac{\alpha_{0}}{e^{K / \xi}}+\ldots+\alpha_{K}\right)}{\frac{\alpha_{0}}{e^{K / \xi}}+\ldots+\alpha_{K}} .
\end{aligned}
$$

Logo,

$$
\lim _{\xi \rightarrow 0} \xi^{L-1} \widetilde{\mu}\left(\frac{1}{\xi}\right)=\frac{\beta_{L}}{\alpha_{K}}
$$

o que caracteriza que 0 é um pólo de ordem $L-1$ de $\widetilde{\mu}\left(\frac{1}{\xi}\right)$ e então $z_{0}=\infty$ é um pólo de ordem $L-1$ de $\widetilde{\mu}(z)$.

Portanto, da Proposição 3.9 segue que $\iota(\infty)=L-1$.

Observe que

$$
\iota(0)=K+L-2=(K-1)+(L-1) .
$$

Portanto, $(K-1)+(L-1)$ setores de $\widetilde{A}_{-}$aproximam-se da origem, sendo $L-1$ setores obtidos através de $\iota(\infty)=L-1$ e $K-1$ aqueles onde encontram-se os pólos da aproximação $\widetilde{\mu}(z)$.

Um estudo detalhado sobre tal comportamento das order stars para os métodos de Brown pode ser visto na próxima subseção. 


\subsubsection{Comportamento dos zeros dos polinômios característicos}

A seguir é mostrado que os zeros dos polinômios característicos associados aos métodos $(K, L)$ de Brown são distintos. Primeiramente é considerado o caso em que $L=1$, que são os métodos $\mathrm{BDF}$, e depois a validade deste resultado no caso geral é apresentado.

Proposição 3.15. Os zeros dos polinômios característicos associados aos métodos BDF são distintos.

Demonstração: Observe primeiramente que $z_{0}=\infty$ é um pólo de ordem 0 de $\widetilde{\mu}(z)$, pois $\xi=0$ é um pólo de ordem 0 de $\widetilde{\mu}\left(\frac{1}{\xi}\right)$. Portanto, da Proposição 3.9 segue que $\iota(\infty)=0$, como já foi citado anteriormente.

Logo, as order stars para os métodos BDF possuem $K-1$ setores de $\widetilde{A}_{-}$que aproximam-se da origem, sendo $\iota(\infty)=0$, o que significa que a aproximação não possui setores onde encontram-se os zeros de $\widetilde{\mu}(z)$. Portanto, as order stars para os métodos BDF possuem $K-1$ laços onde estão os pólos de $\widetilde{\mu}(z)$. Além disso, $z_{0}=0$ é uma raiz de $\widetilde{\mu}(z)=0$.

É preciso analisar dois casos:

1. $\gamma \cap \partial J=\emptyset$ e $\gamma \cap J \neq \emptyset$ :

Da Proposição 3.13 segue que existe exatamente um pólo de $\widetilde{\mu}(z)$ entre duas raízes de $\widetilde{\mu}(z)=0$. Como $z_{0}=0$ é raiz de $\widetilde{\mu}(z)=0$ então cada laço possui um pólo de $\widetilde{\mu}(z)$.

As Figuras 3.2, 3.3, 3.4, 3.5, 3.6 e 3.7 ilustram este caso.

2. $\{\mathbb{R}+i \pi\} \cap \operatorname{cl} F \neq \emptyset$, onde $F$ pode ser uma $A_{+}$-região ou $A_{-}$-região:

Da Proposição 3.14 segue que existe um pólo de $\widetilde{\mu}$ sobre o laço $\gamma$ tal que $\gamma \cap \partial J \neq \emptyset$, conforme ilustrado nas Figuras 3.8, 3.9 e 3.10.

Portanto, em ambos os casos, desde que a origem é uma raiz de $\widetilde{\mu}(z)=0$, cada laço possui um pólo de $\widetilde{\mu}(z)$, ou seja, uma raiz de $\rho(z)=0$.

Como já foi observado anteriormente, as order stars dos métodos BDF possuem $K-1$ laços. Como cada setor possui uma raiz de $\rho(z)=0$, é possível concluir que $\rho(z)=0$ possui $K$ raízes distintas, já que $K-1$ raízes encontram-se nos laços das order stars e a outra raiz é a raiz principal $z_{0}=1$ (representada na origem de cada figura). Portanto, $\rho(z)=0$ possui $K$ raízes distintas.

Como generalização do resultado acima, pode-se enunciar:

Proposição 3.16. Os zeros dos polinômios característicos associados aos métodos $(K, L)$ de Brown são distintos.

Demonstração: Como já foi observado anteriormente, $z_{0}=\infty$ é um pólo de ordem $L-1$ de $\widetilde{\mu}(z)$. Portanto, da Proposição 3.9 segue que $\iota(\infty)=L-1$. Como $\iota(0)=(K-1)+(L-1)$ e 
$\iota(\infty)=L-1$, então os pólos de $\widetilde{\mu}(\xi)$ encontram-se em $K-1$ setores de $\widetilde{A}_{-}$. Este fato pode ser observado nas Figuras 3.17 e 3.18.

Portanto, existe exatamente um pólo de $\widetilde{\mu}$ em cada um dos $K-1$ laços da order star e o outro zero é $z_{0}=1$, que é a raiz principal de $\rho(z)=0$. Logo, $\rho(z)$ possui $K$ zeros distintos.

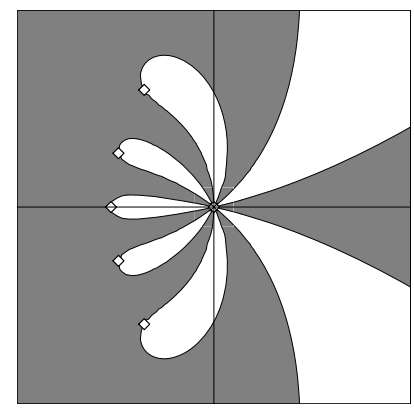

Figura 3.17: Order star do método $(6,3)$ de Brown

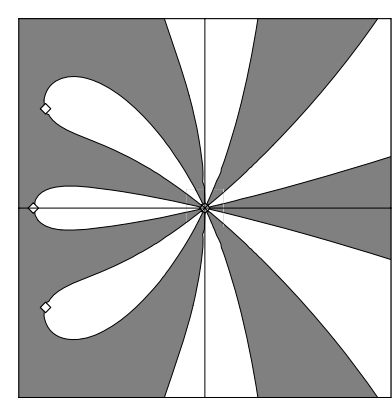

Figura 3.18: Order star do método $(4,5)$ de Brown

É fácil verificar numericamente que os zeros dos polinômios característicos associados aos métodos $(K, L)$ de Brown são distintos. Mas uma demonstração deste fato parece não existir na literatura. Portanto, este resultado é uma contribuição desta tese de interesse teórico que vem esclarecer esse ponto.

Além disso, o uso das order stars para demonstrar resultados deste tipo é ainda uma técnica pouco utilizada. Portanto, este resultado poderá ser útil na análise de questões relacionadas a outros métodos numéricos.

\subsubsection{Resultados sobre zero-estabilidade}

A seguir é analisada, primeiramente, uma propriedade dos polinômios característicos associados aos métodos $(K, L)$ de Brown, que é a seguinte:

$$
\rho_{K+1}^{\prime}=(K+1)\left(\rho_{K}+\frac{1}{K+1} z^{K}\right)
$$

onde $\rho_{K}(z)$ e $\rho_{K+1}(z)$ representam os polinômios característicos dos métodos $(K, L)$ e $(K+1, L)$, respectivamente.

Este resultado permite determinar métodos instáveis, pois se para um determinado $\bar{K}$ o método é instável, então este será instável para qualquer $K \geq \bar{K}$. De fato: como $\rho_{K+1}^{\prime} \simeq \rho_{K}$ e lembrando que qualquer disco que contém os zeros de um polinômio contém também os zeros da derivada, então, supondo que $\rho_{K}(z)$ tenha zeros fora do disco unitário, o objetivo é mostrar que $\rho_{K}+\frac{1}{K+1} z^{K}$ também tem zeros fora do disco.

Embora o comportamento dos zeros de $\rho_{K}+\frac{1}{K+1} z^{K}$ não possa ser determinado utilizando resultados sobre zeros de polinômios, é possível, utilizando order stars, mostrar, como será feito 
logo adiante, que se um método $(\bar{K}, L)$ é instável, então qualquer método $(K, L)$, com $K \geq \bar{K}$, também será instável devido ao aumento da quantidade de setores das order stars no semi-plano esquerdo, determinando que alguns zeros de $\rho_{K}^{\prime}(z)$ encontram-se no semi-plano direito.

As figuras a seguir mostram este comportamento dos zeros de $\rho_{K+1}^{\prime}(z)$ (representado pelos pontos vermelhos) e $\rho_{K}(z)$ (representado pelos pontos pretos).

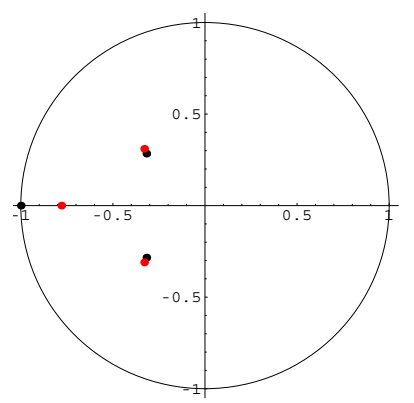

Figura 3.19: Zeros de $\rho(z)$ para $K=3 \mathrm{e}$ $\rho^{\prime}(z)$ para $K=4$, $L=1$

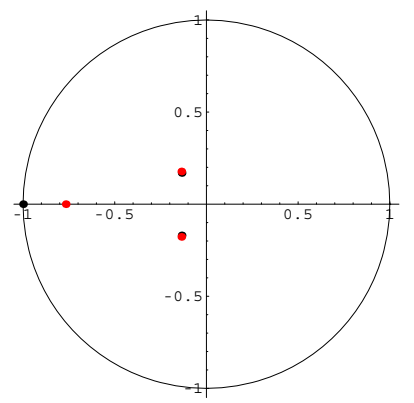

Figura 3.20: Zeros de $\rho(z)$ para $K=3 \mathrm{e}$ $\rho^{\prime}(z)$ para $K=4$, $L=2$

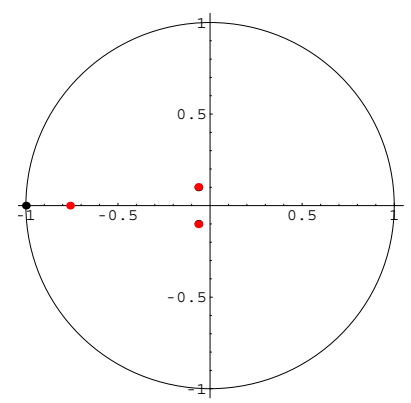

Figura 3.21: Zeros de $\rho(z)$ para $K=3 \mathrm{e}$ $\rho^{\prime}(z)$ para $K=4$, $L=3$

É possível observar, nas Figuras 3.19, 3.21 e 3.20, que os zeros de $\rho_{K}(z)$ e $\rho_{K+1}^{\prime}(z)$ estão muito próximos. É claro que, à medida que $K$ aumenta, os zeros de $\rho_{K}(z)$ e $\rho_{K+1}^{\prime}(z)$ tornam-se mais próximos.

Em relação aos zeros reais, sabe-se que, à medida que $K$ aumenta, os zeros reais de $\rho_{K+1}^{\prime}(z)$ vão se aproximando da origem. É possível observar também que a perturbação dos zeros complexos é pequena. Uma análise mais profunda será objeto de pesquisas futuras.

A seguir são apresentados dois resultados sobre zero-estabilidade dos métodos $(K, L)$ de Brown, que podem ser encontrados em Meneguette (1987). Apresentamos a demonstração destes resultados utilizando order stars, o que até onde sabemos não foi publicado na literatura.

Teorema 3.7. Seja L fixo. Os métodos $(K, L)$ de Brown tornam-se instáveis para $K$ suficientemente grande.

Demonstração: Sendo

$$
\widetilde{\mu}(z)=\frac{\sum_{j=1}^{L} \sigma_{j}\left(e^{z}\right) z^{j-1}}{\rho\left(e^{z}\right)}-\frac{1}{z}
$$

a função geradora das order stars para os métodos $(K, L)$ de Brown, segue que $\iota(\infty)=L-1$.

Assim, para um método $(K, L)$,

$$
\iota(0)=(K-1)+(L-1) \text { e } \iota(\infty)=L-1,
$$


e para um método $(K+1, L)$,

$$
\iota(0)=K+(L-1) \text { e } \iota(\infty)=L-1 .
$$

Então, como o número de setores aumenta com $K$ e cada setor contém um zero distinto de $\rho(z)$, para $K$ suficientemente grande, os setores de $\widetilde{A}_{-}$ultrapassam o eixo imaginário e dessa forma pelo menos um pólo de $\widetilde{\mu}(z)$ encontra-se no semi-plano direito, o que caracteriza que o método é instável.

Para $L$ fixo, é possível observar este comportamento relacionado à zero-estabilidade dos métodos $(K, L)$ através das order stars apresentadas na subseção anterior, ou seja, para $L=1$, por exemplo, nas Figuras 3.2, 3.3, 3.4, 3.5, 3.6, 3.7, 3.8, 3.9 e 3.10.

Teorema 3.8. Seja $K$ fixo. Os métodos $(K, L)$ de Brown tornam-se zero-estáveis para $L$ suficientemente grande.

Demonstração: Supondo que para $K$ fixo e $L$ suficientemente grande os métodos $(K, L)$ são estáveis, será provado que para um método $(K, L+1)$, o mesmo ocorre.

Então, para um método $(K, L)$,

$$
\iota(0)=(K-1)+(L-1) \text { e } \iota(\infty)=L-1,
$$

e para um método $(K, L+1)$,

$$
\iota(0)=(K-1)+L \mathrm{e} \iota(\infty)=L
$$

Portanto, a quantidade de setores do lado esquerdo não se altera.

Como pode ser visto, $\iota(\infty)$ cresce com $L$ e o mesmo ocorre com os setores a partir do semi-eixo $x$ positivo para o eixo $y$. Então, se para um método $(K, L)$ existem pólos no semi-plano positivo, pelo aumento suficiente do número de setores relacionados a $\iota(\infty)$, os pólos voltam ao semi-plano esquerdo, o que caracteriza que o método é zero-estável.

Para $K$ fixo, observe este comportamento nas Figuras 3.22, 3.23 e 3.24.

Portanto, a teoria das order stars se mostra eficiente na análise de alguns resultados já conhecidos sobre os métodos $(K, L)$ de Brown, como no caso dos teoremas apresentados nesta subseção.

\subsubsection{Regiões de estabilidade}

É ilustrado, nesta seção, um procedimento para determinar regiões de estabilidade absoluta para métodos lineares de passo múltiplo usando order stars de segunda espécie. Tal procedimento também é usado no caso dos métodos $(K, L)$ de Brown.

Para um método linear de $k$ passos, existem $k$ zeros do polinômio característico $\pi(w, z)$, representado pela equação (3.2.3), que influenciam na região de estabilidade absoluta. 


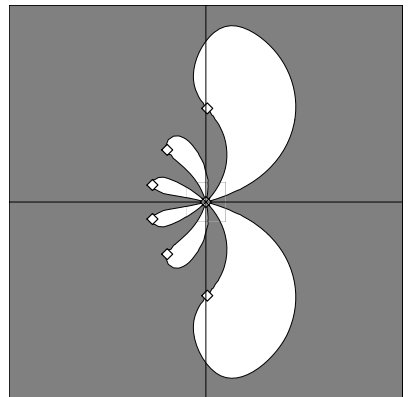

Figura 3.22: Order

star do método $(7,1)$ de Brown

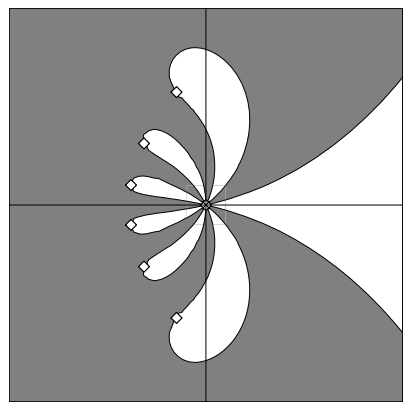

Figura 3.23: Order

star do método $(7,2)$ de Brown

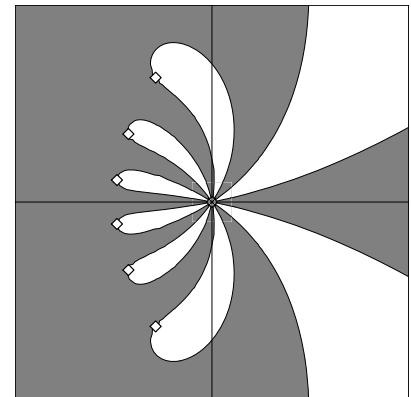

Figura 3.24: Order

star do método $(7,3)$ de Brown

Para descrever o procedimento para se obter a região de estabilidade, desenvolvido através do software Mathematica e usando o pacote OrderStar.m, cujo detalhamento encontra-se em Sofroniou (1996), considere o método BDF de 2 passos, dado por

$$
y_{n+2}-\frac{4}{3} y_{n+1}+\frac{1}{3} y_{n}=\frac{2}{3} h f_{n+2} \text {. }
$$

O objetivo é determinar as curvas soluções da equação

$$
w^{2}-\frac{4}{3} w+\frac{1}{3}-\frac{2}{3} z w^{2}=0,
$$

que neste caso são dadas por

$$
w_{+}=\frac{2+\sqrt{1+2 z}}{3-2 z}
$$

$\mathrm{e}$

$$
w_{-}=\frac{-2+\sqrt{1+2 z}}{-3+2 z}
$$

e representadas na Figura 3.25.
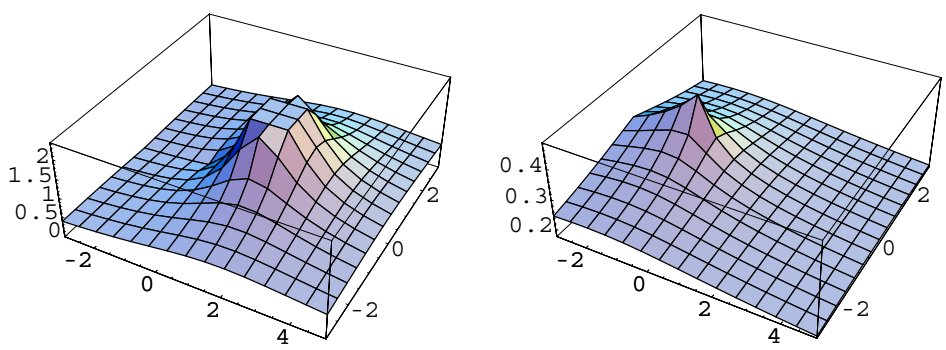

Figura 3.25: Curvas soluções para $w_{+}$e $w_{-}$para o método BDF de 2 passos

Neste caso, a notação

$$
\mathbf{w}(z)=\left[\begin{array}{l}
w_{+}(z) \\
w_{-}(z)
\end{array}\right]
$$


respresenta as curvas soluções do polinômio característico $\pi(w, z)=0$.

Para visualizar a região de estabilidade absoluta do método, é preciso combinar as duas raízes de acordo com suas magnitudes e examinar a curva de nível em $z=1$. A Figura 3.26 representa a região de estabilidade para o método $\mathrm{BDF}$ de 2 passos. A região exterior à curva é a região de estabilidade.

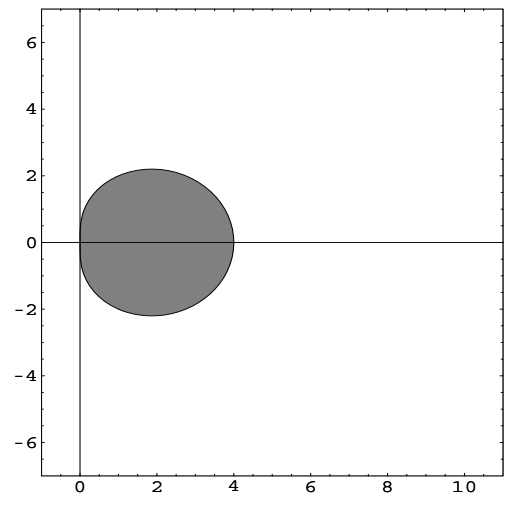

Figura 3.26: Região de estabilidade do método BDF de 2 passos

A seguir são apresentadas as regiões de estabilidade absoluta para alguns métodos $(K, L)$ para valores fixos de $K$ e $L$.

As regiões de estabilidade absoluta dos métodos BDF de 3 e 4 passos podem ser observadas nas Figuras 3.27 e 3.28, respectivamente.

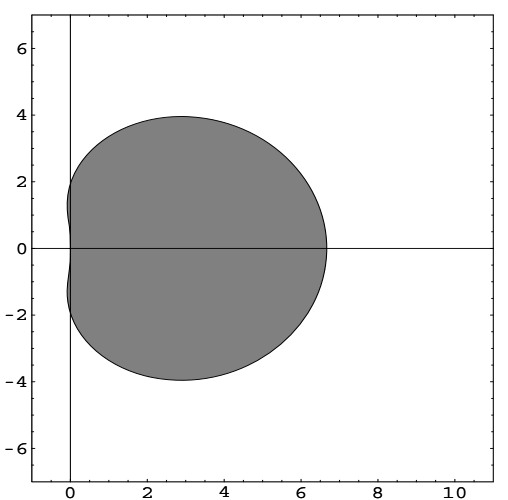

Figura 3.27: Região de estabilidade do método BDF de 3 passos

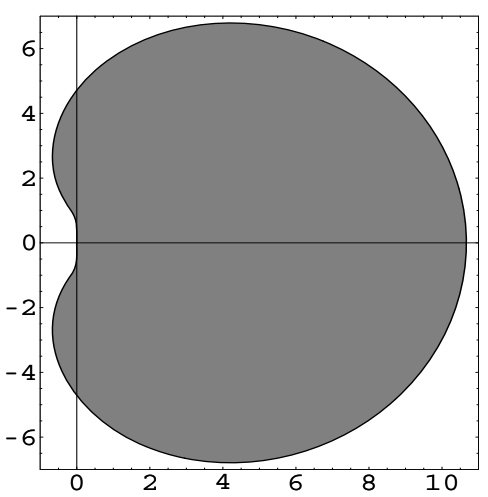

Figura 3.28: Região de estabilidade do método BDF de 4 passos

Como os métodos BDF são mais conhecidos e utilizados, é comum encontrar na literatura as regiões de estabilidade para alguns valores fixos de $K$ destes métodos, como em Meneguette (1987). Mesmo assim, a Figura 3.29 a seguir apresenta as regiões de estabilidade absoluta para alguns valores de $K$ para os métodos BDF.

No caso dos métodos $(K, L)$ para $L \geq 2$, já é mais difícil encontrar abordagens sobre a região de estabilidade absoluta. Portanto, a seguir é mostrado que este procedimento também pode ser 


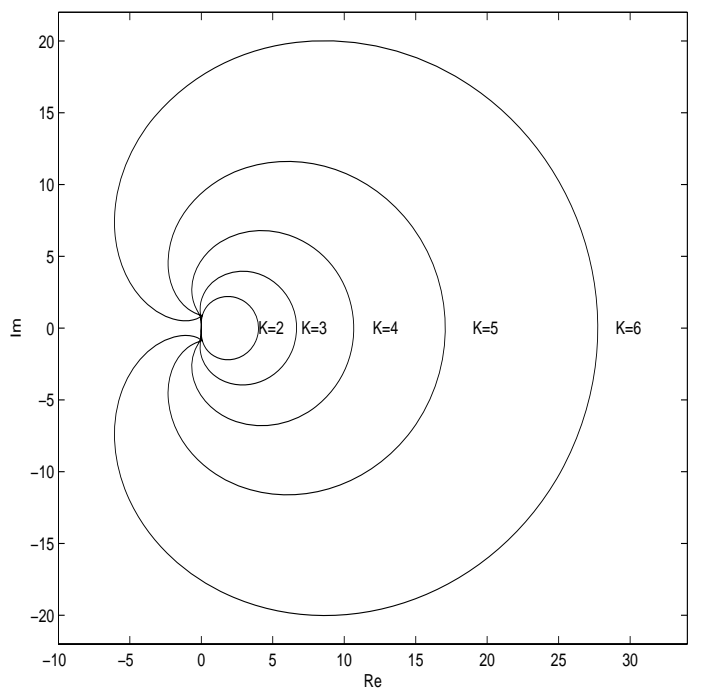

Figura 3.29: Regiões de estabilidade para os métodos de Brown para $L=1$ e $K=2, \ldots, 6$

utilizado para determinar as regiões de estabilidade dos métodos $(K, L)$ de Brown para $L \geq 2$, generalizando o que foi apresentado em Sofroniou (1996), pois agora são considerados métodos multiderivadas de passo múltiplo.

No caso dos métodos $(K, L)$ de Brown, o polinômio característico é dado por

$$
\pi(w, z)=\rho(w)-\sum_{j=1}^{L} z^{j} \sigma_{j}(w)
$$

onde $\rho(w)$ e $\sigma_{j}(w)$ são dados pelas expressões (3.1.1) e (3.1.2), respectivamente.

É possível representar as curvas soluções de $\pi(w, z)=0$, generalizando o que foi apresentado anteriormente, através da expressão

$$
\mathbf{w}(z)=\left[\begin{array}{c}
w_{1}(z) \\
w_{2}(z) \\
\vdots \\
w_{K}(z)
\end{array}\right] .
$$

Por exemplo, no caso do método $(2,2)$ de Brown, o polinômio $\pi(w, z)$ é dado por

$$
\pi(w, z)=\rho(w)-z \sigma_{1}(w)-z^{2} \sigma_{2}(w)
$$

onde

$$
\rho(z)=\frac{7}{4} w^{2}-2 w+\frac{1}{4}, \sigma_{1}(w)=\frac{3}{2} w^{2} \text { e } \sigma_{2}(w)=-\frac{1}{2} w^{2}
$$


As curvas soluções de $\pi(w, z)=0$ são dadas por

$$
\mathbf{w}(z)=\left[\begin{array}{c}
\frac{4+\sqrt{9+6 z-2 z^{2}}}{7-6 z+2 z^{2}} \\
\frac{4-\sqrt{9+6 z-2 z^{2}}}{7-6 z+2 z^{2}} .
\end{array}\right] .
$$

A seguir são apresentadas as curvas soluções do polinômio característico e as regiões de estabilidade de alguns métodos $(K, L)$ para alguns valores de $K$ e $L$ fixos, que ainda não foram publicadas na literatura.

A Figura 3.30 representa as curvas soluções do polinômio característico $\pi(w, z)=0$ no caso do método $(2,2)$ de Brown, cuja expressão é dada por (3.3.1).
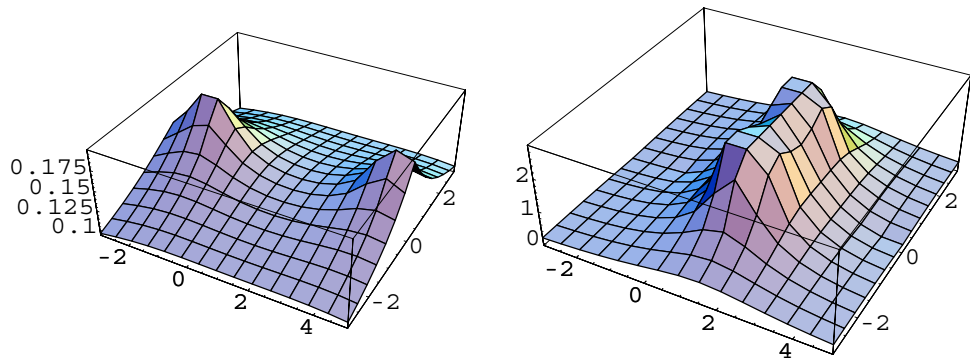

Figura 3.30: Curvas soluções do método $(2,2)$ de Brown

As regiões de estabilidade absoluta para os métodos $(2,2),(3,2)$ e $(4,2)$ de Brown podem ser visualizadas nas Figuras 3.31, 3.32 e 3.33, respectivamente. Convém lembrar que a região de estabilidade é a área externa à curva.

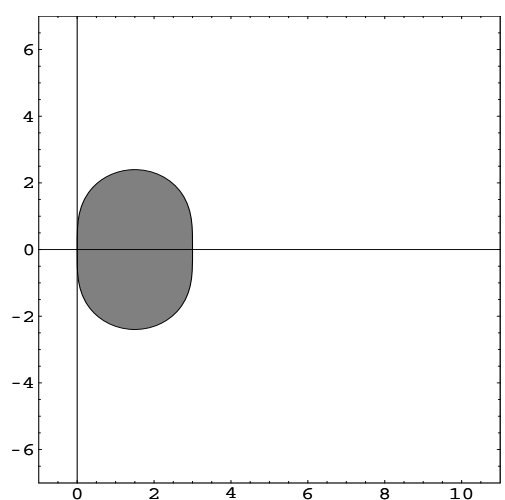

Figura 3.31: Região de estabilidade do método $(2,2)$ de Brown

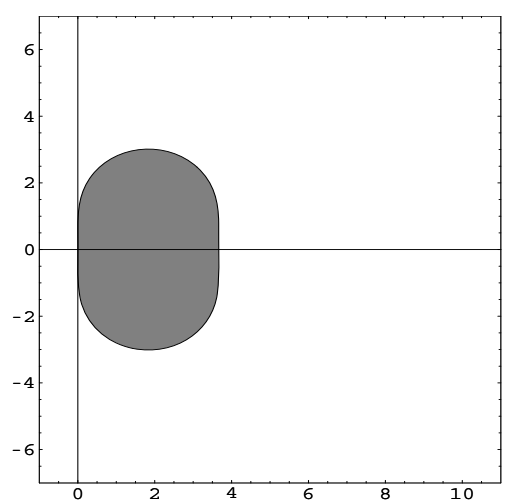

Figura 3.32: Região de estabilidade do método $(3,2)$ de Brown

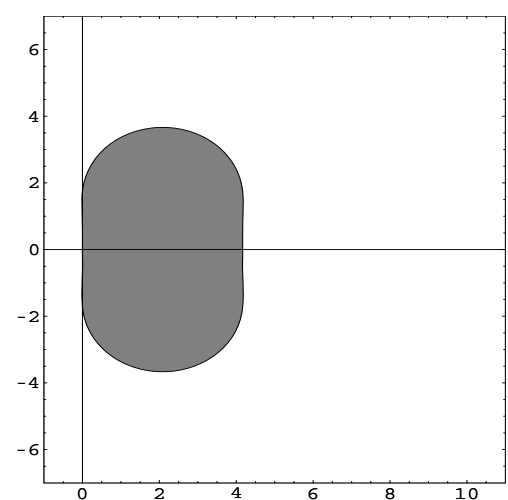

Figura 3.33: Região de estabilidade do método $(4,2)$ de Brown

As regiões de estabilidade absoluta para os métodos $(K, 2)$ de Brown para alguns valores fixos de $K$ também pode ser encontrada em Hairer e Wanner (1996), que descreve tais métodos pela 
denominação de "second derivative BDF methods". A Figura 3.34 representa tais regiões para $K=3, \ldots, 10$. Tais regiões são simétricas em relação ao eixo $x$.

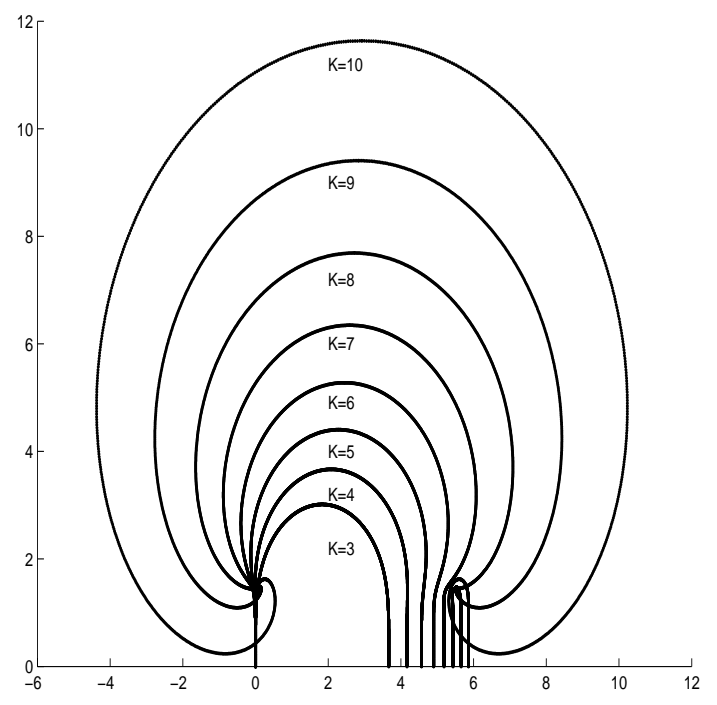

Figura 3.34: Regiões de estabilidade para os métodos de Brown para $L=2$ e $K=3, \ldots, 10$

É possível observar que no caso dos métodos $(K, 2)$, assim como nos métodos BDF, à medida que $K$ aumenta , diminui a região de estabilidade absoluta do método, e então, métodos com menor quantidade de passos apresentam maior região de estabilidade.

Quando $L=3$, por exemplo, no caso do método $(2,3)$ de Brown, as curvas soluções de $\pi(w, z)=0$ são dadas por

$$
\mathbf{w}(z)=\left[\begin{array}{c}
\frac{24+\sqrt{3} \sqrt{147+42 z-18 z^{2}+4 z^{3}}}{45-42 z+18 z^{2}-4 z^{3}} \\
\frac{-24+\sqrt{3} \sqrt{147+42 z-18 z^{2}+4 z^{3}}}{-45+42 z-18 z^{2}+4 z^{3}}
\end{array}\right],
$$

cuja representação gráfica pode ser observada na Figura 3.35.
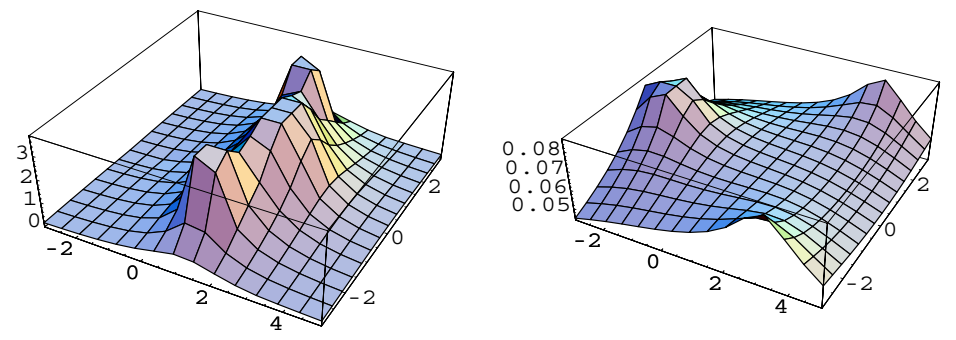

Figura 3.35: Curvas soluções do método $(2,3)$ de Brown

Nas Figuras 3.36, 3.37 e 3.38 é possível visualizar as regiões de estabilidade absoluta dos métodos $(2,3),(3,3)$ e $(4,3)$, respectivamente.

As regiões de estabilidade dos métodos $(2,4),(3,4)$ e $(4,4)$ podem ser visualizadas nas Figuras 3.39, 3.40 e 3.41, respectivamente. 


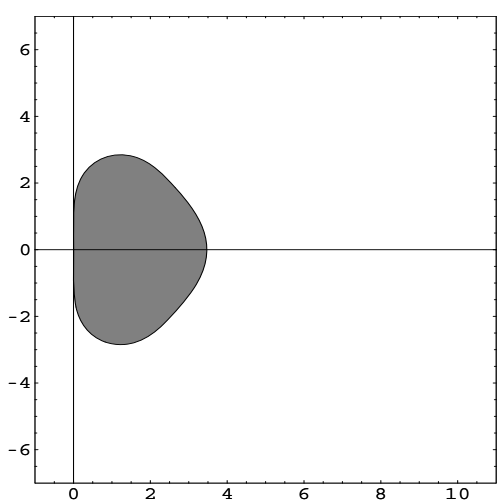

Figura 3.36: Região de estabilidade do método $(2,3)$ de Brown

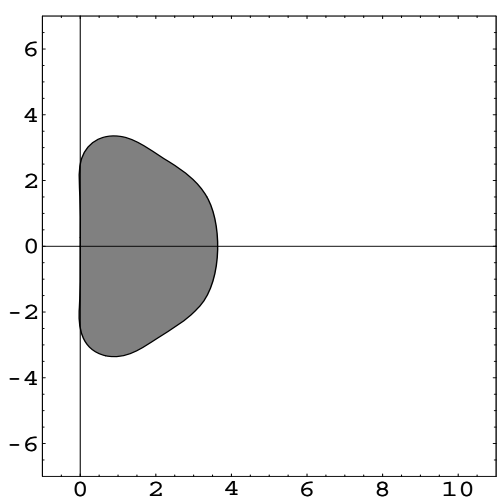

Figura 3.39: Região de estabilidade do método $(2,4)$ de Brown

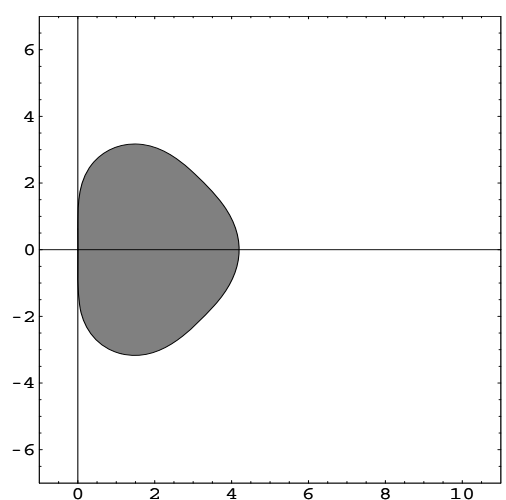

Figura 3.37: Região de estabilidade do método $(3,3)$ de Brown

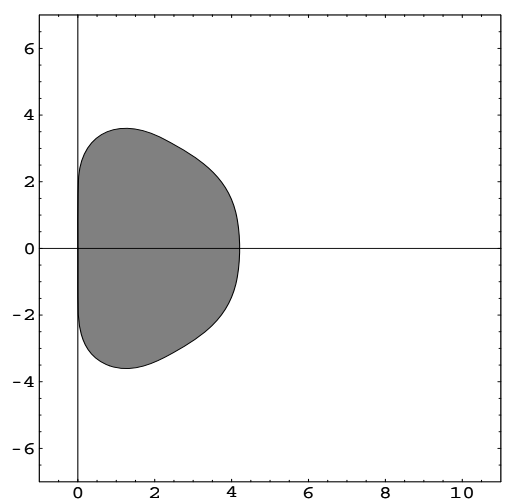

Figura 3.40: Região de estabilidade do método $(3,4)$ de Brown

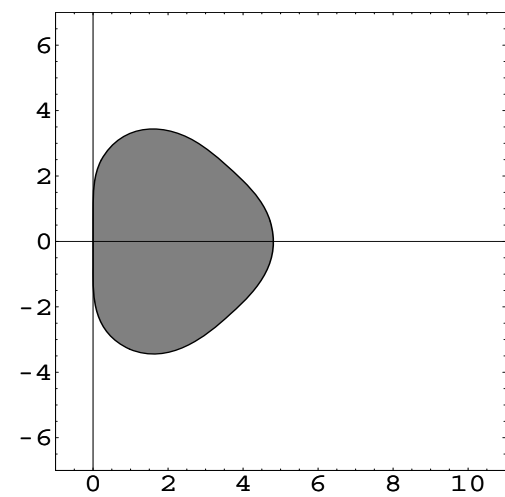

Figura 3.38: Região de estabilidade do método $(4,3)$ de Brown

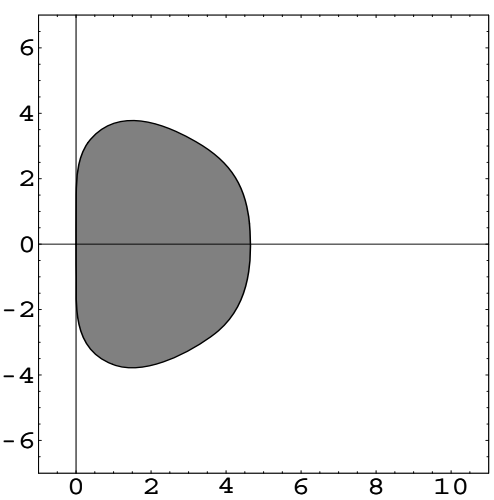

Figura 3.41: Região de estabilidade do método $(4,4)$ de Brown

Como já foi citado anteriormente, à medida que aumenta a quantidade de passos $(K)$, diminui a região de estabilidade absoluta do método $(K, L)$ de Brown, sendo esta uma característica que pode ser observada em todas as regiões de estabilidade apresentadas. 


\section{Considerações Finais}

Este capítulo tem o objetivo de mostrar os principais resultados obtidos neste trabalho e também apresentar alguns assuntos que ainda podem ser pesquisados, tanto na área de zeros de polinômios quanto na análise de métodos numéricos.

Muitas contribuições foram feitas na área de estabilidade dos métodos $(K, L)$ de Brown, com o uso das order stars, que ainda é uma ferramenta pouco utilizada, e muitos resultados obtidos através das order stars para os métodos $(K, L)$ também podem ser adaptados a outros métodos numéricos.

A seguir são relatados os resultados obtidos na área de zeros de polinômios e em seguida os resultados sobre os métodos $(K, L)$ de Brown são comentados.

\subsection{Sobre zeros de polinômios}

Em relação aos zeros de polinômios, a Conjectura 1.2 ainda é um resultado que precisa ser mais explorado. Existem fortes evidências de que ela é verdadeira e, como já foi visto no Capítulo 2, para alguns casos específicos, sua validade foi mostrada.

Por exemplo, no caso do polinômio reflexivo, foi mostrado, através da Proposição 2.2, que o polinômio $P_{\gamma}(z)$, sujeito às condições estabelecidas no resultado, tem todos os seus zeros no disco unitário. Portanto, no caso crítico em que os zeros do polinômio $P(z)$ encontram-se sobre o círculo unitário, a variação de $\gamma$ faz com que os zeros de $P_{\gamma}(z)$ entrem no disco unitário. Este resultado foi apresentado por Botta et al. (2007) no XXX Congresso Nacional de Matemática Aplicada e 
Computacional (CNMAC) e foi submetido à Revista TEMA (Tendências em Matemática Aplicada e Computacional) para publicação na Seleta do XXX CNMAC.

Além disso, foi mostrado que, para polinômios de grau menor ou igual a três, a Conjectura 1.2 é verdadeira. Para polinômios de grau maior que três, um dos problemas que surge é em relação ao sinal dos coeficientes de $P_{j, \gamma}^{*}(z), j=2, . . n-1$, como mencionado no Capítulo 2 . Se for possível mostrar que todos são positivos, não seria difícil provar o resultado, pois, neste caso, sabe-se que

$$
\left|a_{1, \gamma}^{(j)}\right|>\left|a_{2, \gamma}^{(j)}\right|>\ldots>\left|a_{n-j, \gamma}^{(j)}\right|
$$

para $j=1, \ldots, n-j, \mathrm{e}$

$$
\left|a_{0, \gamma}^{(n-1)}\right|>\left|a_{1, \gamma}^{(n-1)}\right|
$$

Assim, através do Teorema 2.5, a validade do resultado seria verificada.

Portanto, um desafio agora é mostrar que os coeficientes de $P_{j, \gamma}^{*}(z), j=2, . . n-1$, são todos positivos.

Muitos resultados foram utilizados na tentativa de mostrar a validade da Conjectura 1.2, mas, em algum momento, tais resultados se mostravam ineficientes ou inadequados. Por exemplo, através do critério de Schur-Cohn (Teorema 2.6), foi possível provar a conjectura para $n \leq 2$ mas, a partir de $n=3$, a ordem das matrizes obtidas dificulta o cálculo do determinante, que vem ilustrado a seguir.

Seja $P(z)=\sum_{i=0}^{n} a_{i} z^{i}$. Considere os determinantes $\Delta_{k}$ e $\Delta_{k, \gamma}, k=1,2, . ., n$, relacionados a $P(z)$ e $P_{\gamma}(z)$, respectivamente, cujas expressões são dadas no Teorema 2.6.

Para $k=1$,

$$
\Delta_{1, \gamma}-\Delta_{1}=-\gamma^{2}-2 a_{n} \gamma<0
$$

Logo,

$$
\Delta_{1, \gamma}<\Delta_{1} \leq 0
$$

Portanto, $\Delta_{1, \gamma}<0$.

Da mesma forma,

$\Delta_{2, \gamma}-\Delta_{2}=\gamma^{4}+4 a_{n} \gamma^{3}+\left(6 a_{n}^{2}-a_{1}^{2}-2 a_{0}^{2}\right) \gamma^{2}+\left(2 a_{0} a_{1} a_{n-1}-4 a_{0}^{2} a_{n}-2 a_{1}^{2} a_{n}+4 a_{n}^{3}\right) \gamma>0$

Então,

$$
\Delta_{2, \gamma}>\Delta_{2} \geq 0
$$

Logo, $\Delta_{2, \gamma}>0$. 
Para $k=3$,

$$
\begin{aligned}
\Delta_{3, \gamma}-\Delta_{3}= & -\gamma^{6}-6 a_{n} \gamma^{5}+\left(3 a_{0}^{2}+2 a_{1}^{2}+a_{2}^{2}-15 a_{n}^{2}\right) \gamma^{4}+ \\
+ & \left(-2 a_{0} a_{2} a_{n-2}-4 a_{0} a_{1} a_{n-1}-2 a_{1} a_{2} a_{n-1}+12 a_{0}^{2} a_{n}+8 a_{1}^{2} a_{n}+4 a_{2}^{2} a_{n}-20 a_{n}^{3}\right) \gamma^{3}+ \\
+ & \left(-3 a_{0}^{4}-2 a_{0}^{2} a_{1}^{2}-a_{1}^{4}+2 a_{0} a_{1}^{2} a_{2}-a_{0}^{2} a_{2}^{2}+a_{0}^{2} a_{n-2}^{2}+2 a_{0} a_{1} a_{n-2} a_{n-1}+2 a_{0}^{2} a_{n-1}^{2}+\right. \\
& +a_{1}^{2} a_{n-1}^{2}+2 a_{0} a_{2} a_{n-1}^{2}-6 a_{0} a_{2} a_{n-2} a_{n}-12 a_{0} a_{1} a_{n-1} a_{n}-6 a_{1} a_{2} a_{n-1} a_{n}+ \\
& \left.+18 a_{0}^{2} a_{n}^{2}+12 a_{1}^{2} a_{n}^{2}+6 a_{2}^{2} a_{n}^{2}-15 a_{n}^{4}\right) \gamma^{2}+ \\
+ & \left(-2 a_{0}^{2} a_{1}^{2} a_{n-2}+2 a_{0}^{3} a_{2} a_{n-2}+4 a_{0}^{3} a_{1} a_{n-1}+2 a_{0} a_{1}^{3} a_{n-1}-2 a_{0}^{2} a_{1} a_{2} a_{n-1}-\right. \\
& -2 a_{0}^{2} a_{n-2} a_{n-1}^{2}-2 a_{0} a_{1} a_{n-1}^{3}-6 a_{0}^{4} a_{n}-4 a_{0}^{2} a_{1}^{2} a_{n}-2 a_{1}^{4} a_{n}+4 a_{0} a_{1}^{2} a_{2} a_{n}- \\
& -2 a_{0}^{2} a_{2}^{2} a_{n}+2 a_{0}^{2} a_{n-2}^{2} a_{n}+4 a_{0} a_{1} a_{n-2} a_{n-1} a_{n}+4 a_{0}^{2} a_{n-1}^{2} a_{n}+2 a_{1}^{2} a_{n-1}^{2} a_{n}+ \\
& +4 a_{0} a_{2} a_{n-1}^{2} a_{n}-6 a_{0} a_{2} a_{n-2} a_{n}^{2}-12 a_{0} a_{1} a_{n-1} a_{n}^{2}-6 a_{1} a_{2} a_{n-1} a_{n}^{2}+12 a_{0}^{2} a_{n}^{3}+ \\
& \left.+8 a_{1}^{2} a_{n}^{3}+4 a_{2}^{2} a_{n}^{3}-6 a_{n}^{5}\right) \gamma .
\end{aligned}
$$

Neste caso, é preciso analisar os sinais dos coeficientes de $\gamma, \gamma^{2}$ e $\gamma^{3}$. Mas sem impor algumas condições adicionais sobre os coeficientes de $P(z)$ fica difícil analisá-los. Portanto, é possível concluir que o número de mudanças de sinal da seqüência $\left(1, \Delta_{1, \gamma}, \Delta_{2, \gamma}\right)$ é igual a dois e então segue que $P_{2, \gamma}(z)$ possui dois zeros no interior do disco unitário. Para $k=4$, será obtida uma expressão que é um polinômio de grau 8 na variável $\gamma$, o que torna muito complicado analisar o sinal de $\Delta_{4, \gamma}$. Logo, fica inviável utilizar o critério de Schur-Cohn na análise da Conjectura 1.2 para $n \geq 3$.

Portanto, o Capítulo 2 apresenta a análise da conjectura através da seqüência de polinômios $P_{j}^{*}(z), j=1, \ldots, n$, que foi o resultado que se mostrou mais adequado.

\subsection{Sobre os métodos $(K, L)$ de Brown}

Um dos principais objetivos deste trabalho foi o estudo da Conjectura 1.2, pois através dela foi possível mostrar que, para $K \leq K_{L}^{*}$, os métodos de Brown zero-estáveis são também $A_{0}$-estáveis. Então, os métodos $(K, L)$ são stiff-estáveis para $K \leq K_{L}^{*}$. Portanto, foi possível determinar uma grande subclasse de métodos stiff-estáveis, o que pode ser muito útil em estudos futuros, já que estes métodos são usados na solução de problemas stiff.

Além disso, outras contribuições deste trabalho foram a obtenção e demonstração de resultados já conhecidos utilizando order stars. Primeiramente foi generalizado o conceito de order stars para o caso dos métodos $(K, L)$ de Brown, pois na literatura os resultados se referem apenas aos métodos lineares. Portanto, como já foi visto anteriormente, as order stars para os métodos $(K, L)$ 
de Brown são geradas por

$$
\widetilde{\mu}(z)=\frac{\sum_{j=1}^{L} \sigma_{j}\left(e^{z}\right) z^{j-1}}{\rho\left(e^{z}\right)}-\frac{1}{z}, z \in C .
$$

Para se chegar a alguns resultados sobre a estabilidade dos métodos $(K, L)$ de Brown foi necessário apresentar as order stars para esta classe de métodos, sendo o caso $L=2$ apresentado em Botta e Meneguette (2006). Além disso, foram apresentadas as order stars para alguns valores fixos de $K$ e $L$ e através dessa ferramenta foi possível analisar algumas propriedades dos métodos $(K, L)$ de Brown. Por exemplo, foi mostrado que, para $K$ fixo e $L$ suficientemente grande, os métodos de Brown são zero-estáveis e para $L$ fixo e $K$ suficientemente grande, tais métodos são instáveis. As demonstrações destes resultados, que encontram-se em Jeltsch e Kratz (1978), são complicadas e requerem um grande número de lemas. Mas através das order stars tais resultados foram provados de maneira mais simples.

As order stars também foram utilizadas para mostrar que os zeros dos polinômios característicos associados aos métodos $(K, L)$ são distintos. Este resultado, no caso dos métodos BDF, foi publicado em Botta e Meneguette (2007).

Foram apresentadas também as regiões de estabilidade absoluta para alguns valores fixos de $K$ e $L$ dos métodos $(K, L)$, não publicados antes na literatura, através do software Mathematica e utilizando conceitos relacionados às order stars. 


\section{Referências Bibliográficas}

Anderson, N.; SAFF, E. B.; VARGA, R. S. On the Eneström-Kakeya theorem and its sharpness. Linear Algebra App., v. 28, p. 5-16, 1979.

Botta, V. A. Polinômios algébricos e trigonométricos com zeros reais. Dissertação de Mestrado, IBILCE/UNESP, São José do Rio Preto, 2003.

Botta, V. A.; Meneguette, M. Order stars para os métodos de Brown $(K, 2)$. TEMA, v. 7 , n. 1, p. 85-90, 2006.

Botta, V. A.; Meneguette, M. Zeros dos polinômios característicos dos métodos BDF. TEMA, v. 8, n. 1, p. 93-98, 2007.

Botta, V. A.; Meneguette, M.; Cuminato, J. A. Sobre uma generalização do teorema de Eneström-Kakeya: polinômio reflexivo. Submetido à Revista TEMA, 2007.

BROWN, R. L. Some characteristics of implicit multistep-multiderivative integration formulas. SIAM Journal Num. Anal., v. 14, p. 982-993, 1977.

HaIRER, E.; WANner, G. On the instability of the BDF formulas. SIAM Journal Num. Anal., v. 20, p. 1206-1209, 1983.

HAIRER, E.; WANNER, G. Solving ordinary differential equations II: stiff and differential algebraic problems. Springer-Verlag, New York, 1996.

Iserles, A.; Norsett, S. P. A proof of the first Dahlquist barrier by order stars. Bit, v. 24, p. $529-537,1984$.

Iserles, A.; Norsett, S. P. Order stars. Chapman and Hall, London, 1991.

JELTSCH, R. $\quad A_{0}$-stability and stiff stability of Brown's multistep multiderivative methods. $\mathrm{Nu}$ mer. Math., v. 32, p. 167-181, 1979. 
JELTSCH, R.; KRATZ, L. On the stability properties of Brown's multistep multiderivative methods. Numer. Math., v. 30, p. 25-38, 1978.

LAMBERT, J. D. Computational methods in ordinary differential equation. Wiley, New York, 1973.

MARDEn, M. The number of zeros of a polynomial in a circle. Proc. Nat. Acad. Sci. U.S.A., v. 34, p. $15-17,1948$.

Marden, M. Geometry of polynomials. Amer. Math. Soc., Providence, 1966.

Meneguette, M. Multistep multiderivative methods and related topics. Tese de Doutoramento, Linacre College, NAGroup-OUCL, Oxford University, 1987.

Meneguette, M. Zeros in the unit disk. SIAM Review, v. 36, p. 656-657, 1994.

Milovanóvic, G. V.; Mitrinovic, D. S.; Rassias, T. M. Topics in polynomials: Extremal problems, inequalities, zeros. World Scientific, Singapore, 1994.

Sofroniou, M. Order stars and linear stability theory. J. Symbolic Computation, v. 21, p. 101$131,1996$.

Wanner, G.; Hairer, E.; Norsett, S. P. Order stars and stability theorems. Bit, v. 18, p. $475-489,1978$. 